\title{
Necessary Conditions for Optimal Control of Diffusions with Hard Terminal State Restrictions
}

\author{
Atle Seierstad \\ Department of Economics, University of Oslo, Oslo, Norway \\ Email: atle.seierstad@econ.uio.no
}

How to cite this paper: Seierstad, A. (2018) Necessary Conditions for Optimal Control of Diffusions with Hard Terminal State Restrictions. Open Journal of Optimization, 7, 1-40.

https://doi.org/10.4236/ojop.2018.71001

Received: December 22, 2016

Accepted: March 19, 2018

Published: March 22, 2018

Copyright $\odot 2018$ by author and Scientific Research Publishing Inc. This work is licensed under the Creative Commons Attribution International License (CC BY 4.0).

http://creativecommons.org/licenses/by/4.0/

\begin{abstract}
Necessary conditions are proved for certain problems of optimal control of diffusions where hard end constraints occur. The main results apply to several dimensional problems, where some of the state equations involve Brownian motions, but not the equations corresponding to states being hard restricted at the terminal time. The necessary conditions are stated in terms of weak variations. Two versions of necessary conditions are given, one version involving solutions of variational equations, the other one involving first order adjoint equations.
\end{abstract}

\section{Keywords}

Optimal Control, Diffusions, Hard Terminal State Restrictions

\section{Introduction}

The purpose of the present paper is to prove necessary conditions for the optimal control of certain types of control problems involving diffusions where hard end constraints on solutions occur. The books [1] and [2] contain introductions to the topic of optimal control of diffusions. Various types of maximum principles have been proved for problems of control of diffusions in case of no or soft terminal state restrictions, see e.g. [3] [4] [5] and [6]. Maximum principles for problem with hard terminal restrictions are proved for certain types of continuous time controlled diffusion problems in [7]. In that paper only the drift term contained controls. In this paper controls are allowed to enter also the diffusion term. Singular controls are not discussed, below we merely consider problems where the controls appearing may be said to be absolutely continuous with respect to Lebesgue measure. The restriction to such 
controls makes it harder to operate with hard terminal state restrictions. In fact, the case discussed in detail in this paper is the case where hard terminal restrictions are only placed on states governed by differential equations not containing a Brownian motion. The necessary condititions are stated in terms of "weak" variations, not strong ones (needleshaped ones). Brownian motions will only appear in differential equations of states unconstrained at the terminal time. The constrained states are, however, influenced by other states directly influenced by Brownian motions, so, below, necessary conditions are stated and proved for such problems. Because the states are stochastic, the state space is infinite dimensional, so to obtain necessary conditions, one must impose a condition amounting to demanding sufficient variability of the first order variations in the problem.

\section{The Control Problem and the Statement of the Necessary Condition}

Let $T>0$ and let $x_{0}$ be a given point in the Euclidean space $\mathbb{R}^{n^{*}}$, let $\pi$ be a projection from $\mathbb{R}^{n^{*}}$ onto $\mathbb{R}^{m^{*}}, m^{*}<n^{*}$, such that $\pi x=\left(x_{1}, \cdots, x_{m^{*}}\right)$ $\left(x=\left(x_{1}, \cdots, x_{n^{*}}\right)\right)$ and let $U$ be a closed convex subset of a Euclidean space $\mathbb{R}^{m^{* *}}$. The symbol $|$.$| is used for the Euclidean norm in any Euclidean space \mathbb{R}^{k}$, including $\mathbb{R}^{1}$, and, applied to matrices, it is the linear operator norm. Furnish the interval $J:=[0, T]$ with the Lebesgue measure. Let $\left(\Omega, \Phi, \Phi_{t}, P\right)$ be a given filtered probability space, (i.e. for $t \in[0, T]$, the $\Phi_{t}$ 's are sub- $\sigma$-algebras of the given $\sigma$-algebra $\Phi$ of subsets of $\Omega, \Phi_{s} \subset \Phi_{t}$ if $s<t, P$ is a probability measure on $\Phi)$, and assume that $(\Omega, \Phi, P)$ is complete, that $\Phi_{0}$ contains all the $P$-null sets in $\Phi$ and that $\Phi_{t}$ is right continuous. Let $L_{p}\left(J \times \Omega, \mathbb{R}^{n}\right), \quad p \in[1, \infty)$, be the set of Lebesgue $\times \Phi$-measurable functions $z(t, \omega)$ for which $E \int_{J}|z(t, \omega)|^{p} \mathrm{~d} t<\infty$ and let $L_{\infty}\left(J \times \Omega, \mathbb{R}^{n}\right)$ be the subset of essentially bounded functions in $L_{1}\left(J \times \Omega, \mathbb{R}^{n}\right)$. Related to $\left(\Omega, \Phi, \Phi_{t}, P\right)$, let $B_{t}$ be a vector the $n^{\prime}$ components of which (denoted $B_{t}^{j}$ ) are independent one-dimensional Brownian motions all adapted to $\left\{\Phi_{t}\right\}_{t}$, such that $B^{j}(t)-B^{j}(s)$ is independent of $\Phi_{s}$ for all $s, t, 0 \leq s<t$, and is normally distributed with mean 0 and covariances $(t-s) I, I$ the identity matrix. In applications where the $B_{t}^{j}$ 's are entities that can be observed, it is natural to take $\left\{\Phi_{t}\right\}_{t}$ as the natural filtration generated by the $B_{t}^{j}$ s. There are given functions $f(t, x, u)$ and $\bar{\sigma}^{j}(t, x, u) \quad j=1, \cdots, n^{\prime}$, from $J \times \mathbb{R}^{n^{*}} \times \mathbb{R}^{n^{\prime *}}$ into $\mathbb{R}^{n^{*}}$. Let $U^{\prime}$ be the set of functions $u(t, \omega)$ taking values in $U$, such that $u(.,$.$) , for each t$, when restricted to $[0, t] \times \Omega$, is Borel $\times \Phi_{t}$-measurable (i.e. progressively measurable).

Let $|\cdot|_{2}$ be the $L_{2}$-norm both on $L_{2}\left(\Omega, \Phi, \mathbb{R}^{k}\right)$ and on $L_{2}\left(J \times \Omega, \Phi, \mathbb{R}^{k}\right)$.

The following conditions are called the Basic Assumptions.

$\mathbf{A}_{1}$ The functions $f(t, x, u)$ and $\bar{\sigma}^{j}(t, x, u)$ have derivatives $f_{x}, f_{u}$ and $\bar{\sigma}_{x}^{j}, \bar{\sigma}_{u}^{j}$ with respect to $x \in \mathbb{R}^{n^{*}}, u \in \mathbb{R}^{n^{* *}}$ that are continuous in $(x, u)$.

$\mathrm{A}_{2}$ As a function of $t$, the functions $f$ and $\bar{\sigma}^{j}$ and their derivatives have 
one-sided limits with respect to $t$.

Write $\bar{\sigma}$ for the $n^{*} \times n^{\prime}$-matrix whose columns are $\bar{\sigma}^{j}$, let $\bar{\sigma}_{x}^{j}\left(\bar{\sigma}_{u}^{j}\right)$ be the matrix with entries $\bar{\sigma}_{x_{i}}^{j}\left(\bar{\sigma}_{u_{i}}^{j}\right)$, and write $\sum_{j} \bar{\sigma}^{j} d B_{t}^{j}=\bar{\sigma} d B_{t}$. Also, write $1_{C}$ for the indicator function of the set $C$.

The following assumptions are called the Global Assumptions.

$\mathbf{B}_{1} \pi f_{x}$ and $\pi f_{u}$ are uniformly continuous in $x, u$, uniformly in $t$.

$\mathbf{B}_{2}$ For some constant $M$, for some given $x_{0}$ in $\mathbb{R}^{n^{*}}$, for all $u \in U$,

$$
\sup _{t}\left|f\left(t, x_{0}, u\right)\right| \leq M(1+|u|), \sup _{j, t}\left|\bar{\sigma}^{j}\left(t, x_{0}, u\right)\right| \leq M(1+|u|) .
$$

$\mathbf{B}_{3}$ A constant $M^{+}$exists such that for all $(t, x, u) \in J \times \mathbb{R}^{n^{*}} \times U$,

$$
\left|f_{x}(t, x, u)\right| \leq M^{+},\left|f_{u}(t, x, u)\right| \leq M^{+},\left|\bar{\sigma}_{x}^{j}(t, x, u)\right| \leq M^{+},\left|\bar{\sigma}_{u}^{j}(t, x, u)\right| \leq M^{+} \forall j
$$

$\mathrm{B}_{4}$

$$
\pi \bar{\sigma}^{j}=0 \text { for all } j \text {. }
$$

Let $L_{p}^{\text {prog }}\left(J \times \Omega, \mathbb{R}^{n}\right)$ be the set of progressively measurable functions in $L_{p}\left(J \times \Omega, \Phi, \mathbb{R}^{n}\right), \quad p \in[1, \infty]$. From now on we require that all control functions $u(.,$.$) belong to U^{\prime} \cap L_{2}^{\text {prog }}\left(J \times \Omega, \mathbb{R}^{n^{* *}}\right)$. The strong (unique) solution-continuous in $t$-of the equation

$$
x(t, \omega)=x_{0}+\int_{0}^{t} f(s, x(s, \omega), u(s, \omega)) \mathrm{d} s+\int_{0}^{t} \bar{\sigma}(s, x(s, \omega), u(s, \omega)) \mathrm{d} B_{s}
$$

is denoted $x^{u(.,)}(t, \omega)=x^{u}(t, \omega)$.

Let $a \in \mathbb{R}^{n^{*}}$ ( $a$ fixed, $\neq 0$ ) such that $\pi a=0$, let $\langle.,$.$\rangle denote scalar product,$ and consider the problem

$$
\max _{u(. .) \in U^{\prime} \cap L_{2}^{p r o g}\left(J \times \Omega, \mathbb{R}^{n^{* *}}\right)} E\left\langle x^{u(. .)}(T, .), a\right\rangle,
$$

subject to

$$
\pi x^{u(. .)}(T, \omega)=\tilde{y} \text { a.s., where } \tilde{y} \text { is fixed in } \mathbb{R}^{m^{*}} \text {. }
$$

Below, $u^{*}(.,.) \in U^{\prime} \cap L_{2}^{\text {prog }}\left(J \times \Omega, \Phi, \mathbb{R}^{n^{* *}}\right)$ will be an optimal control in this problem, assumed to exist. Write $x^{u^{*}(. .)}(.,)=.x^{*}(.,$.$) .$

We have collected a few definitions that are going to be used in the sequel.

For $z(.,.) \in L_{2}\left(J \times \Omega, \Phi, \mathbb{R}^{n}\right)$, let

$$
\begin{gathered}
\|z(., .)\|_{2}:=\operatorname{essup}_{t}\left(E\left[|z(t, \omega)|^{2}\right]\right)^{1 / 2},\|z(., .)\|_{2}^{*}:=\left(E\left[\left(\operatorname{essup}_{t}|z(t, \omega)|\right)^{2}\right]\right)^{1 / 2}, \\
\mathrm{cl}_{2}: \text { closure in } L_{2}^{\text {prog }}\left(J \times \Omega, \mathbb{R}^{n}\right) \text { in the norm }\|\cdot\|_{2}, \\
L_{2}^{n}:=\left\{z(., .) \in L_{2}^{\text {prog }}\left(J \times \Omega, \mathbb{R}^{n}\right):\|z(., .)\|_{2}<\infty\right\}, \\
B_{\alpha}^{n}:=\left\{z(., .) \in L_{2}^{n}:\|z(., .)\|_{2}<\alpha\right\}, \\
L_{2}^{* n}:=\operatorname{cl}_{2}\left\{z(., .) \in L_{2}^{\text {prog }}\left(J \times \Omega, \mathbb{R}^{n}\right):\|z(., .)\|_{2}^{*}<\infty\right\}, \\
B_{\alpha}^{* n}:=\left\{z(., .) \in L_{2}^{* n}:\|z(., .)\|_{2}<\alpha\right\},
\end{gathered}
$$

where $\alpha \in(0, \infty)$. Let 


$$
L^{2}=\left\{\int_{0}^{T} z(s ., .) \mathrm{d} s: z(., .) \in L_{2}^{m^{*}}\right\}, B^{\alpha}:=\left\{\int_{0}^{T} Z(s ., .) \mathrm{d} s: z(., .) \in B_{\alpha}^{m^{*}}\right\} .
$$

Finally, let

$$
\begin{aligned}
U^{*}: & =\left\{\hat{w}(., .) \in L_{2}^{* n^{* *}}: u^{*}(t, \omega)+\hat{w}(t, \omega) \in U \forall(t, \omega),\|\hat{w}(., .)\|_{2} \leq 1\right\}, \\
U^{* *}: & =u^{*}+U^{*}, \\
& U_{*}:=\left\{u(., .) \in L_{2}^{\text {prog }}\left(J \times \Omega, \mathbb{R}^{n^{* *}}\right): u(t, \omega) \in U \forall(t, \omega)\right\} .
\end{aligned}
$$

In the subsequent necessary conditions, the following local linear controllability condition (15) is needed. There exist numbers $\delta_{*}>0, \alpha>0$ and $c \in[0, T)$, and a progressively measurable function $\breve{Z}(t, \omega) \in L_{2}^{* *^{*}}$ such that for all $u_{*} \in U_{*} \cap L_{2}^{* n^{* *}}$ for which

$$
\begin{aligned}
\operatorname{essup}_{t}\left|u_{*}(t, .)-u^{*}(t, .)\right|_{2}:=\left\|u_{*}(., .)-u^{*}(., .)\right\|_{2} \leq \delta_{*}, \\
1_{[c, T]}\left[\breve{z}(., .)+B_{\alpha}^{* m^{*}}\right] \subset \Gamma:=\operatorname{cl}_{2}\left\{1_{[c, T]} \pi f_{u}\left(., x^{u_{*}}(., .), u_{*}(., .)\right) w(., .): w(., .) \in U^{*}\right\} .
\end{aligned}
$$

A more "concrete" condition implying (15) is presented in Remark 3.

Theorem 1. Assume that $u^{*}(.,$.$) is optimal in problem (5),(6), that$ Assumptions A and B hold (the Basic and Global Assumptions) and that (15) is satisfied. Then the following necessary conditions hold: For some linear functional $v$ on $L^{2}$, bounded on $B^{1}$, and some number $\Lambda_{0} \geq 0$, for all $w(.,.) \in U^{*}$,

$$
\left\langle\pi q^{w}(T, .), v\right\rangle+\Lambda_{0} E\left[\left\langle q^{w}(T, .), a\right\rangle\right] \leq 0,
$$

where $q^{w}(t, \omega)$ is the solution of

$$
\begin{aligned}
\mathrm{d} q^{w}(t, \omega)= & f_{x}\left(t, x^{*}(t, \omega), u^{*}(t, \omega)\right) q^{w}(t, \omega) \mathrm{d} t \\
& +f_{u}\left(t, x^{*}(t, \omega), u^{*}(t, \omega)\right) w(t, \omega) \mathrm{d} t \\
& +\sum_{j}\left[\bar{\sigma}_{x}^{j}\left(t, x^{*}(t, \omega), u^{*}(t, \omega)\right) q^{w}(t, \omega)\right. \\
& \left.+\bar{\sigma}_{u}^{j}\left(t, x^{*}(t, \omega), u^{*}(t, \omega)\right) w(t, \omega)\right] \mathrm{d} B_{t}^{j} .
\end{aligned}
$$

with $q^{w}(0, \omega)=0$. Moreover ${ }^{1},\left(\Lambda_{0},\left.v\right|_{L_{2}^{*^{*} m^{*}}}\right) \neq 0$.

Remark 1. If (15) holds for $\breve{Z}(. .)=$.0 , then $\Lambda_{0} \neq 0$. Moreover, when $\left\|u^{*}\right\|_{2}^{*}<\infty$, (21) below holds and $t \rightarrow \Phi_{t}$ is (also) left continuous, then both $\Lambda_{0} \neq 0$ and $v$ is a continuous linear functional on $L_{2}\left(\Omega, \Phi_{T}, \mathbb{R}^{m^{*}}\right)$ then holds for $\breve{Z}(.,)=$.0 ).

Remark 2. Let $s<t \leq T$, and let $C(t, s, \omega)$ be the resolvent of the equation

$$
\begin{aligned}
\breve{q}(t, \omega)= & \breve{q}_{s}(\omega)+\int_{s}^{t} f_{x}\left(s, x^{*}(s, \omega), u^{*}(s, \omega)\right) \breve{q}(s, \omega) \mathrm{d} t \\
& +\sum_{j} \int_{s}^{t} \bar{\sigma}_{x}^{j}\left(s, x^{*}(s), u^{*}(s, \omega)\right) \breve{q}(s, \omega) \mathrm{d} B_{s}^{j},
\end{aligned}
$$

$\breve{q}_{s}(.) \in L_{2}\left(\Omega, \Phi_{s}, \mathbb{R}^{n^{*}}\right)$, so $C(s, s, \omega)=I, \quad I$ the identity map in $\mathbb{R}^{n^{*}}$, and

${ }^{1} \mid L_{2}^{*}{ }^{*}$ means restriction to $L_{2}^{m m^{*}}$. 
$\breve{q}(t,)=.C(t, s,.) \breve{q}_{s}($.$) . Let v_{*}:=\phi \rightarrow\langle\pi \phi, v\rangle+\Lambda_{0} E\langle\phi, a\rangle$, $\phi \in\left\{\phi \in L_{2}\left(\Omega, \Phi_{T}, \mathbb{R}^{n^{*}}\right): \pi \phi \in L^{2}\right\}$. For $t<T, \psi(.) \rightarrow\left\langle C(T, t,.) \psi(),. v_{*}\right\rangle$, $\psi \in L_{2}\left(\Omega, \Phi_{t}, \mathbb{R}^{n^{*}}\right)$, is continuous in $|\cdot|_{2}$-norm and hence can be represented by $\quad$ an $\quad L_{2}$-function $\quad p^{-}(t,.) \in L_{2}\left(\Omega, \Phi_{t}, \mathbb{R}^{n^{*}}\right), \quad p^{-}(t, \omega)$ progressively measurable and right continuous in $t$, a.s. and in $|\cdot|_{2}$ (even continuous in this manner if $t \rightarrow \Phi_{t}$ is continuous). Note that if $w=0$ on $[s, T]$ then (16) implies $0 \geq\left\langle q^{w}(T,),. v_{*}\right\rangle=\left\langle C(T, s,.) q^{w}(s,),. v_{*}\right\rangle=E\left\langle q^{w}(s,),. p^{-}(s,).\right\rangle$.

Let $\Phi_{t}$ be the natural filtration generated by the $B_{t}^{j}$ 's, $\Phi_{0}$ augmented by the $P$-null sets in $\Phi$. The function $p^{-}(t, \omega)$, now taken to be a row vector, then satisfies the following condition: On $[0, T)$, there exist $\mathbb{R}^{n^{*}}$-valued, progressively measurable functions $p(t, \omega), q^{j}(t, \omega), j=1, \cdots, n^{\prime}$, all row vectors, $p(t, \omega)$ continuous in $t$, such that $E \int_{0}^{t}|p(t, .)|^{2} \mathrm{~d} t<\infty$, $E \int_{0}^{t}\left|q^{j}(t, .)\right|^{2} \mathrm{~d} t<\infty$ for all $t<T$, and such that

$$
\begin{aligned}
\mathrm{d} p(t, \omega)= & -p(t, \omega) f_{x}\left(t, x^{*}(t, \omega), u^{*}(t, \omega)\right) \mathrm{d} t \\
& -\sum_{j} q^{j}(t, \omega) \bar{\sigma}_{x}^{j}\left(t, x^{*}(t, \omega), u^{*}(t, \omega)\right) \mathrm{d} t+\sum_{j} q^{j}(t, \omega) \mathrm{d} B_{t}^{j}
\end{aligned}
$$

and such that, for all $t<T, \quad p^{-}(t, w)=p(t, \omega)$ a.s.

In this case, we have that for all $u \in U$, for a.e. $t$, a.s.

$$
\left\{p(t, .) f_{u}\left(t, x^{*}(t, .), u^{*}(t, .)\right)+\sum_{j} q^{j}(t, .) \bar{\sigma}_{u}^{j}\left(t, x^{*}(t, .), u^{*}(t, .)\right)\right\}\left(u-u^{*}(t, .)\right) \leq 0 .
$$

When $\left\|u^{*}\right\|_{2}^{*}<\infty, t \rightarrow \Phi_{t}$ is left continuous, and (21) below holds, the following additional properties hold: $E \int_{0}^{T}|p(t, .)|^{2} \mathrm{~d} t<\infty, E \int_{0}^{T}\left|q^{j}(t, .)\right|^{2} \mathrm{~d} t<\infty$, the $|\cdot|_{2}$-limit (and a.s. $\operatorname{limit}_{\text {) }} \lim _{t \rightarrow T} p(t,$.$) exists,$

$\operatorname{Pr}\left[\left(\Lambda_{0}, \lim _{t \rightarrow T} p(t, \omega)\right) \neq 0\right]>0$, and finally

$$
\text { for each component } j>m^{*}, \lim _{t \rightarrow T} p(t, .)_{j}=\Lambda_{0} a_{j} \text {. }
$$

Remark 3 The condition (15), with $\breve{Z}()=$.0 , is implied by the following conditions: $\left\|u^{*}\right\|_{2}^{*}<\infty$ and for some $\beta_{0}>0$, for all $(t, x, u)$,

$$
\text { for any } y \in \mathbb{R}^{m^{*}} \text {, for some } u^{\prime} \in U, y=\pi f_{u}(t, x, u) u^{\prime},\left|u^{\prime}\right| \leq \beta_{0}|y| \text {. }
$$

The $|\cdot|_{2}$-norm (or the norm $\|\cdot\|_{2}$ ) used in the formulation of the condition (15) can have quite strong consequences. Assume for example that $m^{*}=n^{* *}$, and, for some $\alpha>0$, that $\left|\operatorname{det}\left(\pi f_{u}\right)\right|>\alpha>0$ for all $(t, x, u)$. Then for some $\beta^{*}>0, \quad B\left(0, \beta^{*}\right) \subset \pi f_{u}(t, x, u)[\tilde{U}]$ for all $(t, x, u)$, if $\tilde{U}=B(0,1)$ and $U=\mathbb{R}^{n^{* *}}\left(B\left(0, \beta^{*}\right)\right.$ and $\tilde{U}$ balls in $L_{2}\left(\Omega, \Phi_{t},.\right)$-spaces, $\left.t<T\right)$, but if $U$ is strictly smaller than $\mathbb{R}^{n^{* * *}}$ it is neither possible to obtain this inclusion nor (15). When $n^{* *}>m^{*}$ the situation may be different. Consider in particular the case where the original terminal constraint is, say $x_{i}(T) \geq \tilde{y}_{i}, i=1, \cdots, m^{*}$. Using 
auxiliary controls $v_{i}$ and auxiliary states $z$ and $y$, the system can be rewritten as a system where the end constraints are $z_{i}(T) \geq \tilde{y}_{i}, \quad i=1, \cdots, m^{*}$, by adding new state equations $\dot{y}_{i}=v_{i}, v_{i} \geq 0, \quad i=1, \cdots, m^{*}, y_{i}(0)=0$, and by changing the first $m^{*}$ state equations to $\operatorname{read} \dot{z}=\pi f(t, z+y, u)-v=\hat{f}(t, z, y, u, v)$, $v=\left(v_{1}, \cdots, v_{m^{*}}\right), \quad z(0)=x_{0}$. Define $S_{\beta}^{n}:=\left\{x \in \mathbb{R}^{n}: x^{i} \in[0, \beta)\right\}$. We assume now $U=S_{\infty}^{n^{* *}}$, so the control set in the rewritten system is now $\breve{U}=S_{\infty}^{n^{* *}} \times S_{\infty}^{m^{*}}$. The control $\left(u^{*}(t, \omega), 0\right)$ is optimal in the rewritten system. Assume that $S_{\beta^{*}}^{m^{*}} \subset \pi f_{u}(t, x, u) S_{1}^{n^{* *}}-S_{\infty}^{m^{*}}$ for some $\beta^{*}>0$ for all $(t, x, u)$. Then, for some $\beta_{*}$, for all $(t, x, u), \operatorname{cl} B\left(0, \beta_{*}\right) \subset \hat{f}_{(u, v)}(t, x, u)[\tilde{U}]$, for $\tilde{U}=\left\{\left(u^{\prime}, v\right) \in R^{n^{* *}} \times R^{m^{*}}: u_{i}^{\prime} \geq 0, v_{i} \geq 0,\left|\left(u^{\prime}, v\right)\right| \leq 1\right\}, B\left(0, \beta_{*}\right)$ a ball in $\mathbb{R}^{m^{*}}$. Then for all $(t, x, u), \operatorname{cl} B\left(0, \alpha \beta_{*}\right) \subset \hat{f}_{(u, v)}(t, x, u)[\alpha U]$ for all $\alpha \geq 0$ so for all $(t, x, u)$,

$B_{2}^{t}\left(0, \beta_{*}\right) \cap L_{\infty}\left(\Omega, \Phi_{t}, R^{m^{*}}\right) \subset\left\{\hat{f}_{(u, v)}(t, x, u)\left[\left(u^{\prime}, v\right)\right]:\left(u^{\prime}, v\right) \in L_{\infty}\left(\Omega, \Phi_{t}, R^{n^{* *}}\right)\right.$

$\left.\times L_{\infty}\left(\Omega, \Phi_{t}, R^{m^{*}}\right): u_{i} \geq 0, v_{i} \geq 0,|(u, v)|_{2} \leq 1\right\}$

$B_{2}^{t}\left(0, \beta_{*}\right)$ a ball in $L_{2}\left(\Omega, \Phi_{t}, R^{m^{*}}\right)$. Hence, for all $u_{*}$,

$B_{\beta_{*}}^{* m^{*}} \subset \mathrm{cl}_{2}\left\{\hat{f}_{(u, v)}\left(., x^{u_{*}}, u_{*}\right)[(u, v)]:(u, v) \in \bar{U}\right\}$, where

$\bar{U}=\left\{(u, v) \in L_{2}^{* n^{* *}} \times L_{2}^{* m^{*}}: u_{i} \geq 0, v_{i} \geq 0,\|(u, v)\|_{2} \leq 1\right\} \quad$ (Necessary conditions for

systems with inequality constraints are stated in Remark 5 below, the local linear controllability condition just obtained suffices for the controllability condition in Remark 5 to apply to the original system).

\section{Proof of Theorem 1}

The proof consists of three lemmas and six proof steps A.-F., and relies on an "abstract" maximum principle, Corollary I in Appendix.

Without loss of generality, from now on let $x_{0}=0, T=1$ and let $J=[0,1]$. Note that $\|x(., .)\|_{2} \leq\|x(., .)\|_{2}^{*}$. For any $u(.,),. u^{\prime}(.,) \in U_{*}$, let $q^{u^{\prime}, u}(t, \omega)$ be the solution of

$$
\begin{aligned}
q^{u^{\prime}, u}(t, \omega)= & \int_{0}^{t} f_{x}\left(s, x^{u}(s, \omega), u(s, \omega)\right) q^{u^{\prime}, u}(s, \omega) \mathrm{d} s \\
& +\sum_{j} \int_{0}^{t} \bar{\sigma}_{x}^{j}\left(s, x^{u}(s, \omega), u(s, \omega)\right) q^{u^{\prime}, u}(s, \omega) \mathrm{d} B_{s}^{j} \\
& +\int_{0}^{t} f_{u}\left(s, x^{u}(s, \omega), u(s, \omega)\right)\left[u^{\prime}(s, \omega)-u(s, \omega)\right] \mathrm{d} s \\
& +\sum_{j} \int_{0}^{t} \bar{\sigma}_{u}^{j}\left(s, x^{u}(s, \omega), u(s, \omega)\right)\left[u^{\prime}(s, \omega)-u(s, \omega)\right] \mathrm{d} B_{s}^{j} .
\end{aligned}
$$

By general existence results and (1) and (2), $q^{u^{\prime}, u}(.,$.$) , and x^{u}(.,$.$) , see (4), do$ exist, both unique, strong solutions, continuous in $t$ (both column vectors).

A) Growth properties.

When $u(.,.) \in U_{*}$, by (1),

$$
\begin{aligned}
& E \int_{0}^{t}|f(s, 0, u(s, \omega))|^{2} \mathrm{~d} s \leq E \int_{0}^{t} M^{2}(1+|u(s, \omega)|)^{2} \mathrm{~d} s \\
& \leq E \int_{0}^{t} 2 M^{2}\left(1+|u(s, \omega)|^{2}\right) \mathrm{d} s \leq 2 M^{2}\left(1+\left(|u(., .)|_{2}\right)^{2}\right)
\end{aligned}
$$


and, similarly, $E \int_{0}^{t}\left|\bar{\sigma}^{j}(s, 0, u(s, \omega))\right|^{2} \mathrm{~d} s \leq 2 M^{2}\left(1+\left(|u(., .)|_{2}\right)^{2}\right)$. By (2), (4), and (57) in Appendix, Lemma A, (a consequence of Gronwall's inequality), with $\breve{z}(t, \omega)=x^{u}(t, \omega), \breve{h}=f(t, x, u(t, \omega)), \quad \sigma_{*}^{j}=\bar{\sigma}^{j}(t, x, u(t, \omega)), \quad \breve{z}_{0}=0, \quad \breve{y} \equiv 0$, $\beta(t, \omega)=-f(s, 0, u(s, \omega)), \quad \beta_{j}^{*}(t, \omega)=-\bar{\sigma}^{j}(t, 0, u(t, \omega)), \quad a^{*}(t, \omega)=\alpha(t, \omega)=0$, we have, for some constant $\breve{D}^{*}$ independent of $u(.,.) \in U_{*}$ (only dependent on $\mathrm{M}^{+}$), that

$$
\left\|x^{u}(., .)\right\|_{2}^{*} \leq \breve{D}^{*}\left(1+n^{\prime}\right) 2 M\left[1+|u(., .)|_{2}\right] .
$$

Let $u^{\prime \prime}, u \in U_{*}$. Using (2), and (57) in Appendix, Lemma A, (with $\breve{z}=x^{u}$, $\breve{y}=x^{u^{\prime \prime}}, \quad \beta^{*}=\beta=0, \breve{h}=f\left(t, x, u^{\prime \prime}(t, \omega)\right), \quad \sigma_{*}=\bar{\sigma}, \quad \breve{y}_{0}=\breve{z}_{0}=0$, $\alpha=f\left(s, x^{u}(s, \omega), u(s, \omega)\right)-f\left(s, x^{u}(s, \omega), u^{\prime \prime}(s, \omega)\right)$, $\alpha^{*}=\bar{\sigma}\left(s, x^{u}(s, \omega), u(s, \omega)\right)-\bar{\sigma}\left(s, x^{u}(s, \omega), u^{\prime \prime}(s, \omega)\right)$, for some constant $D$ independent of $u^{\prime \prime}$ and $u$ (only dependent on $M^{+}$), we get

$$
\begin{aligned}
& \left\|x^{u^{\prime \prime}}(., .)-x^{u}(. . .)\right\|_{2}^{*} \\
& \leq D\left(E \int_{0}^{1}\left|f\left(s, x^{u}(s, \omega), u(s, \omega)\right)-f\left(s, x^{u}(s, \omega), u^{\prime \prime}(s, \omega)\right)\right|^{2} \mathrm{~d} s\right)^{1 / 2} \\
& \quad+D \sum_{j}\left(E \int_{0}^{1}\left|\bar{\sigma}^{j}\left(s, x^{u}(s, \omega), u(s, \omega)\right)-\bar{\sigma}^{j}\left(s, x^{u}(s, \omega), u^{\prime \prime}(s, \omega)\right)\right|^{2} \mathrm{~d} s\right)^{1 / 2} \\
& \leq D M^{+}\left(1+n^{\prime}\right)\left|u^{\prime \prime}-u\right|_{2},
\end{aligned}
$$

(the last inequality by (2)).

Let $u^{\prime}, u \in U_{*}$. As explained below, for some $\breve{D}^{* *}>0$, we have

$$
\begin{aligned}
& \mid\left(\sup _{s \leq t}\left|q^{u^{\prime}, u}(s, .)\right|||_{2}\right. \\
& \leq \breve{D}^{* * *}\left(E \int_{0}^{t}\left|f_{u}\left(\breve{s}, x^{u}(\breve{s}, \omega), u(\breve{s}, \omega)\right)\left(u^{\prime}(\breve{s}, \omega)-u(\breve{s}, \omega)\right)\right|^{2} \mathrm{~d} \breve{s}\right)^{1 / 2} \\
& +\breve{D}^{* *} \sum_{j}\left(E \int_{0}^{t}\left|\bar{\sigma}_{u}^{j}\left(\breve{s}, x^{u}(\breve{s}, \omega), u(\breve{s}, \omega)\right)\left(u^{\prime}(\breve{s}, \omega)-u(\breve{s}, \omega)\right)\right|^{2} \mathrm{~d} \breve{s}\right)^{1 / 2} \\
& \leq \breve{D}^{* *}\left(1+n^{\prime}\right) M^{+}\left(E \int_{0}^{t}\left|u^{\prime}(\breve{s}, \omega)-u(\breve{s}, \omega)\right|^{2} \mathrm{~d} \breve{s}\right)^{1 / 2} \\
& \leq \breve{D}^{* *}\left(1+n^{\prime}\right) M^{+}\left|u^{\prime}(., .)-u(., .)\right|_{2} .
\end{aligned}
$$

The inequality (25) follows from (57) in Appendix, Lemma A, together with (2), for $\breve{z}=q^{u^{\prime}, u}, \breve{h}(t, x, \omega)=f_{x}\left(t, x^{u}(t, \omega), u(t, \omega)\right)[x]$,

$\sigma_{*}^{j}(t, x, \omega)=\bar{\sigma}_{x}^{j}\left(t, x^{u}(t, \omega), u(t, \omega)\right)[x], \quad \breve{y}=\beta=\beta^{*} \equiv 0 \quad\left(\breve{z}_{0}=\breve{y}_{0}=0\right)$, and

$$
\begin{aligned}
\alpha(t, \omega) & :=f_{u}\left(t, x^{u}(t, \omega), u(t, \omega)\right)\left(u^{\prime}(t, \omega)-u(t, \omega)\right) \\
\alpha_{j}^{*}(t, \omega) & :=\bar{\sigma}_{u}^{j}\left(t, x^{u}(t, \omega), u(t, \omega)\right)\left(u^{\prime}(t, \omega)-u(t, \omega)\right)
\end{aligned}
$$

Similarly ${ }^{2}$, for $u^{\prime \prime}, u^{\prime}, u \in U_{*}$,

${ }^{2}$ Lemma A applies for $\breve{h}(t, x, \omega)=f_{x}\left(t, x^{u}(t), u(t)\right)[x]+f_{u}\left(t, x^{u}(t), u(t)\right)\left(u^{\prime}(t)-u(t)\right)$,

$\sigma_{\cdot}^{j}(t, x, \omega)=\bar{\sigma}_{x}^{j}\left(t, x^{u}(t, \omega), u(t, \omega)\right)[x]+\bar{\sigma}_{u}^{j}\left(t, x^{u}(t, \omega), u(t, \omega)\right)\left(u^{\prime}(t)-u(t)\right), \quad \alpha=0, \quad \alpha^{*}=0$,

$\breve{z}=q^{u, u}, \quad \breve{y}=q^{u, u}, \quad \beta=f_{u}\left(t, x^{u}(t), u(t)\right)\left(u^{\prime \prime}(t)-u^{\prime}(t)\right), \quad \beta_{j}^{*}=\bar{\sigma}_{u}^{j}\left(t, x^{u}(t, \omega), u(t, \omega)\right)\left(u^{\prime \prime}(t)-u^{\prime}(t)\right)$. 


$$
\left\|q^{u^{\prime \prime}, u}(., .)-q^{u^{\prime}, u}(., .)\right\|_{2}^{*} \leq \breve{D}^{* *}\left(1+n^{\prime}\right) M^{+}\left|u^{\prime \prime}(\breve{s}, \omega)-u^{\prime}(\breve{s}, \omega)\right|_{2} .
$$

In (25) and (26), $\breve{D}^{* *}$ is independent of $u^{\prime \prime}, u^{\prime}, u$ (it only depends on $M^{+}$). We need to prove that

$$
\left\|\pi \dot{x}^{u^{\prime}}(t, .)-\pi \dot{x}^{u}(t, .)\right\|_{2} \rightarrow 0 \text { when }\left\|u^{\prime}-u\right\|_{2} \rightarrow 0 .
$$

This follows from (24) and (2), because, in a shorthand notation, for all $t$,

$$
\begin{aligned}
& \left|\pi f\left(t, x^{u^{\prime}}, u^{\prime}\right)-\pi f\left(t, x^{u}, u\right)\right|_{2} \\
& \leq\left|\pi f\left(t, x^{u^{\prime}}, u^{\prime}\right)-\pi f\left(t, x^{u}, u^{\prime}\right)\right|_{2}+\left|\pi f\left(t, x^{u}, u^{\prime}\right)-\pi f\left(t, x^{u}, u\right)\right|_{2} \\
& \leq M^{+}\left|x^{u^{\prime}}-x^{u}\right|_{2}+M^{+}\left|u^{\prime}-u\right|_{2} \\
& \leq M^{+}\left(\left\|x^{u^{\prime}}-x^{u}\right\|_{2}+\left\|u^{\prime}-u\right\|_{2}\right) \\
& \leq M^{+} D M^{+}\left(1+n^{\prime}\right)\left\|u^{\prime}-u\right\|_{2}+M^{+}\left\|u^{\prime}-u\right\|_{2} .
\end{aligned}
$$

We also need:

$$
\left\|\pi \dot{q}^{u^{\prime \prime}, u}(t, .)-\pi \dot{q}^{u^{\prime}, u}(t, .)\right\|_{2} \rightarrow 0 \text { when }\left\|u^{\prime \prime}-u^{\prime}\right\|_{2} \rightarrow 0 .
$$

This follows from (26) and (2), because, in a shorthand notation, for $f_{x}=f_{x}\left(t, x^{u}, u\right), f_{u}=f_{u}\left(t, x^{u}, u\right)$, for all $t$,

$$
\begin{aligned}
& \left|\pi f_{x} q^{u^{\prime \prime}, u}(t, .)+\pi f_{u}\left(u^{\prime \prime}-u\right)-\pi f_{x} q^{u^{\prime}, u}(t, .)-\pi f_{u}\left(u^{\prime}-u\right)\right|_{2} \\
& \leq\left|\pi f_{x}\left(q^{u^{\prime \prime}, u}(t, .)-q^{u^{\prime}, u}(t, .)\right)\right|_{2}+\left|\pi f_{u}\left(u^{\prime \prime}-u^{\prime}\right)\right|_{2} \\
& \leq M^{+}\left|\left(q^{u^{\prime \prime}, u}(t, .)-q^{u^{\prime}, u}(t, .)\right)\right|_{2}+M^{+}\left|u^{\prime \prime}-u^{\prime}\right|_{2} \\
& \leq M^{+} \breve{D}^{* *}\left(1+n^{\prime}\right) M^{+}\left\|u^{\prime \prime}-u^{\prime}\right\|_{2}+M^{+}\left\|u^{\prime \prime}-u^{\prime}\right\|_{2} .
\end{aligned}
$$

Note finally that, when $u_{*} \in U_{*}, \quad \hat{u}, u^{\prime} \in \mathrm{cl}_{2} U^{* *}$, then

$$
\begin{aligned}
& f_{u}\left(., x^{u_{*}}(., .), u_{*}(., .)\right) \hat{u}(., .), f\left(., x^{u^{\prime}}(., .), u^{\prime}(., .)\right)-f\left(., x^{u^{*}}(., .), u^{*}(.)\right) \\
& \text { and } f_{x}\left(., . x^{u_{*}}(., .), u_{*}(., .)\right) q^{u^{\prime}, u_{*}}(., .) \text { belong to } \mathrm{cl}_{2} L_{2}^{* n^{*}},
\end{aligned}
$$

see Remark L in Appendix.

B) Local controllability of the linear perturbations.

Note that, by (2), in a shorthand notation,

$$
\left\|\pi f_{u}\left(., x^{u_{*}}, u_{*}\right)\left[\hat{u}-u^{*}\right]-\pi f_{u}\left(., x^{u_{*}}, u_{*}\right)\left[\hat{u}-u_{*}\right]\right\|_{2} \leq M^{+}\left\|u_{*}-u^{*}\right\|_{2} \leq \alpha / 4
$$

when $\left\|u_{*}-u^{*}\right\|_{2} \leq \alpha / 4 M^{+}, \hat{u}, u_{*} \in U_{*}$. Then, from (15) and (29), when

$$
\begin{aligned}
u_{*} \in\left\{u \in c l_{2} U^{* *}:\left\|u-u^{*}\right\|_{2} \leq \delta_{*}^{*}:=\min \left\{1, \delta_{*}, \alpha / 4 M^{+}\right\}\right\}, \\
1_{[c, 1]}\left[\breve{z}(., .)+B_{3 \alpha / 4}^{* m^{*}}\right] \subset \operatorname{cl}_{2} \operatorname{co}\left\{1_{[c, 1]}\left[\pi f_{u}\left(., x^{u_{*}}, u_{*}\right)\left[\hat{u}-u_{*}\right]\right]: \hat{u} \in U^{* *}\right\} \subset L_{2}^{*, m^{*}},
\end{aligned}
$$

where "co" can be omitted due to the concavity of $U$. To see this, apply Remark $\mathrm{W}$ in Appendix.

Next, let the number $c^{\prime} \in[c, 1)$ satisfy $M^{+2}\left(1+n^{\prime}\right) 2 \breve{D}^{* *}\left(1-c^{\prime}\right)^{1 / 2} \leq \alpha / 6$, (for $\breve{D}^{* *}$, see (25)), and let 
$\mu_{*}:=\min \left\{1, \delta_{*}, \alpha\left(1-c^{\prime}\right) / 64 M^{+}, \alpha\left(1-c^{\prime}\right) / 64 M^{+2}\left(1+n^{\prime}\right) \breve{D}^{* *}\right\} \leq \delta_{*}^{*}$. Let

$u_{*} \in U_{*}^{*}:=\left\{u \in \mathrm{cl}_{2} U^{* *}:\left\|u-u^{*}\right\|_{2} \leq \mu_{*}\right\}$, so $\left\|u_{*}-u^{*}\right\|_{2} \leq 1$. When

$u \in U^{c^{\prime}}:=\left\{u \in U^{* *}: u=u^{*}\right.$ on $\left.\left[0, c^{\prime}\right)\right\}$, then $\left\|u-u^{*}\right\|_{2} \leq 1$, hence $\left\|u_{*}-u\right\|_{2} \leq 2$.

Then, for all $u \in U^{c^{\prime}}, u_{*} \in U_{*}^{*}$, for all $t$

$$
\left|\pi f_{x}\left(t, x^{u_{*}}, u_{*}\right) q^{u, u_{*}}\right|_{2} \leq \alpha\left(1-c^{\prime}\right) / 64+1_{\left[c^{\prime}, 1\right]}(t) \alpha / 8 \leq \alpha / 3 .
$$

To see this, note that by (25), for all $t$,

$$
\begin{aligned}
& \left.\pi f_{x}\left(t, x^{u_{*}}(t, .), u_{*}(t, .)\right) q^{u, u_{*}}(t, .)\right|_{2} \\
\leq & M^{+}\left|q^{u, u_{*}}(t, .)\right|_{2} \\
\leq & M^{+} M^{+}\left(1+n^{\prime}\right) \breve{D}^{* *}\left(E \int_{0}^{t}\left|u(t, .)-u_{*}(t, .)\right|^{2} \mathrm{~d} t\right)^{1 / 2} \\
\leq & M^{+} M^{+}\left(1+n^{\prime}\right) \breve{D}^{* *}\left\{\left[E \int_{0}^{c^{\prime}}\left(\left|u^{*}(t, .)-u_{*}(t, .)\right|\right)^{2} \mathrm{~d} t\right]^{1 / 2}\right. \\
& \left.+1_{\left[c^{\prime}, 1\right]}(t)\left[E \int_{c^{\prime}}^{1}\left(\left\|u(. . .)-u_{*}(. . .)\right\|_{2}\right)^{2} \mathrm{~d} t\right]^{1 / 2}\right\} \\
\leq & M^{+^{2}}\left(1+n^{\prime}\right) \breve{D}^{* *}\left|u^{*}-u_{*}\right|_{2}+1_{\left[c^{\prime}, 1\right]}(t) M^{+^{2}}\left(1+n^{\prime}\right) 2 \breve{D}^{* *}\left(1-c^{\prime}\right)^{1 / 2} \\
\leq & \alpha\left(1-c^{\prime}\right) / 64+1_{\left[c^{\prime}, 1\right]}(t) \alpha / 6 \leq \alpha / 3 .
\end{aligned}
$$

Then, using again Remark $\mathrm{W}$ in Appendix and (31), (29), (32), for all $u_{*} \in U_{*}^{*}$,

$$
\begin{aligned}
& 1_{\left[c^{\prime}, 1\right]}\left[\breve{z}(., .)+B_{\alpha / 2}^{* * *}\right] \\
& \subset \operatorname{cl}_{2}\left\{1_{\left[c^{\prime}, 1\right]}\left[\pi f_{x}\left(., x^{u_{*}}, u_{*}\right) q^{\hat{u}, u_{*}}+\pi f_{u}\left(., x^{u_{*}}, u_{*}\right)\left[\hat{u}-u_{*}\right]\right]: \hat{u} \in U^{c^{\prime}}\right\} .
\end{aligned}
$$

C) Relations between exact and linear perturbations.

Let $u^{\prime \prime}, u$ be given elements in $U_{*}$. Let $\varepsilon^{\prime}>0$ be arbitrarily given. Let us first prove that for $r \in(0,1]$ small enough, for any $\delta \in(0, r]$, for $u^{\prime}=\delta u^{\prime \prime}+(1-\delta) u$, for all $t$,

$$
\left|\delta^{-1}\left(x^{u^{\prime}}(t, .)-x^{u}(t, .)-\delta q^{u^{\prime \prime}, u}(t, .)\right)\right|_{2} \leq \varepsilon^{\prime} .
$$

Write $\bar{\sigma}_{x} q \mathrm{~d} B_{t}=\sum_{j} \bar{\sigma}_{x}^{j} q \mathrm{~d} B_{t}^{j}, \quad \bar{\sigma}_{u} u \mathrm{~d} B_{t}=\sum_{j} \bar{\sigma}_{u}^{j} u \mathrm{~d} B_{t}^{j}$. Define, in a shorthand notation,

$$
\begin{aligned}
& \xi^{1}(t, \omega)= \delta^{-1}\left\{f\left(t, x^{u}+\delta q^{u^{\prime \prime}, u}, u^{\prime}\right)-f\left(t, x^{u}, u\right)-f_{x}\left(t, x^{u}, u\right) \delta q^{u^{\prime \prime}, u}\right. \\
&\left.-f_{u}\left(t, x^{u}, u\right)\left[\delta\left(u^{\prime \prime}-u\right)\right]\right\}, \\
& \xi_{1}(t)=\left[E\left(\int_{0}^{t} \xi^{1}(s) \mathrm{d} s\right)^{2}\right]^{1 / 2}, \\
& \xi^{2}(t, \omega):= \delta^{-1}\left\{\bar{\sigma}\left(t, x^{u}+\delta q^{u^{\prime \prime}, u}, u^{\prime}\right)-\bar{\sigma}\left(t, x^{u}, u\right)-\bar{\sigma}_{x}\left(t, x^{u}, u\right) \delta q^{u^{\prime \prime}, u}\right. \\
&-\left.\bar{\sigma}_{u}\left(t, x^{u}, u\right)\left[\delta\left(u^{\prime \prime}-u\right)\right]\right\},
\end{aligned}
$$




$$
\xi_{2}(t):=\left\{E\left(\int_{0}^{t} \xi^{2}(s) \mathrm{d} B_{s}\right)^{2}\right\}^{1 / 2}
$$

For any $\varepsilon^{\prime \prime}>0$, there exists a $\delta^{\prime} \in(0,1]$, such that

$$
\xi_{i}(t) \leq \varepsilon^{\prime \prime}, i=1,2 \text {, when } \delta \in\left(0, \delta^{\prime}\right] \text {, uniformly in } t \text {, }
$$

by Lemmas B and C in Appendix.

Consider now

$$
\begin{aligned}
& \delta^{-1}\left(x^{u^{\prime}}(t)-x^{u}(t)-\delta q^{u^{\prime \prime}, u}(t)\right) \\
& =\int_{0}^{t} \delta^{-1}\left\{f\left(s, x^{u^{\prime}}, u^{\prime}\right)-f\left(s, x^{u}, u\right)-f_{x}\left(s, x^{u}, u\right) \delta q^{u^{\prime \prime}, u}-\delta f_{u}\left(s, x^{u}, u\right)\left(u^{\prime \prime}-u\right)\right\} \mathrm{d} s \\
& +\int_{0}^{t} \delta^{-1}\left[\bar{\sigma}\left(s, x^{u^{\prime}}, u^{\prime}\right)-\bar{\sigma}\left(s, x^{u}, u\right)-\bar{\sigma}_{x}\left(s, x^{u}, u\right) \delta q^{u^{\prime \prime}, u}-\delta \bar{\sigma}_{u}\left(s, x^{u}, u\right)\left(u^{\prime \prime}-u\right)\right] \mathrm{d} B_{s} .
\end{aligned}
$$

By (56) in Appendix, Lemma A, for $\delta \in\left(0, \delta^{\prime}\right]$, for some $\bar{K}$ only dependent on $M^{+}$, for all $t$

$$
\left\|\delta^{-1}\left(x^{u^{\prime}}(t)-x^{u}(t)-\delta q^{u^{\prime \prime}, u}(t)\right)\right\|_{2} \leq \bar{K} \varepsilon^{\prime \prime},
$$

To see this, in Lemma A let $\breve{z}=x^{u^{\prime}}, \quad \breve{h}=f\left(t, x, u^{\prime}\right), \quad \breve{y}=x^{u}+\delta q^{u^{\prime \prime}, u}$, $\left(-\delta \xi^{1}\right)=\beta:=-f\left(t, \breve{y}, u^{\prime}\right)+f\left(t, x^{u}, u\right)+f_{x}\left(t, x^{u}, u\right) \delta q^{u^{\prime \prime}, u}+f_{u}\left(t, x^{u}, u\right) \delta\left[u^{\prime \prime}-u\right]$, $\alpha^{*}=\alpha=0, \sigma_{*}=\bar{\sigma}\left(t, x, u^{\prime}\right)$,

$\left(-\delta \xi^{2}\right)=\beta^{*}:=-\bar{\sigma}\left(t, \breve{y}, u^{\prime}\right)+\bar{\sigma}\left(t, x^{u}, u\right)+\bar{\sigma}_{x}\left(t, x^{u}, u\right) \delta q^{u^{\prime \prime}, u}+\bar{\sigma}_{u}\left(t, x^{u}, u\right) \delta\left[u^{\prime \prime}-u\right]$. To obtain (34), put $\varepsilon^{\prime \prime}=\varepsilon^{\prime} / \bar{K}$.

Next, given $u^{\prime \prime}, u \in U_{*}$ and assume that $\left\|u^{\prime \prime}-u\right\|_{2}^{*}<\infty$. Let us prove that for any $\varepsilon>0$, for $r \in(0,1]$ small enough, for any $\delta \in(0, r]$, for $u^{\prime}=\delta u^{\prime \prime}+(1-\delta) u \in U_{*}$, we have that

$$
\left\|\delta^{-1}\left[\pi \dot{x}^{u^{\prime}}-\pi \dot{x}^{u}-\delta \pi \dot{q}^{u^{\prime \prime}, u}\right]\right\|_{2} \leq \varepsilon .
$$

Let

$$
\begin{aligned}
\left(\pi \xi^{1}(t, \omega)=\right) \xi^{3}(t, \omega):= & \delta^{-1}\left\{\pi f\left(t, x+\delta q^{u^{\prime \prime}, u}, u^{\prime}\right)-\pi f\left(t, x^{u}, u\right)\right. \\
& \left.-\pi f_{x}\left(t, x^{u}, u\right) \delta q^{u^{\prime \prime}, u}-\pi f_{u}\left(t, x^{u}, u\right)\left[\delta\left(u^{\prime \prime}-u\right)\right]\right\}
\end{aligned}
$$

Now,

$$
\begin{aligned}
\left|\xi^{3}(t, \omega)\right| \leq & \delta^{-1} \sup _{\theta \in[0,1)} \mid\left\{\pi f_{x}\left(t, x^{u}+\delta \theta q^{u^{\prime \prime}, u}, \theta\left(u^{\prime}-u\right)\right) \delta q^{u^{\prime \prime}, u}\right. \\
& +\pi f_{u}\left(t, x^{u}+\delta \theta q^{u^{\prime \prime}, u}, \theta\left(u^{\prime}-u\right)\right) \delta\left(u^{\prime \prime}-u\right)-\pi f_{x}\left(t, x^{u}, u\right) \delta q^{u^{\prime \prime}, u} \\
& \left.-\pi f_{u}\left(t, x^{u}, u\right)\left[\delta\left(u^{\prime \prime}-u\right)\right]\right\} \mid=: \xi^{4}(t, \omega) .
\end{aligned}
$$

Because $\left\|q^{u^{\prime \prime}, u}\right\|_{2}^{*}<\infty$ and $\left\|u^{\prime \prime}-u\right\|_{2}^{*}<\infty$, then Lemma J in Appendix gives that $\left\|\xi^{4}(., .)\right\|_{2} \rightarrow 0 \quad$ when $\quad \delta \rightarrow 0$. Next, let $\xi_{*}=\delta^{-1}\left[\pi \dot{x}^{u^{\prime}}-\pi \dot{x}^{u}-\delta \pi \dot{q}^{u^{\prime \prime}, u}\right]$, $\xi_{* *}=\pi f\left(t, x^{u^{\prime}}, u^{\prime}\right)-\pi f\left(t, x^{u}+\delta q^{u^{\prime \prime}, u}, u^{\prime}\right)$. Using $\left|\xi_{* *}\right| \leq M^{+}\left|x^{u^{\prime}}-x^{u}-\delta q^{u^{\prime \prime}, u}\right|$ and $\xi_{*}=\xi_{* *}+\xi^{3}$, we get $\left\|\xi_{*}\right\|_{2} \leq M^{+} \delta^{-1}\left\|x^{u^{\prime}}-x^{u}-\delta q^{u^{\prime \prime}, u}\right\|_{2}+\left\|\xi^{4}\right\|_{2}$, which $\rightarrow 0$ when $\delta \rightarrow 0$, by (36). Hence, (37) follows. 
D) Continuity of $u \rightarrow q^{u^{\prime}, u}$ at $u^{*}$.

Let $u, u^{\prime} \in U_{*}$. Define $\delta(t, \omega):=q^{u^{\prime}, u}(t, \omega)-q^{u^{\prime}, u^{*}}(t, \omega)$. Let us first prove that

$$
\|\delta(., .)\|_{2} \rightarrow 0 \text { when }\left|u-u^{*}\right|_{2} \rightarrow 0 .
$$

Now, in a shorthand notation,

$$
\begin{aligned}
\delta(t)= & \int_{0}^{t}\left\{f_{x}\left(s, x^{u}, u\right) q^{u^{\prime}, u}+f_{u}\left(s, x^{u}, u\right)\left[u^{\prime}-u\right]\right\} \mathrm{d} s \\
& -\int_{0}^{t}\left\{f_{x}\left(s, x^{*}, u^{*}\right) q^{u^{\prime}, u^{*}}+f_{u}\left(s, x^{*}, u^{*}\right)\left[u^{\prime}-u^{*}\right]\right\} \mathrm{d} s \\
& +\int_{0}^{t}\left\{\bar{\sigma}_{x}\left(s, x^{u}, u\right) q^{u^{\prime}, u}+\int_{0}^{t}\left\{\bar{\sigma}_{u}\left(s, x^{u}, u\right)\left[u^{\prime}-u\right]\right\} \mathrm{d} B_{s}\right. \\
& -\int_{0}^{t}\left\{\bar{\sigma}_{x}\left(s, x^{u^{*}}, u^{*}\right) q^{u^{\prime}, u^{*}}+\bar{\sigma}_{u}\left(s, x^{u^{*}}, u^{*}\right)\left[u^{\prime}-u^{*}\right]\right\} \mathrm{d} B_{s} \\
= & \int_{0}^{t}\left[f_{x}\left(s, x^{u}, u\right) q^{u^{\prime}, u}-f_{x}\left(s, x^{u}, u\right) q^{u^{\prime}, u^{*}}\right] \mathrm{d} s \\
& +\int_{0}^{t}\left[f_{x}\left(s, x^{u}, u\right)-f_{x}\left(s, x^{*}, u^{*}\right)\right] q^{u^{\prime}, u^{*}} \mathrm{~d} s \\
& +\int_{0}^{t}\left[f_{u}\left(s, x^{u}, u\right)-f_{u}\left(s, x^{*}, u^{*}\right)\right]\left[u^{\prime}-u\right] \mathrm{d} s \\
& +\int_{0}^{t} f_{u}\left(s, x^{*}, u^{*}\right)\left[u^{*}-u\right] \mathrm{d} s \\
& +\sum_{j} \int_{0}^{t}\left[\bar{\sigma}_{x}^{j}\left(s, x^{u}, u\right) q^{u^{\prime}, u}-\bar{\sigma}_{x}^{j}\left(s, x^{u}, u\right) q^{u^{\prime}, u^{*}}\right] \mathrm{d} B_{s}^{j} \\
& +\sum_{j} \int_{0}^{t}\left[\bar{\sigma}_{x}^{j}\left(s, x^{u}, u\right)-\bar{\sigma}_{x}^{j}\left(s, x^{*}, u^{*}\right)\right] q^{u^{\prime}, u^{*}} \mathrm{~d} B_{s}^{j} \\
& +\sum_{j} \int_{0}^{t}\left[\bar{\sigma}_{u}^{j}\left(s, x^{u}, u\right)-\bar{\sigma}_{u}^{j}\left(s, x^{*}, u^{*}\right)\right]\left[u^{\prime}-u\right] d B_{s} \\
& +\sum_{j} \int_{0}^{t} \bar{\sigma}_{u}^{j}\left(s, x^{*}, u^{*}\right)\left[u^{*}-u\right] \mathrm{d} B_{s} .
\end{aligned}
$$

Using the notation in Lemma A in Appendix, we write

$$
\begin{aligned}
\delta(t)=\int_{0}^{t} \breve{h}(s, \delta) \mathrm{d} s+\int_{0}^{t} \sigma_{*}(s, \delta) \mathrm{d} B_{s}+\int_{0}^{t} \alpha \mathrm{d} s+\int_{0}^{t} \alpha_{*} \mathrm{~d} B_{s} \text { where } \\
\breve{h}(s, \delta)=f_{x}\left(s, x^{u}, u\right) \delta, \sigma_{*}^{j}(s, \delta)=\bar{\sigma}_{x}^{j}\left(s, x^{u}, u\right) \delta, \\
\alpha=\left[f_{x}\left(s, x^{u}, u\right)-f_{x}\left(s, x^{*}, u^{*}\right)\right] q^{u^{\prime}, u^{*}} \\
+\left[f_{u}\left(s, x^{u}, u\right)-f_{u}\left(s, x^{*}, u^{*}\right)\right]\left[u^{\prime}-u\right]+f_{u}\left(s, x^{*}, u^{*}\right)\left[u^{*}-u\right], \\
\alpha_{j}^{*}=\left[\bar{\sigma}_{x}^{j}\left(s, x^{u}, u\right)-\bar{\sigma}_{x}^{j}\left(s, x^{*}, u^{*}\right)\right] q^{u^{\prime}, u^{*}} \\
+\left[\bar{\sigma}_{u}^{j}\left(s, x^{u}, u\right)-\bar{\sigma}_{u}^{j}\left(s, x^{*}, u^{*}\right)\right]\left[u^{\prime}-u\right]+\bar{\sigma}_{u}^{j}\left(s, x^{*}, u^{*}\right)\left[u^{*}-u\right], \\
\left(\breve{z}=\delta, \breve{y} \equiv 0, \quad \beta=\beta^{*}=0\right) .
\end{aligned}
$$

Then, by (57),

$$
\left(E\left(\sup _{s \leq t} \delta(s)\right)^{2}\right)^{1 / 2} \leq \breve{D}^{*}\left\{\left(E \int_{0}^{t}|\alpha|^{2} \mathrm{~d} s\right)^{1 / 2}+\sum_{j}\left(E \int_{0}^{t}\left|\alpha_{j}^{*}\right|^{2} \mathrm{~d} s\right)^{1 / 2}\right\}
$$

where

$$
\begin{aligned}
\left(E \int_{0}^{t}|\alpha|^{2} \mathrm{~d} s\right)^{1 / 2} \leq & \left(E \int_{0}^{t}\left(e(s, u)\left|q^{u^{\prime}, u^{*}}\right|\right)^{2} \mathrm{~d} s\right)^{1 / 2}+\left(E \int_{0}^{t}\left(e^{*}(s, u)\left|u^{\prime}-u\right|\right)^{2} \mathrm{~d} s\right)^{1 / 2} \\
& +\left(E \int_{0}^{t} M^{+2}\left|u^{*}-u\right|^{2} \mathrm{~d} s\right)^{1 / 2}
\end{aligned}
$$


for

$$
e(s, u):=\left|f_{x}\left(s, x^{u}, u\right)-f_{x}\left(s, x^{*}, u^{*}\right)\right|, e^{*}=\left|f_{u}\left(s, x^{u}, u\right)-f_{u}\left(s, x^{*}, u^{*}\right)\right|,
$$

and

$$
\begin{aligned}
\left(E \int_{0}^{t}\left|\alpha_{j}^{*}\right|^{2} \mathrm{~d} s\right)^{1 / 2} \leq & \left(E \int_{0}^{t}\left(e_{j}(s, u)\left|q^{u^{\prime}, u^{*}}\right|\right)^{2} \mathrm{~d} s\right)^{1 / 2}+\left(E \int_{0}^{t}\left(e_{j}^{*}(s, u)\left|u^{\prime}-u\right|\right)^{2} \mathrm{~d} s\right)^{1 / 2} \\
& +\left(E \int_{0}^{t} M^{+2}\left|u^{*}-u\right|^{2} \mathrm{~d} s\right)^{1 / 2}
\end{aligned}
$$

for

$$
e_{j}(s, u)=\left|\sigma_{x}^{j}\left(s, x^{u}, u\right)-\sigma_{x}^{j}\left(s, x^{*}, u^{*}\right)\right|, e_{j}^{*}=\left|\bar{\sigma}_{u}^{j}\left(s, x^{u}, u\right)-\bar{\sigma}_{u}^{j}\left(s, x^{*}, u^{*}\right)\right| .
$$

Consider e.g the term

$\left(E \int_{0}^{t}\left(e_{j}(s, u)\left|q^{u^{\prime}, u^{*}}\right|\right)^{2} \mathrm{~d} s\right)^{1 / 2} \leq\left(E \int_{0}^{1}\left(e_{j}(s, u)\left|q^{u^{\prime}, u}\right|\right)^{2} \mathrm{~d} s\right)^{1 / 2}$. Now,

$e_{j}\left|q^{u^{\prime}, u^{*}}\right| \leq 2 M^{+}\left|q^{u^{\prime}, u^{*}}\right|, \quad q^{u^{\prime}, u^{*}} \in L_{2}\left(J \times \Omega, \mathbb{R}^{n}\right)$, and $\left|x^{u}-x^{*}\right|_{2} \rightarrow 0$ when $\left|u-u^{*}\right|_{2} \rightarrow 0$, Hence, by Remark T in Appendix, $\left(E \int_{0}^{1}\left(e_{j}(s, u)\left|q^{u^{\prime}, u^{*}}\right|\right)^{2} \mathrm{~d} s\right)^{1 / 2} \rightarrow 0$ when $\left|u-u^{*}\right|_{2} \rightarrow 0$. Similarly, the terms containing $e, e^{*}, e_{j}^{*}$ converge to zero when $\left|u-u^{*}\right|_{2} \rightarrow 0$. So $\left(E\left(\sup _{s \leq t} \delta(s)\right)^{2}\right)^{1 / 2} \rightarrow 0$ uniformly in $t$ when $\left|u-u^{*}\right|_{2} \rightarrow 0$.

Next, assume that $\left\|u^{\prime}-u^{*}\right\|_{2}^{*}<\infty, u^{\prime}, u \in U_{*}$. We want to prove that then $\delta_{*}$ satisfies

$$
\left\|\delta_{*}(., .)\right\|_{2} \rightarrow 0 \text { when }\left\|u-u^{*}\right\|_{2} \rightarrow 0 .
$$

where, in a shorthand notation,

$$
\begin{aligned}
\delta_{*}(t, \omega): & \pi f_{x}\left(t, x^{u}, u\right) q^{u^{\prime}, u}+\pi f_{u}\left(t, x^{u}, u\right)\left[u^{\prime}-u\right] \\
& -\pi f_{x}\left(t, x^{*}, u^{*}\right) q^{u^{\prime}, u^{*}}+\pi f_{u}\left(t, x^{*}, u^{*}\right)\left[u^{\prime}-u^{*}\right]
\end{aligned}
$$

Now,

$$
\begin{aligned}
\delta_{*}(t, \omega)= & {\left[\pi f_{x}\left(t, x^{u}, u\right) q^{u^{\prime}, u}-\pi f_{x}\left(t, x^{u}, u\right) q^{u^{\prime}, u^{*}}\right] } \\
& +\left[\left\{\pi f_{x}\left(t, x^{u}, u\right)-\pi f_{x}\left(t, x^{*}, u^{*}\right)\right\} q^{u^{\prime}, u^{*}}\right] \\
& +\left[\pi f_{u}\left(t, x^{u}, u\right)\left(u^{\prime}-u\right)-\pi f_{u}\left(t, x^{u}, u\right)\left(u^{\prime}-u^{*}\right)\right] \\
& +\left[\pi f_{u}\left(t, x^{u}, u\right)\left(u^{\prime}-u^{*}\right)-\pi f_{u}\left(t, x^{*}, u^{*}\right)\left(u^{\prime}-u^{*}\right)\right]
\end{aligned}
$$

Let us show that the four terms in square brackets have $\|\cdot\|_{2}$-norms $\rightarrow 0$ when $\left\|u-u^{*}\right\|_{2} \rightarrow 0$. Now, $\left\|x^{u}-x^{*}\right\|_{2} \rightarrow 0$ when $\left\|u-u^{*}\right\|_{2} \rightarrow 0$. Hence, by Lemma $J$ in Appendix, the second and fourth square bracket $\rightarrow 0$ in $\|\cdot\|_{2}$ when $\left\|u-u^{*}\right\|_{2} \rightarrow 0$ (recall that $\left\|u^{\prime}-u^{*}\right\|_{2}^{*}<\infty$ and $\left.\left\|q^{u^{\prime}, u}\right\|_{2}^{*}<\infty\right)$. Moreover the first and third square bracket have $|$.$| -norms smaller than$ $2 M^{+}\left|q^{u^{\prime}, u}-q^{u^{\prime} u^{*}}\right|$ and $2 M^{+}\left|u^{*}-u\right|$, respectively. So the $\|\cdot\|_{2}$-norms of both 
these terms $\rightarrow 0$ when $\left\|u-u^{*}\right\|_{2} \rightarrow 0$, recall that $\left\|q^{u^{\prime}, u}-q^{u^{\prime}, u^{*}}\right\|_{2} \rightarrow 0$ when $\left\|u-u^{*}\right\|_{2} \rightarrow 0$, see (38). So (39) has been proved.

E) Dense subsets of $\mathrm{Cl}_{2} U^{* *}$.

Let $u \in \mathrm{Cl}_{2} L_{\infty}^{\text {prog }}\left(J \times \Omega, \mathbb{R}^{n^{* *}}\right)=L_{2}^{*, n^{* * *}}$ (for this equality see Appendix L). Note that for each $\varepsilon>0$, for some $u^{\prime} \in L_{\infty}^{\text {prog }}\left(J \times \Omega, \mathbb{R}^{n^{\prime \prime}}\right),\left\|u^{\prime}-u\right\|_{2}<\varepsilon$. Let $M=\operatorname{essup}_{t, \omega}\left|u^{\prime}(t, \omega)\right|$. For each $(t, \omega)$, let $u^{\prime \prime}(t, \omega)$ be the projection of $u^{\prime}(t, \omega)$ on the line containing 0 and $u(t, \omega)$. Let $u_{*}(t, \omega)=0$ if $u^{\prime \prime}(t, \omega)$ has the opposite direction of $u(t, \omega)$ or if $u(t, \omega)=0$, let $u_{*}(t, \omega)=u(t, \omega)$ if $u^{\prime \prime}(t, \omega)$ is longer than $u(t, \omega)$ and has the same direction as $u(t, \omega)$, and if $u^{\prime \prime}(t, \omega)$ is smaller than $u(t, \omega)$ and has the same direction as $u(t, \omega)$, let $u_{*}(t, \omega)=u^{\prime \prime}(t, \omega)$. Evidently, for some measurable $\phi_{u}(t, \omega) \in[0,1]$, $u_{*}(t, \omega)=\phi_{u}(t, \omega) u(t, \omega)$, and $\left|\phi_{u}(t, \omega) u(t, \omega)-u(t, \omega)\right| \leq\left|u^{\prime}(t, \omega)-u(t, \omega)\right|$ and $\left|\phi_{u}(t, \omega) u(t, \omega)\right| \leq\left|u^{\prime \prime}(t, \omega)\right| \leq\left|u^{\prime}(t, \omega)\right| \quad\left(\phi_{u}\right.$ also depends on $u^{\prime}$, the notation hides this fact). Evidently, $\operatorname{essup}_{t, \omega}\left|\phi_{u}(t, \omega) u(t, \omega)\right| \leq M$ and $\phi_{u}(t, \omega)$ is progressively measurable. Now, for any $u_{* *} \in \mathrm{cl}_{22} U^{* *}$, let $U_{u_{* *}}=\left\{u_{* *}+w: w \in\left(\mathrm{cl}_{2} U^{* *}-u_{* *}\right) \cap L_{\infty}^{\text {prog }}\left(J \times \Omega, \mathbb{R}^{n^{* *}}\right)\right\} \subset \mathrm{cl}_{2} U^{* *}$. We have that $U_{u_{* *+}}$ is $\|\cdot\|_{2}$-dense in $\mathrm{cl}_{2} U^{* *}$ because if $\hat{u} \in \mathrm{cl}_{2} U^{* * *}$, for any $\varepsilon>0$, for some $u^{\prime} \in L_{\infty}^{\text {prog }}\left(J \times \Omega, \mathbb{R}^{n^{* * *}}\right),\left\|\left(\hat{u}-u_{* *}\right)-u^{\prime}\right\|_{2}<\varepsilon \quad\left(\hat{u}, u_{* *}\right.$, and hence, $\hat{u}-u_{* *}$, belong to the linear space $\left.\mathrm{Cl}_{2} L_{\infty}^{\text {prog }}\left(J \times \Omega, \mathbb{R}^{n^{* *}}\right)\right)$. Then

$\left\|\phi_{\hat{u}-u_{* *}}(t, \omega)\left(\hat{u}(t, \omega)-u_{* *}(t, \omega)\right)-\left(\hat{u}(t, \omega)-u_{* *}(t, \omega)\right)\right\|_{2}<\varepsilon$, and so

$\left\|u_{* *}+w-\hat{u}\right\|_{2}<\varepsilon$ for

$w=\phi_{\hat{u}-u_{* *}}(t, \omega)\left(\hat{u}(t, \omega)-u_{* *}(t, \omega)\right) \in\left(\mathrm{cl}_{2} U^{* *}-u_{* *}\right) \cap L_{\infty}\left(J \times \Omega, \mathbb{R}^{n^{* *}}\right) \quad\left(\mathrm{cl}_{2} U^{* *} \quad\right.$ is convex).

Note finally that if $u^{\prime \prime}, u \in U^{* *}$, then $u^{\prime \prime}=u^{*}+w^{\prime \prime}, u=u^{*}+w$ for $w^{\prime \prime}, w \in U^{*}$, so $\left\|u^{\prime \prime}-u\right\|_{2}=\left\|w-w^{\prime \prime}\right\|_{2} \leq\|w\|_{2}+\left\|w^{\prime \prime}\right\|_{2} \leq 2$, in fact

$$
u^{\prime \prime}, u \in \mathrm{cl}_{2} U^{* *} \Rightarrow\left\|u^{\prime \prime}-u\right\|_{2} \leq 2 \text {. }
$$

F) Final proof steps.

Define

$$
I_{i}:=\left(1-1 / 2^{i}, 1-1 / 2^{i+1}\right], i=0,1, \cdots,
$$

Using Jensens inequality, for an arbitrary function $\phi \geq 0$ we get $\left\{[1 / \text { meas }(I)] \int_{I} \phi \mathrm{d} t\right\}^{2} \leq\left\{[1 / \text { meas }(I)] \int_{I} \phi^{2} \mathrm{~d} t\right\}^{2}$. It follows that

$$
\begin{aligned}
2^{i}\left(E\left(\int_{I_{i}} \phi \mathrm{d} t\right)^{2}\right)^{1 / 2} & =2^{i}\left[E\left(2^{-(i+1)} \int_{I_{i}} \phi^{2} \mathrm{~d} t\right)\right]^{1 / 2}=2^{i}\left[2^{-(i+1)} \int_{I_{i}} E \phi^{2} \mathrm{~d} t\right]^{1 / 2} \\
& \leq 2^{i}\left[2^{-(i+1)} \int_{I_{i}}\left(\|\phi\|_{2}\right)^{2} \mathrm{~d} t\right]^{1 / 2} \leq(1 / 2)\|\phi\|_{2} .
\end{aligned}
$$

For $z(.) \in L_{2}\left(\Omega, \Phi, \mathbb{R}^{m^{*}}\right)$, define $\Pi_{1}(z()):.=E\left[z() \mid. \Phi_{1-1 / 2}\right]$ and $\Pi_{i}(z()):.=E\left[z() \mid. \Phi_{1-1 / 2^{i}}\right]-E\left[z() \mid. \Phi_{1-1 / 2^{i-1}}\right], i>1$. Define 


$$
\begin{gathered}
{ }_{2}|z(.)|:=\sup _{i} 2^{i}\left|\Pi_{i} z(.)\right|_{2} \text { for } z(.) \in L_{2}\left(\Omega, \Phi, \mathbb{R}^{m^{*}}\right), \\
B^{\infty}=\left\{z(., .) \in L_{2}^{\text {prog }}\left(\Omega, \Phi, \mathbb{R}^{m^{*}}\right):\|z(., .)\|_{2}<\infty\right\} .
\end{gathered}
$$

Furthermore, let $L^{2}$ be the subset of $L_{2}\left(\Omega, \Phi, \mathbb{R}^{m^{*}}\right)$ consisting of all element $z(.) \in L_{2}\left(\Omega, \Phi, \mathbb{R}^{m^{*}}\right)$ such that ${ }_{2}|z()|:.=\sup _{i} 2^{i}\left|\Pi_{i} z(.)\right|_{2}<\infty$ and such that $z()=.\lim _{k \rightarrow \infty} \sum_{1 \leq i \leq k} \Pi_{i} z()=.\lim _{k \rightarrow \infty} E\left[z() \mid. \Phi_{1-1 / 2^{k}}\right]$, (limit in $|\cdot|_{2}$-norm, $\left.\sum_{1 \leq i \leq k} \Pi_{i} z()=.E\left[z() \mid. \Phi_{1-1 / 2^{k}}\right]\right)$. It is easily seen that elements of the type $\int_{J} y(t, \omega) \mathrm{d} t, \quad y(t, \omega) \in L_{2}^{\text {prog }}\left(J \times \Omega, \mathbb{R}^{m^{*}}\right),\|y(., .)\|_{2}<\infty$, precisely make up the set $L^{2}$. To see this, let $y(.,$.$) be such a function, and, using Jensen's$ inequality three times (and for any $\alpha(t) \geq 0$, that $\left.\operatorname{essup}_{t}(\alpha(t))^{1 / 2}=\left(\operatorname{essup}_{t} \alpha(t)\right)^{1 / 2}\right)$, note that for any interval $J^{\prime}$,

$$
\begin{aligned}
& \left(E\left[E\left[\int_{J^{\prime}}|y(t, .)| \mathrm{d} t \mid \Phi_{1-1 / 2^{k}}\right]\right]^{2}\right)^{1 / 2} \leq\left(E\left[E\left[\left(\int_{J^{\prime}}|y(t, .)| \mathrm{d} t\right)^{2} \mid \Phi_{1-1 / 2^{k}}\right]\right]\right)^{1 / 2} \\
& \leq\left(E\left[\operatorname{meas}\left(J^{\prime}\right) \int_{J^{\prime}}|y(t, .)|^{2} \mathrm{~d} t\right]\right)^{1 / 2}=\left(\operatorname{meas}\left(J^{\prime}\right)\right)^{1 / 2}\left(E\left[\int_{J^{\prime}}|y(t, .)|^{2} \mathrm{~d} t\right]\right)^{1 / 2} \\
& \leq\left(\operatorname{meas}\left(J^{\prime}\right)\right)^{1 / 2}\left(\int_{J^{\prime}} \operatorname{essup}_{t} E|y(t, .)|^{2} \mathrm{~d} t\right)^{1 / 2} \\
& =\operatorname{meas}\left(J^{\prime}\right)\left(\operatorname{essup}_{t} E|y(t, .)|^{2}\right)^{1 / 2}=\operatorname{meas}\left(J^{\prime}\right)\|y(. . .)\|_{2},
\end{aligned}
$$

so, in particular, $\left|\Pi_{1} \int_{J} y(t, .) \mathrm{d} t\right|_{2} \leq\|y(., .)\|_{2}$. This yields also, for $j>1$, that

$$
\begin{aligned}
& \left|\Pi_{j} \int_{J} y(t, .) \mathrm{d} t\right|_{2} \\
& =\left|\Pi_{j} \sum_{0 \leq i<\infty} \int_{I_{i}} y(t, .) \mathrm{d} t\right|_{2}=\left|\sum_{j-1 \leq i<\infty} \Pi_{j} \int_{I_{i}} y(t, .) \mathrm{d} t\right|_{2} \\
& \leq\left|\sum_{j-1 \leq i<\infty} E\left[\int_{I_{i}} y(t, .) \mathrm{d} t \mid \Phi_{1-1 / 2^{j}}\right]\right|_{2}+\left|\sum_{j-1 \leq i<\infty} E\left[\int_{I_{i}} y(t, .) \mathrm{d} t \mid \Phi_{1-1 / 2^{j-1}}\right]\right|_{2} \\
& \leq 2 \cdot \sum_{j-1 \leq i<\infty}\left(1 / 2^{i+1}\right)\|y(., .)\|_{2}=2 \cdot\left(1 / 2^{j-1}\right)\|y(., .)\|_{2},
\end{aligned}
$$

so $\quad 2\left|\int_{J} y(t, \omega) \mathrm{d} t\right| \leq 4\|y(., .)\|_{2}<\infty$, see (43). Moreover, by (44), similarly,

$$
\begin{aligned}
& \left|\int_{J} y(t, \omega) \mathrm{d} t-\sum_{1 \leq j \leq k} \Pi_{j} \int_{J} y(t, \omega) \mathrm{d} t\right|_{2} \\
& =\left|\int_{J} y(t, \omega) \mathrm{d} t-E\left[\int_{J} y(t, \omega) \mathrm{d} t \mid \Phi_{1-1 / 2^{k}}\right]\right|_{2} \\
& =\left|\int_{J} y(t, \omega) \mathrm{d} t-\int_{0}^{1-1 / 2^{k}} y(t, \omega) \mathrm{d} t-E\left[\int_{1-1 / 2^{k}}^{1} y(t, \omega) \mathrm{d} t \mid \Phi_{1-1 / 2^{k}}\right]\right|_{2} \\
& \leq\left(2 / 2^{k}\right)|| y(. . .) \|_{2},
\end{aligned}
$$

so $\int_{J} y(t, \omega) \mathrm{d} t$ is a $L_{2}$-limit of $\sum_{1 \leq j \leq k} \Pi_{j} \int_{J} y(t, \omega) \mathrm{d} t$. Hence,

$\int_{J} y(t, \omega) \mathrm{d} t \in L^{2}$. Note also that $\operatorname{essup}_{t, \omega}|y(t, \omega)|<\infty \Rightarrow$ 


$$
\begin{aligned}
& \operatorname{essup}_{\omega} 2^{i}\left|\Pi_{i} \int_{J} y(t, \omega) \mathrm{d} t\right| \\
& =\operatorname{essup}_{\omega} 2^{i}\left|\Pi_{i} \int_{1-1 / 2^{i-1}}^{1} y(t, \omega) \mathrm{d} t\right| \\
& \leq \operatorname{essup}_{\omega} 2^{i}\left|E\left[\int_{1-1 / 2^{i-1}}^{1} y(t, \omega) \mathrm{d} t \mid \Phi_{1-1 / 2^{i}}\right]\right| \\
& +2^{i}\left|E\left[\int_{1-1 / 2^{i-1}}^{1} y(t, \omega) \mathrm{d} t \mid \Phi_{1-1 / 2^{i-1}}\right]\right| \\
& \leq 4 \operatorname{essup}_{t, \omega}|y(t, \omega)|<\infty
\end{aligned}
$$

for all $i>1$ (holding "the more" for $i=1$ ).

Finally, if $z(\omega) \in L^{2}$, then $z(\omega)=\int_{J} \gamma(t, \omega) \mathrm{d} t=\lim _{k \rightarrow \infty} \int_{0}^{1-1 / 2^{k+1}} \gamma(t, \omega) \mathrm{d} t$, for

$$
\gamma(t, .):=2 \sum_{m \geq 1} 2^{m} \Pi_{m} z(.) 1_{\left[1-1 / 2^{m}, 1-1 / 2^{m+1}\right)}(t)=: \Psi z(.),
$$

where $\sup _{m} 2^{m}\left|\Pi_{m} z(\omega)\right|_{\infty}=(1 / 2) \operatorname{essup}_{t, \omega}|\gamma(t, \omega)|$,

and where $\|\gamma(. . .)\|_{2} \leq 2 \cdot{ }_{2}|z()|,. \gamma(.,$.$) progressively measurable. (So the linear$ map $\Psi$ satisfies $\left.\|\Psi z(.)\|_{2} \leq 2 \cdot{ }_{2}|z()|.\right)$

Let $\Theta$ be the linear map from $B^{\infty}$ (see (43)) into $L_{2}\left(\Omega, \Phi, \mathbb{R}^{m^{*}}\right)$ defined by $z(.,.) \rightarrow \int_{0}^{1} z(t, \omega) \mathrm{d} t$ and note that $\Theta \Psi z()=.z(),. z(.) \in L^{2}$. In (45) we have just proved that $\Theta$ has norm $\leq 4$ for the norms $\|\cdot\|_{2}$ and ${ }_{2}|\cdot|$ (or for $\|\cdot\|_{2} \rightarrow{ }_{2}|\cdot|$, as we shall express it).

Now, let $L_{\infty}^{*}=\left\{z(.) \in L^{2}: \sup _{m}\left|2^{m} \Pi_{m} z(.)\right|_{\infty}<\infty\right\}$, let $\mathrm{cl}_{(2)}$ be closure in $L^{2}$ in ${ }_{2}|\cdot|$-norm, let $B_{(2)}^{*}(0, \beta)=\left\{z(.) \in L_{\infty}^{*}:{ }_{2}|z()|<.\beta\right\}$, let

$B_{(2)}^{\infty}(0, \beta):=\mathrm{cl}_{(2)} B_{(2)}^{*}(0, \beta)$, and let $B_{2}^{\infty}(0, \beta)$ be a $\|\cdot\|_{2}$-ball in $L_{\infty}^{\text {prog }}\left(J \times \Omega, \mathbb{R}^{m^{*}}\right)$.

Now, for $z \in B_{(2)}^{*}\left(0, \alpha\left(1-c^{\prime}\right) / 4\right)$, we have $z=\int_{c^{\prime}}^{1} \gamma^{\prime}(s,.) \mathrm{d} s$, where

$$
\gamma^{\prime}(s, \omega)=2\left(1-c^{\prime}\right)^{-1} \sum_{i=1}^{\infty} 2^{i} \Pi_{i} z 1_{\left[1-\left(1-c^{\prime}\right) / 2^{i}, 1-\left(1-c^{\prime}\right) / 2^{i+1}\right)} \in B_{2}^{\infty}(0, \alpha / 2) .
$$

Hence, by (33), using $L_{2}^{* m^{*}}=\mathrm{cl}_{2} L_{\infty}^{\text {prog }}\left(J \times \Omega, \mathbb{R}^{m^{*}}\right)$ and $B_{2}^{\infty}(0, \alpha / 2) \subset B_{\alpha / 2}^{* m^{*}}$, for $u_{*} \in U_{*}^{*}$, (for $U_{*}^{*}$ and $U^{c^{\prime}}$, used in a moment, see definitions subsequent to (31)), we get

$$
\begin{aligned}
& \int_{c^{\prime}}^{1} \breve{d} t+B_{(2)}^{*}\left(0, \alpha\left(1-c^{\prime}\right) / 4\right) \\
& \subset \int_{c^{\prime}}^{1} \breve{Z} \mathrm{~d} t+\int_{c^{\prime}}^{1} B_{2}^{\infty}(0, \alpha / 2) \mathrm{d} t \subset \int_{c^{\prime}}^{1} \mathrm{cl}_{2} Q \mathrm{~d} t \subset \mathrm{cl}_{(2)} \int_{c^{\prime}}^{1}, \mathrm{~d} t,
\end{aligned}
$$

where

$$
Q:=\left\{\pi f_{x}\left(t, x^{u_{*}}, u_{*}\right) q^{\hat{u}, u_{*}}+\pi f_{u}\left(t, x^{u_{*}}, u^{*}\right)\left[\hat{u}-u_{*}\right]: \hat{u} \in U^{c^{\prime}}\right\}
$$

(note that $\gamma^{\prime \prime}(.,.) \rightarrow \int_{c^{\prime}}^{1}, \gamma^{\prime \prime}(s,.) \mathrm{d} s$ is continuous in $\|\cdot\|_{2} \rightarrow{ }_{2}|$.$| , as shown above).$ Let $u \in U^{c^{\prime}}, u_{*} \in U_{*}^{*}$. Then

$$
\begin{aligned}
\pi q^{u, u_{*}}(1)= & \int_{0}^{c^{\prime}}\left\{\pi f_{x}\left(t, x^{u_{*}}, u_{*}\right) q^{u^{*}, u_{*}}+\pi f_{u}\left(t, x^{u_{*}}, u_{*}\right)\left(u^{*}-u_{*}\right)\right\} \mathrm{d} t \\
& +\int_{c^{\prime}}^{1}\left\{\pi f_{x}\left(t, x^{u_{*}}, u_{*}\right) q^{u, u_{*}}+\pi f_{u}\left(t, x^{u_{*}}, u_{*}\right)\left(u-u_{*}\right)\right\} \mathrm{d} t .
\end{aligned}
$$

Using Remark W, $|\Theta| \leq 4$, and the fact that the first integrand has a $\|$.$\| -$ norm $\leq \alpha\left(1-c^{\prime}\right) / 64+M^{+}\left\|u^{*}-u_{*}\right\|_{2} \leq \alpha\left(1-c^{\prime}\right) / 64+\alpha\left(1-c^{\prime}\right) / 64=\alpha\left(1-c^{\prime}\right) / 32$ 
by (32) (hence the first integral has a ${ }_{2}|\cdot|$-norm $\leq \alpha\left(1-c^{\prime}\right) / 8$, we get from (49) that

$$
\int_{c^{\prime}}^{1} \breve{z} \mathrm{~d} t+B_{(2)}^{\infty}\left(0, \alpha\left(1-c^{\prime}\right) / 8\right) \subset \mathrm{cl}_{(2)} q^{U^{c^{\prime}}, u_{*}}(1) .
$$

Observe that, given $u \in \mathrm{cl}_{2} U^{* *}, u^{\prime \prime} \in U_{u} \quad\left(\Rightarrow\left|u^{\prime \prime}-u\right|_{\infty}<\infty \Rightarrow\left\|u^{\prime \prime}-u\right\|_{2}^{*}<\infty\right)$, by $|\Theta| \leq 4$ for $\|\cdot\|_{2} \rightarrow{ }_{2}|\cdot|$ and (37), for any given $\varepsilon>0$, for $r \in(0,1]$ small enough, for any $\delta \in(0, r]$,

$$
\delta^{-1} \cdot\left|\pi x^{\delta u^{\prime \prime}+(1-\delta) u}(1, .)-\pi x^{u}(1, .)-\delta \pi q^{u^{\prime \prime} u}(1, .)\right| \leq \varepsilon
$$

And, by (39) and $|\Theta| \leq 4$, for $u^{\prime} \in U_{u^{*}}\left(\Rightarrow\left|u^{\prime}-u^{*}\right|_{\infty}<\infty \Rightarrow\left\|u^{\prime}-u^{*}\right\|_{2}^{*}<\infty\right)$, then

$$
{ }_{2}\left|\pi q^{u^{\prime}, u}(1, .)-\pi q^{u^{\prime}, u^{*}}(1, .)\right| \rightarrow 0 \text { when }\left\|u-u^{*}\right\|_{2} \rightarrow 0, u \in \mathrm{cl}_{2} U^{* *} .
$$

To obtain the conclusion in Theorem 1, we will now apply Corollary I in Appendix, and for this end, we make the following identifications: $\hat{Y}=\mathrm{cl}_{(2)} L_{\infty}^{*}$, $\gamma^{*}=\mu^{*}$ (for $\mu^{*}$ see below), $\tilde{K}_{*}^{\prime}=2, \tilde{A}=\mathrm{cl}_{2} U^{* *}, \bar{\sigma}^{*}=\|\cdot\|_{2}$, the norm $|$.$| on$ $\hat{Y} \quad$ equal to ${ }_{2}|\cdot|, \quad \tilde{a}^{+}=u^{\prime \prime}, \quad \tilde{a}^{\prime}=u^{\prime}, \quad \tilde{a}^{*}=u^{*}, \quad \tilde{a}=u, \quad \tilde{A}_{\tilde{a}}=U_{u}$, $\hat{H}(\tilde{a})=\Theta \pi \dot{x}^{u}-\Theta \pi \dot{x}^{u^{*}}, \quad \hat{q}^{\tilde{a}^{\prime}, \tilde{a}}=\Theta \pi \dot{q}^{u^{\prime}, u}, \quad \breve{H}(\tilde{a})=E\left[a \cdot x^{u}(1,).\right]$, $\widetilde{q}^{\tilde{a}^{\prime}, \tilde{a}}=E\left[a \cdot q^{u^{\prime}, u}(1,).\right], \quad \hat{y}=0$. By (34) and (51) it follows that the property (61) is satisfied for $\tilde{a}^{\prime}=\delta \tilde{a}^{+}+(1-\delta) \tilde{a}$ for $\tilde{a}^{+} \in \tilde{A}_{\tilde{a}}\left(\left\|u^{\prime}-u\right\|_{2} \leq 2 \delta\right.$ by (40)), and (62) is trivially satisfied by concavity of $U$, both (61) and (62) in the manner required in Corollary I. By (50), for $\breve{Z}^{*}:=\int_{C^{\prime}}^{1} \breve{z}(t,.) \mathrm{d} t$, $B_{(2)}^{\infty}\left(\breve{Z}^{*}, \alpha\left(1-c^{\prime}\right) / 8\right) \subset \mathrm{cl}_{(2)} \operatorname{co} \pi q^{\mathrm{cl}_{2} U^{* *}, u}(1,$.$) for u \in U_{*}^{*}$, so for $\varepsilon=\alpha\left(1-c^{\prime}\right) / 8$, $\left.B\left(\breve{z}^{*}, \varepsilon\right) \subset \operatorname{clco} \hat{q}^{\tilde{A}, \tilde{a}} \subset \hat{Y}, \quad \tilde{a} \in \operatorname{cl} B\left(\tilde{a}^{*}, \mu^{*}\right)\right)$, where $\mu^{*}:=\mu_{*}, \quad \mu_{*}$ defined subsequent to (31). By $|\Theta| \leq 4$ and (27) and (24), $\hat{H}$ and $\breve{H}$ are continuous. By (26), (28), and $|\Theta| \leq 4, \quad \tilde{a}^{\prime} \rightarrow \tilde{q}^{\tilde{a}^{\prime}, \tilde{a}}$ is continuous in $\tilde{A}$, for any $\tilde{a} \in \tilde{A}$, and by (38) and (52), for $\tilde{a}^{\prime}=u^{\prime} \in \tilde{A}_{\tilde{a}^{*}}=U_{u^{*}}=U^{* *}, \quad \tilde{a} \rightarrow \tilde{q}^{\tilde{a}^{\prime}, \tilde{a}}$ is continuous at $\tilde{a}^{*}=u^{*}$. The required boundedness of $\breve{q}^{a^{\prime}, a}$ is satisfied because of (25). Evidently, $\tilde{A}$ is complete, see Appendix, Remark M. Hence all conditions in Corollary I are satisfied. Thus, for some $\Lambda_{0} \geq 0$, some ${ }_{2}|\cdot|$-continuous linear functional $v$ on $\mathrm{cl}_{(2)} L_{\infty}^{*},\left(\Lambda_{0}, v\right) \neq 0$, for all $u \in \mathrm{cl}_{2} U^{* *}$, $\Lambda_{0} E\left[a \cdot q^{u, u^{*}}(1,).\right]+\left\langle\pi q^{u, u^{*}}(1,), v.\right\rangle \leq 0$.

By Hahn-Banach's theorem, $v$ has an ${ }_{2}|\cdot|$-continuous linear extension to all $L^{2}$, also denoted $v$.

Proof of Remark 1. Let $T=1$. Note that (50) and $\breve{Z}^{*}=0$ gives $B_{(2)}^{\infty}\left(0, \alpha\left(1-c^{\prime}\right) / 8\right) \subset \mathrm{cl}_{(2)} \operatorname{co}\left\{\pi q^{\mathrm{cl}_{2} U^{* *}, u^{*}}(1)\right\}$. If $\Lambda_{0}=0$, the necessary condition (16) implies $\left\langle\mathrm{Cl}_{(2)} \operatorname{co}\left\{\pi q^{\mathrm{cl}_{2} U^{* *}, u^{*}}(1)\right\}, v\right\rangle \leq 0$ and hence $\left\langle B_{(2)}^{\infty}\left(0, \alpha\left(1-c^{\prime}\right) / 8\right), v\right\rangle \leq\left. 0 \Rightarrow v\right|_{\mathrm{cl}_{(2)} L_{\infty}^{*}}=0$, a contradiction. So $\Lambda_{0} \neq 0$.

From (16), in a shorthand notation, we get, for $w \in U^{*}$, and even for $w \in \tilde{U}=\left\{\lambda u: \lambda \geq 0, u \in \mathrm{cl}_{2} U^{*}\right\}$, that

$$
\left\langle\int_{0}^{1} \pi f_{u} w \mathrm{~d} t, v\right\rangle \leq-\Lambda_{0}\left\langle\int_{0}^{1} E\left(f_{x} q^{w}+f_{u} w\right) \mathrm{d} t, a\right\rangle-\left\langle\int_{0}^{1} \pi f_{x} q^{w} \mathrm{~d} t, v\right\rangle .
$$


Fix any $\beta(.,.) \in L_{\infty}^{\text {prog }}\left(J \times \Omega, \mathbb{R}^{m^{*}}\right)$, such that

$|\beta|_{2}=1+\left\|f_{u}\left(t, x^{*}(t, \omega), u^{*}\right) u^{*}(t, \omega)\right\|_{2}+\left\|u^{*}(t, \omega)\right\|_{2}=: \theta$. By (21) and $\left\|u^{*}\right\|_{2}^{*}<\infty$, there exists a $w^{\prime} \in U^{\prime} \cap \mathrm{cl}_{2} L_{\infty}^{\text {prog }}\left(J \times \Omega, \mathbb{R}^{n^{* *}}\right)$, such that

$$
\begin{aligned}
& \beta(t, \omega)+\pi f_{u}\left(t, x^{*}(t, \omega), u^{*}(t, \omega)\right) u^{*}(t, \omega)=\pi f_{u}\left(t, x^{*}(t, \omega), u^{*}(t, \omega)\right) w^{\prime}(t, \omega), \\
& \left|w^{\prime}(t, \omega)\right| \leq \beta_{0}\left|\beta(t, \omega)+f_{u}\left(t, x^{*}(t, \omega), u^{*}(t, \omega)\right) u^{*}(t, \omega)\right| . \text { So } \\
& \beta=\pi f_{u}\left(t, x^{*}(t, \omega), u^{*}(t, \omega)\right) w \text {, where } w=w^{\prime}-u^{*}, \\
& |w(t, \omega)| \leq \beta_{0}\left|\beta(t, \omega)+f_{u}\left(t, x^{*}(t, \omega), u^{*}(t, \omega)\right) u^{*}(t, \omega)\right|+\left|u^{*}(t, \omega)\right| \text {, so } w \in \tilde{U} \\
& \left(\|w\|_{2} \leq \beta_{0}|\beta|_{\infty}+\beta_{0} \theta\right) \text { and }|w|_{2} \leq \beta_{0}|\beta(t, \omega)|_{2}+\beta_{0}\left|f_{u} u^{*}\right|_{2}+\left|u^{*}\right|_{2} \leq\left(2 \beta_{0}+1\right) \theta .
\end{aligned}
$$

By (25), for some constant $K$, $\left|\operatorname{essup}_{t} q^{w}\right|_{2} \leq K|w|_{2} \leq K\left(2 \beta_{0}+1\right) \theta$, so the absolute value of the scalar products on the right hand side of (53) are smaller than $|a| M^{+} K|w|_{2}+M^{+}|w|_{2} \quad\left(\leq \theta_{*} \theta, \theta_{*}:=|a|\left(M^{+} K+M^{+}\right)\left(2 \beta_{0}+1\right)\right) \quad$ and $|v| \cdot \int_{2}\left|\int_{0}^{1} \pi f_{x} q^{w} \mathrm{~d} t\right|$, respectively, the last number being small than $|v| 4\left\|\pi f_{x} q^{w}\right\|_{2} \leq 4|v| M^{+} K|w|_{2} \leq 4|v| M^{+}\left(2 \beta_{0}+1\right) \theta$. Hence, by (53), for $K^{\prime}:=\theta_{*}+4|v| M^{+}\left(2 \beta_{0}+1\right),\left\langle\int_{0}^{1} \beta \mathrm{d} t, v\right\rangle \leq K^{\prime} \theta$. Replacing $\beta$ by $-\beta$, by the same argument, if $|-\beta|_{2}=\theta$, we get $\left\langle\int_{0}^{1}-\beta \mathrm{d} t, v\right\rangle \leq K^{\prime} \theta$. In fact, we have $\left|\left\langle\int_{0}^{1} \beta \mathrm{d} t, v\right\rangle\right| \leq K^{\prime} \theta$ for all $\beta \in L_{\infty}^{\text {prog }}\left(J \times \Omega, \mathbb{R}^{m^{*}}\right),|\beta|_{2}=\theta$.

Thus, on this subspace of $L_{2}\left(J \times \Omega, \Phi_{1}, \mathbb{R}^{m^{*}}\right), \quad \beta \rightarrow\left\langle\int_{0}^{1} \beta \mathrm{d} t, v\right\rangle$ is $|\cdot|_{2}$ continuous. Hence, by Hahn-Banach's theorem (see [8]) and a representation theorem, for some $v^{* *}(t, \omega)$ in $L_{2}\left(J \times \Omega, \Phi_{1}, \mathbb{R}^{m^{*}}\right)$, $\left\langle\int_{0}^{1} \beta \mathrm{d} t, v\right\rangle=E \int_{0}^{1}\left\langle\beta, v^{* *}\right\rangle \mathrm{d} t$, for all $\beta \in L_{\infty}^{\text {prog }}\left(J \times \Omega, \mathbb{R}^{m^{*}}\right) \quad$ (in fact on $L_{2}^{\text {prog }}\left(J \times \Omega, \mathbb{R}^{m^{*}}\right)$.

Note that for $\beta \in \bigcup_{t} L_{\infty}^{\text {prog }}\left(\Omega, \Phi_{t}, R^{m^{*}}\right)$,

$\langle\beta, v\rangle=\left\langle\int_{0}^{1} \beta \mathrm{d} s, v\right\rangle=E\left\langle\int_{0}^{1} \beta \mathrm{d} s, v^{* *}(s,).\right\rangle \mathrm{d} s=E\left\langle\beta, \int_{0}^{1} v^{* *}(s,).\right\rangle \mathrm{d} s$, which means that $v$ is $|\cdot|_{2}$-continuous on $\bigcup_{t} L_{\infty}^{\text {prog }}\left(\Omega, \Phi_{t}, R^{m^{*}}\right)$ and has a unique $|\cdot|_{2}$-continuous extension to the $|\cdot|_{2}$-closure of $\bigcup_{t} L_{\infty}^{\text {prog }}\left(\Omega, \Phi_{t}, R^{m^{*}}\right)$, which equals $L_{2}\left(\Omega, \Phi_{1}, R^{m^{*}}\right)$ (and includes $L^{2}$ ) in case of left continuity of $t \rightarrow \Phi_{t}$.

\section{Proof of Remark 2.}

Let $T=1, \quad x_{0}=0$. Let $v_{*}:=\phi^{\prime} \rightarrow\left\langle\phi^{\prime}, v\right\rangle+\Lambda_{0} E\left\langle\phi^{\prime}, a\right\rangle \quad\left(\phi^{\prime} \in L^{2}\right)$ and let $C(1, t, \omega)^{*}$ and $\pi^{*}$ be the adjoints of $z \rightarrow C(1, t, \omega) z$ and of $\pi$.

It is easily proved (using Lemma A in Appendix and $|\Theta| \leq 4$ for $\|\cdot\|_{2} \rightarrow{ }_{2}|\cdot|$ ) that for $y(.) \in L_{2}\left(\Omega, \Phi_{s}, \mathbb{R}^{n^{*}}\right), s<1, \quad{ }_{2}|\pi C(1, s,) y.().| \leq D|y(.)|_{2}$ for some constant $D$, see Remark $\mathrm{P}$ in Appendix. Hence, for any given $s<1$, by the $|\cdot|_{2}-$ continuity of $y(.) \rightarrow\langle\pi C(1, s,) y.(), v$.$\rangle on L_{2}\left(\Omega, \Phi_{s}, \mathbb{R}^{n^{*}}\right), s<1$ and hence of $y(.) \rightarrow\left\langle C(1, s,) y.(),. v_{*}\right\rangle$, there exists a $L_{2}\left(\Omega, \Phi_{s}, \mathbb{R}^{n^{*}}\right)$-function $p^{-}(s, \omega)$ on $\Omega$ such that for any $L_{2}\left(\Omega, \Phi_{s}, \mathbb{R}^{n^{*}}\right)$-function $\alpha(\omega)$, we have $\left\langle C(1, s,.) \alpha(),. v_{*}\right\rangle=E\left\langle\alpha(),. p^{-}(s,).\right\rangle$. The function $p^{-}(s, \omega)$ is progressively 
measurable and right continuous in $s<1$, both a.s. and in $L_{2}$, see arguments in Remark Q in Appendix.

Assume now $\|u\|_{2}^{*}<\infty,(21)$ and left continuity of $t \rightarrow \Phi_{t}$, which implies $|\cdot|_{2}$-continuity of $v$ on all $L_{2}\left(\Omega, \Phi_{1}, \mathbb{R}^{m^{*}}\right)$ (by extension). In this case the argument for $|\cdot|_{2}$-continuity of $t \rightarrow p^{-}(t,$.$) for t<1$ in Remark $\mathrm{Q}$ in Appendix extends to $t=1$. Let $v_{* *}(.) \in L_{2}\left(\Omega, \Phi_{1}, \mathbb{R}^{m^{*}}\right)$ be a representation of $v$ and let $y(.,.) \in L_{\infty}^{p r o g}\left(J \times \Omega, R^{n^{*}}\right)$. Consider now

$$
\begin{aligned}
\left\langle\int_{0}^{1} \pi C(1, s, .) y(s, .) \mathrm{d} s, v\right\rangle & =E \int_{0}^{1}\left\langle\pi C(1, s, .) y(s, .), v_{* *}(.)\right\rangle \mathrm{d} s \\
& =E \int_{0}^{1}\left\langle y(s, .), C(1, s, .)^{*} \pi^{*} v_{* *}(.)\right\rangle \mathrm{d} s \\
& =\int_{0}^{1} E E\left[\left\langle y(s, .), C\left(1, s^{*}, .\right) \pi^{*} v_{* *}(.)\right\rangle \mid \Phi_{s}\right] \mathrm{d} s \\
& =E \int_{0}^{1}\left\langle y(s, .), E\left[C(1, s, .)^{*} \pi^{*} v_{* *}(.) \mid \Phi_{s}\right]\right\rangle \mathrm{d} s \\
& =E \int_{0}^{1}\left\langle y(s, .), p_{-}(s, .)\right\rangle \mathrm{d} s,
\end{aligned}
$$

where $p_{-}(s,)=.E\left[C(1, s, .)^{*} \pi^{*} v_{* *}() \mid. \Phi_{s}\right]$. In this case, we can evidently put $p^{-}(s,)=.E\left[C(1, s,.) \pi^{*} v_{* *}() \mid. \Phi_{s}\right]+\Lambda_{0}\left\langle E\left[C(1, s,) \mid. \Phi_{s}\right], a\right\rangle$ and we have

$$
\left\langle\int_{0}^{t} C(1, s, .) y(s, .) \mathrm{d} s, v_{*}\right\rangle=E \int_{0}^{t}\left\langle y(s, .), p^{-}(s, .)\right\rangle \mathrm{d} s, t<1 .
$$

In case of $\left\|u^{*}\right\|_{2}^{*}<\infty,(21)$, and left continuity of $\Phi_{t}$, by $|\cdot|_{2}$-continuity of $v_{*}$, the two definitions of $p^{-}(s)$ coincide, because for both definitions we have $\left\langle\int_{0}^{1} C(1, s,) y.(s,.) \mathrm{d} s, v_{*}\right\rangle=E \int_{0}^{1}\left\langle y(s,),. p^{-}(s,).\right\rangle \mathrm{d} s$ for all $y(.) \in 1_{[0, t]} L_{\infty}^{\text {prog }}\left(J \times \Omega, \mathbb{R}^{n^{*}}\right), t$ arbitrary in $[0,1)$.

Let now $\Phi_{t}$ be the natural filtration generated by the $B_{t}^{j}$ 's, augmented by the $P$-null sets in $\Phi:=\Phi_{1}$, and let $b \in[0,1)$.

Then, consider the pair of equations

$$
\begin{gathered}
\mathrm{d} p(t, \omega)=-p(t, \omega) f_{x}\left(t, x^{*}(t, \omega), u^{*}(t, \omega)\right) \mathrm{d} t \\
-\sum_{j} q^{j}(t) \sigma_{x}^{j}\left(t, x^{*}(t, \omega), u^{*}(t, \omega)\right) \mathrm{d} t+\sum_{j} q^{j}(t) \mathrm{d} B_{t}^{j}, \\
p(b, \omega)=p^{-}(b, \omega) .
\end{gathered}
$$

By Theorem 2.2, p. 349 in [6], there is a unique progressively measurable collection $p(t, \omega), q^{j}(t, \omega) \quad(t \in[0, b]), t \rightarrow p(t, \omega)$ continuous in $t \in[0, b]$, satisfying these equations (or more precisely, a.s. the integrated version of these equations), $\quad\left|p(., .) 1_{[0, b]}\right|_{2}<\infty, \quad\left|q^{j}(., .) 1_{[0, b]}\right|_{2}<\infty$, with $p(t, \omega)$ equal to $p^{-}(t, \omega)$-a.s. for each $t$ (see Remark $\mathrm{O}$ in Appendix). The uniqueness says that if two pairs $(p, q),\left(q=q^{1}, \cdots, q^{n^{\prime}}\right)$ and $(\hat{p}, \hat{q})$ satisfy the pair of equations, then $\operatorname{Pr}[p(t, \omega)=\hat{p}(t, \omega)$ for all $t \in[0, b]=1$ and $q(t, \omega)=\hat{q}(t, \omega)$ for a.e. $t \in[0, b]]=1$. We can let $b=b_{k} \uparrow 1$ when $k \rightarrow \infty \quad(k=1,2, \cdots)$ and obtain such functions $p_{k}(t, \omega), q_{k}(t, \omega)$ defined on each $\left[0, b_{k}\right]$. For $k^{\prime}<k$, by the fact that $p_{k^{\prime}}\left(b_{k^{\prime}}, \omega\right)=p^{-}\left(b_{k^{\prime}}, \omega\right)=p_{b_{k}}\left(b_{k^{\prime}}, \omega\right)$ a.s. and uniqueness, we have that $\left(p_{k^{\prime}}(t, \omega), q_{k^{\prime}}(t, \omega)\right)=\left(p_{k}(t, \omega), q_{k}(t, \omega)\right) \quad$ for $\quad$ a.e $t \in\left[0, b_{k^{\prime}}\right]$ a.s. Then, evidently, there exists a unique pair $(p(t, \omega), q(t, \omega))$ on $[0,1)$ satisfying (54), 
with $p(t, \omega)$ a.s. equal to $p^{-}(t, \omega)$ for any $t \in[0,1),\left|p(., .) 1_{[0, b]}\right|_{2}<\infty$, $\left|q^{j}(., .) 1_{[0, b]}\right|_{2}<\infty$ for all $b<1, t \rightarrow p(t, \omega)$ continuous.

Let us show (19). For any $s<1, q^{w}(1)=C(1, s) q^{w}(s)$ if $w=0$ on $(s, 1]$, so in this case

$0 \geq\left\langle q^{w}(1), v_{*}\right\rangle=\left\langle C(1, s,.) q^{w}(s), v_{*}\right\rangle=E\left\langle q^{w}(s), p^{-}(s)\right\rangle=E\left\langle q^{w}(s), p(s)\right\rangle$ consequence of (16)).

Define $f_{x}^{*}:=f_{x}^{*}(t,):.=f_{x}\left(t, x^{*}(t,),. u^{*}(t,).\right)$ and $f_{u}^{*}, \bar{\sigma}_{x}^{*}$, and $\bar{\sigma}_{u}^{*}$ similarly. It turns out that

$E\left\langle p(s,),. q^{w}(s,).\right\rangle=E \int_{0}^{s}\left\{p(t,.) f_{u}^{*}(t,) w.(t,)+.q(t,.) \bar{\sigma}_{u}^{*}(t,) w.(t,).\right\} \mathrm{d} t$. From this and $0 \geq E\left\langle p(s, .),. q^{w}(s, .).\right\rangle$ it follows that for all $u \in U$,

$p(t,.) f_{u}^{*}(t,).\left(u-u^{*}(t,).\right)+q(t,.) \bar{\sigma}_{u}^{*}(t,).\left(u-u^{*}(t,).\right) \leq 0$ for a.e. $t \in[0, s]$, a.s., see Remark S. In fact, this holds for a.e. $t \in[0,1]$ because $s<1$ was arbitrary. An informal proof, using Ito's formula, shows the last equality:

$$
\begin{aligned}
& E d\left\langle p, q^{w}\right\rangle=E\left\langle p, \mathrm{~d} q^{w}\right\rangle+E\left\langle\mathrm{~d} p, q^{w}\right\rangle+E\left\langle\mathrm{~d} p, \mathrm{~d} q^{w}\right\rangle \\
& =E\left\langle p, f_{x}^{*} q^{w} \mathrm{~d} t+f_{u}^{*} w \mathrm{~d} t\right\rangle+E\left\langle p,\left(\bar{\sigma}_{x}^{*} q^{w}+\bar{\sigma}_{u}^{*} w\right) \mathrm{d} B_{t}\right\rangle \\
& +E\left\langle-p f_{x}^{*} \mathrm{~d} t-q \bar{\sigma}_{x}^{*} \mathrm{~d} t+q \mathrm{~d} B_{t}, q^{w}\right\rangle+E\left[q \bar{\sigma}_{x}^{*} q^{w} \mathrm{~d} t\right]+E\left[q \bar{\sigma}_{u}^{*} w \mathrm{~d} t\right] \\
& =E\left[p f_{u}^{*} w \mathrm{~d} t+q \bar{\sigma}_{u}^{*} w \mathrm{~d} t\right] .
\end{aligned}
$$

So

$$
\begin{aligned}
& E\left\langle p(s, . .), q^{w}(s, . .)\right\rangle-E\left\langle p(0, .), q^{w}(0, .)\right\rangle=E\left\langle p(s, . .), q^{w}(s, . .)\right\rangle \\
& =E \int_{0}^{s}\left\{p(t, .) f_{u}^{*}(t, .) w(t, .)+q(t, .) \bar{\sigma}_{u}^{*}(t, .) w(t, .)\right\} \mathrm{d} t .
\end{aligned}
$$

Remark 4. (Exact attainability). In Theorem 1, drop the assumption that $u^{*}$ is optimal (and the optimization problem), so $\left(x^{*}, u^{*}\right)$ is simply a pair satisfying (4) and $\pi x^{*}(T,)=.\tilde{y}$ a.s. Then, for each $z(.,.) \in \operatorname{int}\left(\operatorname{cl}_{2} \operatorname{co}\left\{\pi \dot{q}^{u, u^{*}}: u \in \mathrm{cl}_{2} U^{* *}\right\}\right)$, $z(.,.) \in \mathrm{cl}_{2} L_{\infty}^{\text {prog }}\left(J \times \Omega, \mathbb{R}^{m^{*}}\right),\left(\mathrm{cl}_{2}\right.$ and int $=$ interior both corresponding to $\|\cdot\|_{2}$ and the space $\left.\mathrm{cl}_{(2)} L_{\infty}^{*}\right)$, for all $r>0$, for some number $\gamma \in(0, r]$ and some control $u \in \mathrm{Cl}_{2} U^{* *}, \pi x^{u}(T, \omega)=\tilde{y}+\gamma \int_{0}^{T} z(t, \omega) \mathrm{d} t$ a.s.

Proof of Remark 4. Let $T=1, x_{0}=0$. Corollary $\mathrm{G}$ in Appendix will be applied. Let $Y=\mathrm{cl}_{(2)} L_{\infty}^{*}, \quad \tilde{\mu}=\mu_{*}, \sigma=\|.\|_{2}$, the norm $|$.$| on Y$ equal to ${ }_{2}|$.$| ,$ $u^{\prime}=a^{\prime} \quad, \quad u^{*}=a^{*} \quad, \quad u=a \quad, \quad a^{+}=u^{\prime \prime} \quad, \quad A=\mathrm{cl}_{2} U^{* *}, \quad A_{a}=U_{u}$, $H(a)=\pi x^{u}(1,)-.\pi x^{*}(1,),. \quad q^{a^{\prime}, a}=\pi q^{u^{\prime}, u}(1,),. \quad \tilde{K}^{\prime}=2, \quad z^{*}=\breve{z}^{*}=\int_{c^{\prime}}^{1} \breve{z}(t,.) \mathrm{d} t$.

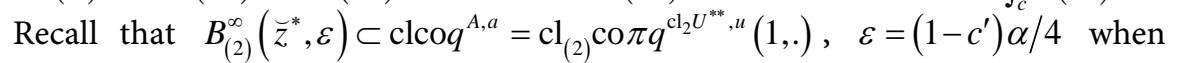
$u \in U_{*}^{*}$. By (51) it follows that the property (61) is satisfied for $a^{\prime}=\delta a^{+}+(1-\delta) a$ for $a^{+} \in A_{a}$, and (62) is trivially satisfied. By (27) $H$ is continuous, by (28), $a^{\prime} \rightarrow q^{a^{\prime}, a}$ is continuous in $A$ for any $a \in A$, and by (52), for $a^{\prime} \in A_{a}, a \rightarrow q^{a^{\prime}, a}$ is continuous at $a^{*}=u^{*}$.

Assume that $B_{\bar{\varepsilon}}^{* m^{*}}+\bar{z}(.,.) \subset \mathrm{cl}_{2} \operatorname{co} \pi \dot{q}^{\mathrm{cl}_{2} U^{* *}, u^{*}}(1,$.$) and let \bar{b}=\int_{J} \bar{z}(t,.) \mathrm{d} t$. For any $\hat{b} \in B_{(2)}^{*}(0, \bar{\varepsilon} / 2)$, it was shown earlier that there exists a $z^{\prime} \in L_{\infty}^{\text {prog }}\left(J \times \Omega, \mathbb{R}^{m^{*}}\right)$ such that $\hat{b}=\int_{J} z^{\prime}(t,.) \mathrm{d} t,\left\|z^{\prime}(., .)\right\|_{2} \leq 2 \cdot{ }_{2}|\bar{b}|<\bar{\varepsilon}$ 
$\left(z^{\prime}(.,)=.\Psi \hat{b}, \quad|\Psi| \leq 2, \quad z^{\prime}(.,.) \in B_{\bar{\varepsilon}}^{*, m^{*}}\right)$, so $B_{(2)}^{*}(\bar{b}, \bar{\varepsilon} / 2) \subset \mathrm{cl}_{2} \operatorname{co} \pi q^{\mathrm{cl}_{2} U^{* *}, u^{*}}(1,$.

and $B_{(2)}^{\infty}(\bar{b}, \bar{\varepsilon} / 2) \subset \mathrm{cl}_{2} \operatorname{co} \pi q^{\mathrm{cl}_{2} U^{* *}, u^{*}}(1,$.$) . Thus, if$

$z(.,.) \in \operatorname{int}\left(\operatorname{cl}_{2} \operatorname{co}\left\{\pi \dot{q}^{u, u^{*}}: u \in \mathrm{cl}_{2} U^{* *}\right\}\right)$, then

$b=\int_{J} z(t,.) \mathrm{d} t \in \operatorname{int} \mathrm{cl}_{(2)} \mathrm{co} \pi q^{\mathrm{cl}_{2} U^{* *}, u^{*}}(1,$.$) . Hence all conditions in Corollary \mathrm{G}$ are satisfied and the conclusion in Remark 4 follows.

Remark 5 If it is required that $\pi x(T,.) \in C$ instead of $\pi x(T,)=.\tilde{y}$, where $C$ is a ${ }_{2}\|\cdot\|$-closed convex set in $\mathrm{cl}_{(2)} L_{\infty}^{*}$, and if (15) holds for $\Gamma$ replaced by $\Gamma-\Psi C^{\prime}, \quad C^{\prime}:=\left\{z \in C-\pi x^{*}(T):{ }_{2}\left\|z-\pi x^{*}(T)\right\| \leq 1\right\}$ (for $\Psi$ see $(47)$ ), then (16) again holds, and with $\left\langle C-\pi x^{*}(1,), v.\right\rangle \geq 0$. In particular, this holds if $C=\left\{z \in \mathrm{cl}_{(2)} L_{\infty}^{*}: z^{j} \geq y^{j}\right.$ a.s, $j=1, \cdots, m^{* *} \leq m^{*}, \quad z^{j}=y^{j}$ a.s.,

$\left.j=m^{* *}+1, \cdots, m^{*}\right\}, \quad y^{j}$ given numbers. In fact, because $\pi x^{u}(T,.) \in \mathrm{Cl}_{(2)} L_{\infty}^{*}$ automatically, the results here cover the case where the terminal constraints are $x^{j}(T,.) \geq y^{j}$ a.s, $j=1, \cdots, m^{* *}, x^{j}(T,)=.y^{j}$ a.s., $j=m^{* *}+1, \cdots, m^{*}$.

Proof of Remark 5. A proof can be obtained (for $T=1$ ) by replacing $\tilde{A}=\mathrm{cl}_{(2)} U^{* *}$ by $\tilde{A} \times C^{\prime}$ and $\tilde{A}_{\tilde{a}}$ by $\tilde{A}_{\tilde{a}} \times C^{\prime}$, with $\hat{H}(\tilde{a})$ replaced by $\hat{H}(\tilde{a}, c)=\Theta \pi \dot{x}^{u}-\Theta \pi \dot{x}^{*}-c$, and $\hat{q}^{\tilde{q}^{\prime}, \tilde{a}}$ replaced by $\hat{q}^{\tilde{a}^{\prime}, \tilde{a}, c}=\Theta \pi \dot{q}^{u^{\prime}, u}-c$, keeping $\breve{H}$ and $\widetilde{q}^{\tilde{a}^{\prime}, \tilde{a}}$ as before and letting $\hat{y}=0, c^{*}=0$. (Then $\hat{H}(\tilde{a}, c)=0, \tilde{a}=u$, $c()=.c^{\prime}()-.\pi x^{*}(1,),. \quad c^{\prime} \in C$, gives

$\pi x^{u}(1,)-.\pi x^{*}(1,)-.\left(c^{\prime}()-.\pi x^{*}(1,).\right)=\pi x^{u}(1,)-.c^{\prime}()=$.0 , i.e.

$\left.c^{\prime}()=.\pi x^{u}(1,.) \in C\right)$. The details are omitted.

Remark 6 (The case $\pi \bar{\sigma}^{j} \neq 0$ for some or all $j, m^{*} \leq n^{*}$ ). Let $\mathrm{cl}_{\left.\right|_{||_{2}}}$ be closure in $L_{2}^{\text {prog }}\left(J \times \Omega, \mathbb{R}^{m^{*}}\right)$ as well as in $L_{2}\left(\Omega, \Phi_{T}, \mathbb{R}^{m^{*}}\right)$. For $u_{*} \in U_{*}$, let $U_{c^{\prime}, \bar{k}}^{u_{*}}:=\left\{w \in u_{*} 1_{\left[0, c^{\prime}\right)}+1_{\left[c^{\prime}, 1\right]} U^{\prime},\left|w-u_{*}\right|_{2} \leq \breve{k}\right\}$. When $\pi \bar{\sigma} \neq 0$, and $\Phi_{t}$ is the natural filtration, augmented by null sets as before, the necessary condition of Theorem 1 can be obtained for $v$ defined and continuous on $L_{2}\left(\Omega, \Phi_{T}, \mathbb{R}^{m^{*}}\right)$ (with $\Lambda_{0} \geq 0,\left(\Lambda_{0}, v\right) \neq 0$ ), if, for some $\breve{k}>0$, for some $c^{\prime}<1$, for some $z_{*}, z_{* *} \in L_{2}^{\text {prog }}\left(J \times \Omega, \mathbb{R}^{m^{*}}\right)$,

a) for some $\delta \in(0,1], \quad \beta_{*}>0,1_{\left[c^{\prime}, 1\right]}\left(B_{2}\left(z_{*}, \beta_{*}\right) \times B_{2}\left(z_{* *}, \beta_{*}\right)\right) \subset \mathrm{cl}_{|\cdot|_{2}} D_{u_{*}}$ when $\left|u_{*}-u^{*}\right|_{2} \leq \delta, \quad u_{*} \in U_{*}$, where

$$
\begin{aligned}
D_{u_{*}}:= & \left\{\left(1 _ { [ c ^ { \prime } , 1 ] } \left[\pi f_{x}\left(t, x^{u_{*}}(t, \omega), u_{*}(t, \omega)\right) q^{u, u_{*}}(t, \omega)\right.\right.\right. \\
& \left.+\pi f_{u}\left(t, x^{u_{*}}(t, \omega), u_{*}(t, \omega)\right)\left(u(t, \omega)-u_{*}(t, \omega)\right)\right], \\
& 1_{\left[c^{\prime}, 1\right]}\left[\pi \bar{\sigma}_{x}\left(t, x^{u_{*}}(t, \omega), u_{*}(t, \omega)\right) q^{u, u_{*}}(t, w)\right. \\
& \left.\left.\left.+\pi \bar{\sigma}_{u}\left(t, x^{u_{*}}(t, \omega), u_{*}(t, \omega)\right)\left(u(t, \omega)-u_{*}(t, \omega)\right)\right]\right): u \in U_{c^{\prime}, \bar{k}}^{u_{*}}\right\} .
\end{aligned}
$$

If $z_{*}=z_{* *}=0$, then $\Lambda_{0}>0$. The condition (A), with $z_{*}=z_{* *}=0$, is implied by

b): For some $\beta^{0}>0$, for all $(t, x, u)$, for any $y \in \mathbb{R}^{2 m^{*}}$,

$$
y \in\left\{\left(\pi f_{u}(t, x, u) w, \pi \bar{\sigma}_{u}(t, x, u) w\right): w \in U,|w| \leq \beta^{0}|y|\right\} .
$$


Letting $\xi:=1+\breve{k}+\left|u^{*}\right|_{2}, \quad \hat{Y}=L_{2}\left(\Omega, \Phi_{T}, \mathbb{R}^{m^{*}}\right)$, and $\tilde{A}=\tilde{A}_{a}=\tilde{U}:=\left\{u \in U^{\prime},|u|_{2} \leq \xi\right\}$, it is here sufficient to operate with the $|\cdot|_{2}$ -norm instead of ${ }_{2}|\cdot|$ on $\hat{Y}$, with $\bar{\sigma}^{*}=|\cdot|_{2}$. Note that $U_{c^{\prime}, \hat{k}}^{u_{*}} \subset \tilde{U}$, so $(A)$ holds for $U_{c^{\prime}, \tilde{k}}^{u_{*}}$ replaced by $\tilde{U}$. The set arising by replacing $U_{c^{\prime}, \tilde{k}}^{u_{*}}$ by $\tilde{U}$ in $D_{u_{*}}$ is denoted $\tilde{D}$. Define $z^{*}=\int_{C^{\prime}}^{1} Z_{*} \mathrm{~d} t+\int_{\mathcal{C}^{\prime}}^{1} Z_{* *} \mathrm{~d} B_{t}$. The condition (A), so modified, implies that for some $\delta^{\prime}>0, B_{2}^{\Omega}\left(z^{*}, \delta^{\prime}\right) \subset \mathrm{cl}_{|\cdot|_{2}} \operatorname{co}\left\{\int_{0}^{T} a \mathrm{~d} t+\int_{0}^{T} b \mathrm{~d} B_{t}:(a, b) \in \tilde{D}\right\}$, $u_{*}$ close to $u^{*}, B_{2}^{\Omega}\left(z^{*}, \delta^{\prime}\right)$ a ball in $L_{2}\left(\Omega, \Phi_{T}, \mathbb{R}^{m^{*}}\right)$, so $\hat{Y} \supset B\left(z^{*}, \delta^{\prime}\right) \subset \operatorname{clco} \hat{q}^{\tilde{A}, \tilde{a}}$ ( $\tilde{a}$ close to $\left.\tilde{a}^{*}\right)$. See Appendix, Remark V for the next to last inclusion and the implication $(\mathrm{B}) \Rightarrow(\mathrm{A})$.

Now, in the manner required in Corollary I, (34) $\Rightarrow(61)$ (with $\tilde{K}_{*}^{\prime}=2 \xi$, $\left.u^{\prime}, u \in \tilde{U},\left|u^{\prime}-u\right|_{2}=\left|\delta\left(u^{\prime \prime}-u\right)\right|_{2} \leq \delta \tilde{K}_{*}^{\prime}\right),(24)$ and (26) implies continuity of $\tilde{H}$ and $\tilde{a}^{\prime} \rightarrow \tilde{q}^{\tilde{a}^{\prime}, \tilde{a}}$, and (38) implies continuity of $\tilde{a} \rightarrow \tilde{q}^{\tilde{a}^{\prime}, \tilde{a}}$ at $\tilde{a}^{*}$.

\section{Conclusion}

In this paper, necessary conditions for optimal control of diffusions with hard end restrictions on the states have been obtained. The main case considered is the one where the states restricted at the terminal time correspond to differential equations not containing Brownian motions. Brownian motions only occur in differential equations for states unconstrained at the terminal time. A removal of this restriction is discussed in Remark 6.

\section{Acknowledgements}

The author is grateful to a referee for useful comments.

\section{References}

[1] Øksendal, B. (2005) Stochastic Differential Equations. 6th Edition, Springer, New York.

[2] Fleming, W.H. and Rishel, R.W. (1975) Deterministic and Stochastic Control. Springer, New York. https://doi.org/10.1007/978-1-4612-6380-7

[3] Kushner, H.J. (1972) Necessary Conditions for Continuous Parameter Stochastic Optimization Problems. SIAM Journal on Control, 10, 550-565. https://doi.org/10.1137/0310041

[4] Haussmann, U.G. (1986) A Stochastic Maximum Principle for Optimal Control of Diffusions. Pitman Research Notes in Math., No 151, Longman SCI-\&Tech., Harlow.

[5] Peng, S. (1990) A General Stochastic Maximum Principle for Optimal Control Problems. SIAM Journal on Control and Optimization, 28, 966-979. https://doi.org/10.1137/0328054

[6] Yong, J. and Zhou, X.Y. (1999) Stochastic Control. Springer Verlag, New York. https://doi.org/10.1007/978-1-4612-1466-3

[7] Seierstad, A. (2012) Optimal Control of Diffusions with Hard Terminal Constraints. ISRN Applied Mathematics, 2012, Article ID: 729490.

[8] Dunford, N. and Schwartz, J.T. (1967) Linear Operators Part I. Interscience, New York. 
[9] Aubin, J.-P. and Ekeland I. (1984) Applied Nonlinear Analysis. John Wiley \& Sons, New York.

[10] Kallenberg, O. (1997) Foundations of Modern Probability. Springer-Verlag, New York.

[11] Seierstad, A. (1975) An Extension to Banach Space of Pontryagin's Maximum Principle. Journal of Optimization Theory and Applications, 17, 293-335.

https://doi.org/10.1007/BF00933882 


\section{Appendix}

The appendix contains, among other things, a number of wellknown results, included for the convenience of the reader. The first one concerns a result on comparison of solutions. Still $T=1$.

Lemma A. Assume that $\breve{h}(s, z, \omega)$ (an $n_{*}$-vector) and $\sigma_{*}(s, z, \omega)$, (a $n_{*} \times n^{\prime}$ matrix, with columns $\left.\sigma_{*}^{j}, j=1, \cdots, n^{\prime}\right)$ are Lipschitz continuous in $z \in \mathbb{R}^{n_{*}}$ with rank $\breve{K}$ and progressively measurable in $(s, \omega)$. Assume that six progressively measurable functions

$\breve{z}(s, \omega), \alpha(s, \omega), \alpha^{*}(s, \omega), \breve{y}(s, \omega), \beta(s, \omega)$ and $\beta^{*}(t, \omega)$ exist $\left(\alpha^{*}, \beta^{*} n \times n^{\prime}\right.$ -matrices), satisfying

$$
\begin{aligned}
\breve{z}(t, \omega)= & \breve{z}_{0}+\int_{0}^{t} \alpha(s, \omega) \mathrm{d} s+\int_{0}^{t} \breve{h}(s, \breve{z}(s, \omega), \omega) \mathrm{d} s \\
& +\int_{0}^{t} \alpha^{*}(s, \omega) \mathrm{d} B_{s}+\int_{0}^{t} \sigma_{*}(s, \breve{z}(s, \omega), \omega) \mathrm{d} B_{s}
\end{aligned}
$$

and

$$
\begin{aligned}
\breve{y}(t, \omega)= & \breve{y}_{0}+\int_{0}^{t} \beta(s, \omega) \mathrm{d} s+\int_{0}^{t} \breve{h}(s, \breve{y}(s, \omega), \omega) \mathrm{d} s \\
& +\int_{0}^{t} \beta^{*}(s, \omega) \mathrm{d} B_{s}+\int_{0}^{t} \sigma_{*}(s, \breve{y}(s, \omega), \omega) \mathrm{d} B_{s},
\end{aligned}
$$

where $\breve{z}_{0}, \breve{y}_{0} \in L_{2}\left(\Omega, \Phi_{0}, \mathbb{R}^{n}\right)$. We assume that the eight integrands belong to $L_{2}(J \times \Omega)$-spaces. Then, for some constant $\breve{D}$,

$$
\begin{aligned}
& \sup _{s \leq t}|\breve{z}(s)-\breve{y}(s)|_{2} \\
& \leq \breve{D}\left[\left|\breve{y}_{0}-\breve{z}_{0}\right|_{2}+\sup _{s \leq t}\left|\int_{0}^{s} \alpha \mathrm{d} \breve{s}\right|_{2}+\sup _{s \leq t}\left|\int_{0}^{s} \beta \mathrm{d} \breve{s}\right|_{2}\right. \\
& \left.+\sum_{j}\left(E \int_{0}^{t}\left|\alpha_{j}^{*}\right|^{2} \mathrm{~d} \breve{s}\right)^{1 / 2}+\sum_{j}\left(E \int_{0}^{t}\left|\beta_{j}^{*}\right|^{2} \mathrm{~d} \breve{s}\right)^{1 / 2}\right]
\end{aligned}
$$

(applied to matrices the index $j$ indicates columns), and for some constant $\breve{D}^{*}$,

$$
\begin{aligned}
& \left|\left(\sup _{s \leq t}|\breve{z}(s)-\breve{y}(s)|\right)\right|_{2} \\
& \leq \breve{D}^{*}\left[\left|\breve{y}_{0}-\breve{z}_{0}\right|_{2}+\left|\left(\sup _{s \leq t}\left|\int_{0}^{s} \alpha \mathrm{d} \breve{s}\right|\right)\right|_{2}+\left|\left(\sup _{s \leq t}\left|\int_{0}^{s} \beta \mathrm{d} \breve{s}\right|\right)\right|_{2}\right. \\
& \left.+\sum_{j}\left(E \int_{0}^{t}\left|\alpha_{j}^{*}\right|^{2} \mathrm{~d} \breve{s}\right)^{1 / 2}+\sum_{j}\left(E \int_{0}^{t}\left|\beta_{j}^{*}\right|^{2} \mathrm{~d} \breve{s}\right)^{1 / 2}\right] \\
& \leq \breve{D}^{*}\left[\left|\breve{y}_{0}-\breve{z}_{0}\right|_{2}+\left(E \int_{0}^{t}|\alpha|^{2} \mathrm{~d} \breve{s}\right)^{1 / 2}+\left(E \int_{0}^{t}|\beta|^{2} \mathrm{~d} \breve{s}\right)^{1 / 2}\right. \\
& \left.+\sum_{j}\left(E \int_{0}^{t}\left|\alpha_{j}^{*}\right|^{2} \mathrm{~d} \breve{s}\right)^{1 / 2}+\sum_{j}\left(E \int_{0}^{t}\left|\beta_{j}^{*}\right|^{2} \mathrm{~d} \breve{s}\right)^{1 / 2}\right],
\end{aligned}
$$

$\breve{D}$ and $\breve{D}^{*}$ only dependent on $\breve{K}$.

Proof of (57). We shall use a shorthand notation. Using the algebraic inequality $\left(\sum_{j=1}^{N}\left|a_{j}\right|\right)^{2} \leq N \sum_{j} a_{j}^{2}$, then for some positive constant $k$, 


$$
\begin{aligned}
\phi(t):= & |\breve{y}(t)-\breve{z}(t)|^{2} \\
= & \mid \breve{y}_{0}-\breve{z}_{0}+\int_{0}^{t} \breve{h}(s, \breve{y}) \mathrm{d} s+\sum_{j} \int_{0}^{t} \sigma_{*}^{j}(s, \breve{y}) \mathrm{d} B_{s}^{j} \\
& +\int_{0}^{t} \beta \mathrm{d} s-\int_{0}^{t} \alpha \mathrm{d} s-\int_{0}^{t} \breve{h}(s, \breve{z}) \mathrm{d} s \\
& -\sum_{j} \int_{0}^{t} \sigma_{*}^{j}(s, \breve{z}) \mathrm{d} B_{s}^{j}-\sum_{j} \int_{0}^{t} \alpha_{j}^{*} \mathrm{~d} B_{s}^{j}+\left.\int_{0}^{t} \beta_{j}^{*} \mathrm{~d} B_{s}^{j}\right|^{2} \\
\leq & k\left|\breve{y}_{0}-\breve{z}_{0}\right|^{2}+k\left|\int_{0}^{t}(\breve{h}(s, \breve{y})-\breve{h}(s, \breve{z})) \mathrm{d} s\right|^{2} \\
& +k \sum_{j}\left|\int_{0}^{t}\left\{\sigma_{*}^{j}(s, \breve{y})-\sigma_{*}^{j}(s, \breve{z})\right\} \mathrm{d} B_{s}^{j}\right|^{2}+k\left|\int_{0}^{t} \alpha \mathrm{d} s\right|^{2} \\
& +k\left|\int_{0}^{t} \beta \mathrm{d} s\right|^{2}+k \sum_{j}\left|\int_{0}^{t} \alpha_{j}^{*} \mathrm{~d} B_{s}^{j}\right|^{2}+k \sum_{j}\left|\int_{0}^{t} \beta_{j}^{*} \mathrm{~d} B_{s}^{j}\right|^{2}
\end{aligned}
$$

The Burkholder-Davis-Gundy inequality yields, for a "universal" constant $\tilde{K}$, that $\operatorname{Esup}_{s \leq t}\left|\int_{0}^{s}\left(\sigma_{*}^{j}(\breve{s}, \breve{y})-\sigma_{*}^{j}(\breve{s}, \breve{z})\right) \mathrm{d} B_{\breve{s}}^{j}\right|^{2} \leq \tilde{K} \int_{0}^{t} E\left|\sigma_{*}^{j}(\breve{s}, \breve{y})-\sigma_{*}^{j}(\breve{s}, \breve{z})\right|^{2} \mathrm{~d} \breve{s}$. Similar inequalities hold for the other terms involving $B^{j}$. Hence (using also Jensen's inequality) we get

$$
\begin{aligned}
& \psi(t):=E\left(\sup _{s \leq t} \phi(s)\right) \\
& \leq k E\left|\breve{y}_{0}-\breve{z}_{0}\right|^{2}+k E \sup _{s \leq t} s \int_{0}^{s}|\breve{h}(\breve{s}, \breve{y})-\breve{h}(\breve{s}, \breve{z})|^{2} \mathrm{~d} \breve{s} \\
& +k E \sum_{j} \sup _{s \leq t}\left|\int_{0}^{s}\left(\sigma_{*}^{j}(\breve{s}, \breve{y})-\sigma_{*}^{j}(\breve{s}, \breve{z})\right) \mathrm{d} B_{\breve{s}}^{j}\right|^{2} \\
& +k E \sup _{s \leq t}\left|\int_{0}^{s} \alpha \mathrm{d} \breve{s}\right|^{2}+k E \sup _{s \leq t}\left|\int_{0}^{s} \beta \mathrm{d} \breve{s}\right|^{2} \\
& +k E \sum_{j} \sup _{s \leq t}\left|\int_{0}^{s} \alpha_{j}^{*} \mathrm{~d} B_{s}^{j}\right|^{2}+k E \sum_{j} \sup _{s \leq t}\left|\int_{0}^{s} \beta_{j}^{*} \mathrm{~d} B_{s}^{j}\right|^{2} \\
& \leq k E\left|\breve{y}_{0}-\breve{z}_{0}\right|^{2}+k \int_{0}^{t} E|\breve{h}(\breve{s}, \breve{y})-\breve{h}(\breve{s}, \breve{z})|^{2} \mathrm{~d} \breve{s} \\
& +k \tilde{K} \sum_{j} \int_{0}^{t} E\left|\sigma_{*}^{j}(\breve{s}, \breve{y})-\sigma_{*}^{j}(s, \breve{z})\right|^{2} \mathrm{~d} \breve{s} \\
& +k E \sup _{s \leq t}\left|\int_{0}^{s} \alpha \mathrm{d} \breve{s}\right|^{2}+k E \sup _{s \leq t}\left|\int_{0}^{s} \beta \mathrm{d} \breve{s}\right|^{2} \\
& +k \tilde{K} \sum_{j} E \int_{0}^{t}\left|\alpha_{j}^{*}\right|^{2} \mathrm{~d} \breve{s}+k \tilde{K} \sum_{j} E \int_{0}^{t}\left|\beta_{j}^{*}\right|^{2} \mathrm{~d} \breve{s} \\
& \leq k E\left|\breve{y}_{0}-\breve{z}_{0}\right|^{2}+k \int_{0}^{t} E \breve{K}^{2}|\breve{y}-\breve{z}|^{2} \mathrm{~d} \breve{s}+k \tilde{K} n^{\prime} \int_{0}^{t} E \breve{K}^{2}|\breve{y}-\breve{z}|^{2} \mathrm{~d} \breve{s} \\
& +k E\left(\sup _{s \leq t}\left|\int_{0}^{s} \alpha \mathrm{d} \breve{s}\right|\right)^{2}+k E\left(\sup _{s \leq t}\left|\int_{0}^{s} \beta \mathrm{d} \breve{s}\right|\right)^{2} \\
& +k \tilde{K} \sum_{j} E \int_{0}^{t}\left|\alpha_{j}^{*}\right|^{2} \mathrm{~d} \breve{s}+k \tilde{K} \sum_{j} E \int_{0}^{t}\left|\beta_{j}^{*}\right|^{2} \mathrm{~d} s \\
& \leq k E\left|\breve{y}_{0}-\breve{z}_{0}\right|^{2}+k \breve{K}^{2} \int_{0}^{t} \psi(\breve{s}) \mathrm{d} \breve{s}+k \tilde{K} \breve{K}^{2} n^{\prime} \int_{0}^{t} \psi(\breve{s}) \mathrm{d} \breve{s} \\
& +k E\left(\sup _{s \leq t}\left|\int_{0}^{s} \alpha \mathrm{d} \breve{s}\right|\right)^{2}+k E\left(\sup _{s \leq t}\left|\int_{0}^{s} \beta \mathrm{d} \breve{s}\right|\right)^{2} \\
& +k \tilde{K} \sum_{j} E \int_{0}^{t}\left|\alpha_{j}^{*}\right|^{2} \mathrm{~d} \breve{s}+k \tilde{K} \sum_{j} E \int_{0}^{t}\left|\beta_{j}^{*}\right|^{2} \mathrm{~d} s .
\end{aligned}
$$

Note that, by Gronwall's inequality, for any functions $w(t), v(t)$, if $0 \leq w(t) \leq v(t)+\int_{0}^{t} K w(s) \mathrm{d} s$, and $v(t)$ is increasing, then $w(t) \leq v(t)\left(1+\mathrm{e}^{K t}\right)$. 
Hence, for $\breve{K}^{2}:=k\left(1+\mathrm{e}^{k \breve{K}^{2}\left(1+\tilde{K} n^{\prime}\right)}\right)$,

$$
\begin{aligned}
\psi(t) \leq & \breve{K}^{2}\left[E\left|\breve{y}_{0}-\breve{z}_{0}\right|^{2}+E\left(\sup _{s \leq t}\left|\int_{0}^{s} \alpha \mathrm{d} \breve{s}\right|\right)^{2}+E\left(\sup _{s \leq t}\left|\int_{0}^{s} \beta \mathrm{d} \breve{s}\right|\right)^{2}\right. \\
& \left.+\tilde{K} \sum_{j} E \int_{0}^{t}\left|\alpha_{j}^{*}\right|^{2} \mathrm{~d} \breve{s}+\tilde{K} \sum_{j} E \int_{0}^{t}\left|\beta_{j}^{*}\right|^{2} \mathrm{~d} s\right] .
\end{aligned}
$$

Using the fact that the square root of a sum of positive numbers is $\leq$ the sum of square roots of the numbers, we get

$$
\begin{aligned}
& \left|\left(\sup _{s \leq t}|y(s)-z(s)|\right)\right|_{2} \\
& \leq \breve{K}\left[\left|\breve{y}_{0}-\breve{z}_{0}\right|_{2}+\left|\left(\sup _{s \leq t}\left|\int_{0}^{s} \alpha \mathrm{d} \widetilde{s}\right|\right)\right|_{2}+\left|\left(\sup _{s \leq t}\left|\int_{0}^{s} \beta \mathrm{d} \breve{s}\right|\right)\right|_{2}\right. \\
& \left.\quad+\sum_{j}\left(\tilde{K} \int_{0}^{t} E\left|\alpha_{j}^{*}\right|^{2} \mathrm{~d} \widetilde{s}\right)^{1 / 2}+\sum_{j}\left(\tilde{K} \int_{0}^{t} E\left|\beta_{j}^{*}\right|^{2} \mathrm{~d} s\right)^{1 / 2}\right] .
\end{aligned}
$$

Note that $\sup _{s \leq t}\left|\int_{0}^{s} \alpha \mathrm{d} \breve{s}\right| \leq \sup _{s \leq t} \int_{0}^{s}|\alpha| \mathrm{d} \breve{s} \leq \int_{0}^{t}|\alpha| \mathrm{d} \breve{s}$, and that $\left|\int_{0}^{t}\right| \alpha|\mathrm{d} \breve{s}|_{2} \leq \int_{0}^{t}|\alpha|_{2} \mathrm{~d} \breve{s}$. Using this for the term containing $\alpha$, and a similar argument for the term containing $\beta$, then (57) follows.

Proof of (56).

Using Ito's isometry,

$$
\begin{aligned}
E\left|\int_{0}^{t} \sigma_{*}^{j}(s, \breve{y})-\sigma_{*}^{j}(s, \breve{z}) \mathrm{d} B_{s}^{j}\right|^{2} & \leq E \int_{0}^{t}\left|\sigma_{*}^{j}(s, \breve{y})-\sigma_{*}^{j}(s, \breve{z})\right|^{2} \mathrm{~d} s, \\
E\left|\int_{0}^{t} \alpha_{j}^{*} \mathrm{~d} B_{s}^{j}\right|^{2} & =E \int_{0}^{t}\left|\alpha_{j}^{*}\right|^{2} \mathrm{~d} s, \\
E\left|\int_{0}^{t} \beta_{j}^{*} \mathrm{~d} B_{s}^{j}\right|^{2} & =E \int_{0}^{t}\left|\beta_{j}^{*}\right|^{2} \mathrm{~d} s .
\end{aligned}
$$

Then, again using $\left(\sum_{j=1}^{N}\left|a_{j}\right|\right)^{2} \leq N \sum_{j} a_{j}^{2}$ and Jensen's inequality, for some positive constant $k$,

$$
\begin{aligned}
& \gamma(t):=\sup _{s \leq t} E \phi(s) \\
& \leq k E\left|\breve{y}_{0}-\breve{z}_{0}\right|^{2}+k \sup _{s \leq t} E s \int_{0}^{s}|\breve{h}(\breve{y})-\breve{h}(\breve{z})|^{2} \mathrm{~d} \breve{s} \\
& +k \sum_{j} \sup _{s \leq t} E\left|\int_{0}^{s}\left(\sigma_{*}^{j}(\breve{y})-\sigma_{*}^{j}(\breve{z})\right) \mathrm{d} B_{\breve{s}}^{j}\right|^{2} \\
& +k \sup _{s \leq t} E\left|\int_{0}^{s} \alpha \mathrm{d} \breve{s}\right|^{2}+k \sup _{s \leq t} E\left|\int_{0}^{s} \beta \mathrm{d} \breve{s}\right|^{2} \\
& +k \sum_{j} \sup _{s \leq t} E\left|\int_{0}^{s} \alpha_{j}^{*} \mathrm{~d}_{\breve{s}}^{j}\right|^{2}+k \sum_{j} \sup _{s \leq t} E\left|\int_{0}^{s} \beta_{j}^{*} \mathrm{~d} B_{\breve{s}}^{j}\right|^{2} \\
& \leq k E\left|\breve{y}_{0}-\breve{z}_{0}\right|^{2}+k \sup _{s \leq t} \int_{0}^{s} E|\breve{h}(\breve{y})-\breve{h}(\breve{z})|^{2} \mathrm{~d} \breve{s} \\
& +k \sum_{j} \sup _{s \leq t} \int_{0}^{s} E\left|\sigma_{*}^{j}(\breve{y})-\sigma_{*}^{j}(\breve{z})\right|^{2} \mathrm{~d} \breve{s} \\
& +k \sup _{s \leq t} E\left|\int_{0}^{s} \alpha \mathrm{d} \breve{s}\right|^{2}+k \sup _{s \leq t} E\left|\int_{0}^{s} \beta \mathrm{d} \breve{s}\right|^{2} \\
& +k \sum_{j} \sup _{s \leq t} E \int_{0}^{s}\left|\alpha_{j}^{*}\right|^{2} \mathrm{~d} \breve{s}+k \sum_{j} \sup _{s \leq t} E \int_{0}^{s}\left|\beta_{j}^{*}\right|^{2} \mathrm{~d} s
\end{aligned}
$$




$$
\begin{aligned}
\leq & k E\left|\breve{y}_{0}-\breve{z}_{0}\right|^{2}+k \int_{0}^{t} E \breve{K}^{2}|\breve{y}-\breve{z}|^{2} \mathrm{~d} \breve{s}+k n^{\prime} \int_{0}^{t} E \breve{K}^{2}|\breve{y}-\breve{z}|^{2} \mathrm{~d} \breve{s} \\
& +k \sup _{s \leq t} E\left|\int_{0}^{s} \alpha \mathrm{d} \breve{s}\right|^{2}+k \sup _{s \leq t} E\left|\int_{0}^{s} \beta \mathrm{d} \breve{s}\right|^{2} \\
& +k \sum_{j} E \int_{0}^{t}\left|\alpha_{j}^{*}\right|^{2} \mathrm{~d} \breve{s}+k \sum_{j} E \int_{0}^{t}\left|\beta_{j}^{*}\right|^{2} \mathrm{~d} s \\
\leq & k E\left|\breve{y}_{0}-\breve{z}_{0}\right|^{2}+k \breve{K}^{2} \int_{0}^{t} \gamma(\breve{s}) \mathrm{d} \breve{s}+k \breve{K}^{2} n^{\prime} \int_{0}^{t} \gamma(\breve{s}) \mathrm{d} \breve{s} \\
& +k\left(\sup _{s \leq t} E\left|\int_{0}^{s} \alpha \mathrm{d} \breve{s}\right|\right)^{2}+k\left(\sup _{s \leq t} E\left|\int_{0}^{s} \beta \mathrm{d} \breve{s}\right|\right)^{2} \\
& +k \sum_{j} E \int_{0}^{t}\left|\alpha_{j}^{*}\right|^{2} \mathrm{~d} \breve{s}+k \sum_{j} E \int_{0}^{t}\left|\beta_{j}^{*}\right|^{2} \mathrm{~d} s .
\end{aligned}
$$

Thus, for $\breve{K}^{*^{2}}=k\left(1+\mathrm{e}^{k \breve{K}^{2}\left(1+n^{\prime}\right)}\right)$,

$$
\begin{aligned}
& \sup _{s \leq t} E|\breve{y}(t)-\breve{z}(t)|^{2} \\
& \leq \breve{K}^{* 2}\left[E\left|\breve{y}_{0}-\breve{z}_{0}\right|^{2}+\sup _{s \leq t} E\left|\int_{0}^{s} \alpha \mathrm{d} \breve{s}\right|^{2}+\sup _{s \leq t} E\left|\int_{0}^{s} \beta \mathrm{d} \breve{s}\right|^{2}\right. \\
& \left.+E \sum_{j} \int_{0}^{t}\left|\alpha_{j}^{*}\right|^{2} \mathrm{~d} \breve{s}+E \sum_{j} \int_{0}^{t}\left|\beta_{j}^{*}\right|^{2} \mathrm{~d} s\right],
\end{aligned}
$$

so (56) follows.

Simple results om Gâteaux derivatives appear in the next two lemmas.

Lemma B. Let $\breve{\sigma}(t, z): J \times \mathbb{R}^{n_{*}} \rightarrow \mathbb{R}^{n_{* *}}$ be continuously differentiable in $z$ for each $t$, and have one-sided limits with respect to $t$, and assume $\left|\breve{\sigma}_{z}(t, z)\right| \leq M_{*}$ for all $t, z$. For each $z(.,.) \in L_{2}^{\text {prog }}\left(J \times \Omega, \mathbb{R}^{n_{*}}\right)$, for each "direction" $\breve{z}(.,.) \in L_{2}^{\text {prog }}\left(J \times \Omega, \mathbb{R}^{n_{*}}\right)$, and for each $t, \quad z^{\prime}(t, \omega) \rightarrow \int_{0}^{t} \breve{\sigma}\left(s, z^{\prime}(s, \omega)\right) \mathrm{d} B_{s}$ $\left(z^{\prime}(.,.) \in L_{2}^{\text {prog }}\left(J \times \Omega, \mathbb{R}^{n_{*}}\right)\right)$, has, in the norm $|\cdot|_{2}$, a bounded linear Gâteaux derivative at $z(.,$.$) in direction \breve{z}(.,$.$) , which equals$ $\int_{0}^{t} \breve{\sigma}_{z}(s, z(s, \omega)) \breve{z}(s, \omega) \mathrm{d} B_{s}$. The derivative is uniform in $t$.

Proof. By Ito's isometry,

$$
\begin{aligned}
& E \mid \int_{0}^{t} \delta^{-1}[\breve{\sigma}(s, z(s, \omega)+\delta \breve{z}(s, \omega))-\breve{\sigma}(s, z(s, \omega)) \\
& \left.-\breve{\sigma}_{z}(s, z(s, \omega)) \delta \breve{z}(s, \omega)\right]\left.\mathrm{d} B_{s}\right|^{2} \\
& =E \int_{0}^{t} \mid \delta^{-1}[\breve{\sigma}(s, z(s, \omega)+\delta \breve{z}(s, \omega))-\breve{\sigma}(s, z(s, \omega))] \\
& \left.-\left.\breve{\sigma}_{z}(s, z(s, \omega)) \breve{z}(s, \omega)\right|^{2} \mathrm{~d} s\right) \\
& =: \beta^{* *}(t) \leq \beta^{* *}(1)
\end{aligned}
$$

When $\delta \rightarrow 0$, the term in curly brackets converges to zero for each $(s, \omega)$ and is smaller than the $L_{1}$-function $\left(2 M_{*}\right)^{2}|\breve{z}(s, \omega)|^{2}$. Hence, Lebesgue's dominated convergence theorem gives that $\beta^{* *}(1) \rightarrow 0$ when $\delta \rightarrow 0$.

Lemma C. Let $\breve{f}(t, z): J \times \mathbb{R}^{n_{*}} \rightarrow \mathbb{R}^{n_{* *}}$ be continuously differentiable in $z$ for each $t$, and have one-sided limits with respect to $t$, and assume $\left|\breve{f}_{z}(t, z)\right| \leq M_{*}$ for all $t, z$. For each $z(.,.) \in L_{2}^{\text {prog }}(J \times \Omega, Z)$, for each "direction" $\breve{z}(.,.) \in L_{2}^{\text {prog }}(J \times \Omega, X)$, and for each $t, \quad z^{\prime}(t, \omega) \rightarrow \int_{0}^{t} \breve{f}\left(s, z^{\prime}(s, \omega)\right) \mathrm{d} s$ $\left(z^{\prime}(.,.) \in L_{2}^{\text {prog }}(J \times \Omega, X)\right)$, has, in the norm $|\cdot|_{2}$, a bounded linear Gâteaux 
derivative at $z(.,$.$) in direction \breve{z}(.,$.$) , which in equals$ $\int_{0}^{t} \breve{f}_{x}(s, z(s, \omega)) \breve{z}(s, \omega) \mathrm{d} s$. The derivative is uniform in $t$.

Proof. Define

$$
\begin{aligned}
\beta^{*}(t):= & E \mid \int_{0}^{t} \delta^{-1}\{\breve{f}(s, z(s, \omega)+\delta \breve{z}(s, \omega))-\breve{f}(s, z(s, u)) \\
& \left.-\breve{f}_{z}(s, z(s, \omega)) \delta \breve{z}(s, \omega)\right\}\left.\mathrm{d} s\right|^{2}, \\
\beta^{* *}(t):= & E \int_{0}^{t} \mid \delta^{-1}\{\breve{f}(s, z(s, \omega)+\delta \breve{z}(s, \omega))-\breve{f}(s, z(s, u)) \\
& \left.-f_{x}(s, z(s, \omega)) \delta \breve{z}(s, \omega)\right\}\left.\right|^{2} \mathrm{~d} s .
\end{aligned}
$$

Jensen's inequality yields the inequality $\beta^{*}(t) \leq \beta^{* *}(t)$. The remaining arguments are as in the preceding proof, they yield

$0 \leq \beta^{*}(t) \leq \beta^{* *}(t) \leq \beta^{* *}(1) \rightarrow 0$ when $\delta \rightarrow 0$.

Below, on product spaces, maximum norms (= maximum of norms) and maximum metrics are used. In the sequel, the following entities are used:

$Y$ is a normed space, $A$ is a complete pseudometric space with pseudo-metric $\rho$, and $a^{*}$ is a given element in $A$. The function $H(a)$ from $A$ into $Y$ is continuous.

Theorem D. (Attainability) Let the entities in (58) be given. Let positive numbers $K, \hat{\mu}, \mu^{\prime}, \mu, \mu \in(0,1)$, and an element $\hat{z}^{*}$ in $Y$ be given. Assume that the following properties hold for all $a \in \operatorname{cl} B\left(a^{*}, \hat{\mu}\right):$ For all $\hat{v} \in Y$ with $\left|\hat{v}-\hat{z}^{*}\right|=\mu^{\prime}$, for all $r>0$, a pair $\left(a^{\prime}, \delta\right) \in A \times(0, r]$ exists, such that

$$
\left|H\left(a^{\prime}\right)-H(a)-\delta \hat{v}\right| \leq(1-\mu) \delta \mu^{\prime}|\hat{v}| /\left(\left|\hat{z}^{*}\right|+\mu^{\prime}\right) \text { and } \rho\left(a^{\prime}, a\right) \leq \delta K|\hat{v}| \text {. }
$$

Then, for all $z \in \operatorname{cl} B\left(H\left(a^{*}\right), \mu \mu^{\prime} \hat{\mu} / 4 K\left(\left|\hat{z}^{*}\right|+\mu^{\prime}\right)\right)$, there exists a pair $(a, \alpha) \in \operatorname{cl} B\left(a^{*}, \hat{\mu} \gamma / 2\right) \times\left[0, \hat{\mu} \gamma / 2 K\left(\left|\hat{z}^{*}\right|+\mu^{\prime}\right)\right]$, such that $z+\alpha \hat{z}^{*}=H(a)$, where $\gamma:=4 K\left(\left|\hat{z}^{*}\right|+\mu^{\prime}\right)\left|H\left(a^{*}\right)^{-}-z\right| / \mu \mu^{\prime} \hat{\mu} \leq 1$.

Corollary E. Assume that $w:=\inf \left\{|\hat{v}|:\left|\hat{v}-\hat{z}^{*}\right|=\mu^{\prime}\right\}>0$. Then, in (59), evidently $\rho\left(a^{\prime}, a\right) \leq \delta K|\hat{v}|$ can be replaced by the stronger inequality $\rho\left(a^{\prime}, a\right) \leq \delta K w$.

(On the other hand, when $w>0$, then $\rho\left(a^{\prime}, a\right) \leq \delta K|\hat{v}| \Rightarrow \rho\left(a^{\prime}, a\right) \leq \delta K^{\prime} w$ for $K^{\prime}=\left(\left|\hat{z}^{*}\right|+\mu^{\prime}\right) K / w$. $)$

Central ideas in the proof of Theorem D stem from the proof of the multifunction inverse function theorem Theorem 4, p. 431, in [9].

Proof of Theorem D. The property (59) also holds for $\hat{v}$ in the set $B^{*}:=\left\{\lambda \tilde{v}: \lambda>0, \tilde{v} \in Y,\left|\tilde{v}-\hat{z}^{*}\right|=\mu^{\prime}\right\}$. To see this, let $\hat{v}^{\prime} \in B^{*}$ and let $r>0$. Then $\hat{v}^{\prime}=\lambda \hat{v}$ for some $\lambda>0$, some $\hat{v}$ such that $\left|\hat{v}-\hat{z}^{*}\right|=\mu^{\prime}$. Now, for all $a \in \operatorname{cl} B\left(a^{*}, \hat{\mu}\right)$, there exists a pair $\left(a^{\prime}, \delta\right), 0<\delta \leq r \lambda$, such that the inequalities in (59) hold. From these inequalities, for $\delta^{\prime}:=\delta / \lambda \in(0, r]$, using $\delta^{\prime} \hat{v}^{\prime}=\delta \hat{v}$, it follows that $\left|H\left(a^{\prime}\right)-H(a)-\delta^{\prime} \hat{v}^{\prime}\right| \leq(1-\mu) \delta^{\prime} \mu^{\prime}\left|\hat{v}^{\prime}\right| /\left(\left|\hat{z}^{*}\right|+\mu^{\prime}\right)$ and $\rho\left(a^{\prime}, a\right) \leq \delta^{\prime} K\left|\hat{v}^{\prime}\right|$. Hence, (59) holds for $\hat{v}^{\prime} \in B^{*}$.

Below, write $\left|\hat{z}^{*}\right|+\mu^{\prime}=: \kappa$. The following lemma is needed in the proof:

Lemma F. Let $z \in \operatorname{cl} B\left(H\left(a^{*}\right), \mu \mu^{\prime} \hat{\mu} / 4 K \kappa\right)$. Assume that the pair 


$$
\begin{aligned}
& \left(a_{1}, \lambda_{1}\right) \in \operatorname{clB}\left(a^{*}, \hat{\mu} / 2\right) \times[-\hat{\mu} / 2 K \kappa, 0] \text { minimizes } \\
& \quad(a, \lambda) \rightarrow\left|H(a)+\lambda \hat{z}^{*}-z\right|+\left(\mu \mu^{\prime} / 2 K \kappa\right) \max \left\{\rho\left(a, a_{1}\right),\left|\lambda-\lambda_{1}\right| K \kappa\right\}
\end{aligned}
$$

in $\left.\operatorname{cl} B\left(a^{*}, \hat{\mu}\right)\right) \times[-\hat{\mu} / K \kappa, 0]$. Then $\left|H\left(a_{1}\right)+\lambda_{1} \hat{z}^{*}-z\right|=0$.

Proof of Lemma F. By contradiction, assume $|\hat{z}|>0, \hat{z}:=H\left(a_{1}\right)+\lambda_{1} \hat{z}^{*}-z$. The vector $\hat{v}:=\hat{z}^{*}-\mu^{\prime} \hat{z}|| \hat{z} \mid$ satisfies $\left|\hat{v}-\hat{z}^{*}\right|=\mu^{\prime}$, so $|\hat{z}| \hat{v}=|\hat{z}| \hat{z}^{*}-\mu^{\prime} \hat{z}$ belongs to $B^{*}$. Hence, by the extended property (59), there exist an $a^{\prime} \in A$ and a $\delta \leq \hat{\mu} /(2 K \kappa|\hat{z}|), \quad \delta \in\left(0,1 / \mu^{\prime}\right]$, such that

$$
\begin{aligned}
& \left|H\left(a^{\prime}\right)-H\left(a_{1}\right)-\delta\left(|\hat{z}| \hat{z}^{*}-\mu^{\prime} \hat{z}\right)\right| \\
& \leq(1-\mu) \delta \mu^{\prime}\left|\left(|\hat{z}| \hat{z}^{*}-\mu^{\prime} \hat{z}\right)\right| / \kappa \leq(1-\mu) \delta \mu^{\prime} /|\hat{z}| .
\end{aligned}
$$

Moreover, $\rho\left(a^{\prime}, a_{1}\right) \leq \delta K\left|\left(|\hat{z}| \hat{z}^{*}-\mu^{\prime} \hat{z}\right)\right| \leq \hat{\mu} / 2$, (use the first inequality for $\delta$ ), which implies $\quad a^{\prime} \in \operatorname{cl} B\left(a^{*}, \hat{\mu}\right) \quad$ Define $\quad \lambda^{\prime}=\lambda_{1}-\delta|\hat{z}| \in[-\hat{\mu} / K \kappa, 0]$ $(\delta|\hat{z}| \leq \hat{\mu} / 2 K \kappa)$. Then, using (60), $\delta \mu^{\prime} \leq 1$, and the definition of $\hat{z}$, we get

$$
\begin{aligned}
& \left|H\left(a^{\prime}\right)+\lambda^{\prime} \hat{z}^{*}-z\right|=\left|-z+H\left(a_{1}\right)+H\left(a^{\prime}\right)-H\left(a_{1}\right)+\lambda_{1} \hat{z}^{*}-\delta\right| \hat{z}\left|\hat{z}^{*}\right| \\
& \leq\left|-z+H\left(a_{1}\right)+\delta\left(|\hat{z}| \hat{z}^{*}-\mu^{\prime} \hat{z}\right)+\lambda_{1} \hat{z}^{*}-\delta\right| \hat{z}\left|\hat{z}^{*}\right|+(1-\mu) \delta \mu^{\prime}|\hat{z}| \\
& =\left|-z+H\left(a_{1}\right)+\lambda_{1} \hat{z}^{*}-\delta \mu^{\prime} \hat{z}\right|+(1-\mu) \delta \mu^{\prime}|\hat{z}| \\
& =\left|\hat{z}-\delta \mu^{\prime} \hat{z}\right|+(1-\mu) \delta \mu^{\prime}|\hat{z}| \\
& \leq\left(1-\delta \mu^{\prime}\right)|\hat{z}|+(1-\mu) \delta \mu^{\prime}|\hat{z}|=\left(1-\mu \delta \mu^{\prime}\right)|\hat{z}| .
\end{aligned}
$$

Using $\left|H\left(a^{\prime}\right)+\lambda^{\prime} \hat{z}^{*}-z\right| \leq\left(1-\mu \delta \mu^{\prime}\right)|\hat{z}|$ and $\lambda^{\prime}-\lambda_{1}=-\delta|\hat{z}|$ yields

$$
\begin{aligned}
& \left|-z+H\left(a^{\prime}\right)+\lambda^{\prime} z^{*}\right|+\left(\mu \mu^{\prime} / 2 K \kappa\right) \max \left\{\rho\left(a^{\prime}, a_{1}\right),\left|\lambda^{\prime}-\lambda_{1}\right| K \kappa\right\} \\
& \leq\left(1-\mu \delta \mu^{\prime}\right)|\hat{z}|+\left(\mu \mu^{\prime} / 2 K \kappa\right) \max \left\{\delta K\left|\left(|\hat{z}| \hat{z}^{*}-\mu^{\prime} \hat{z}\right)\right|, \delta|\hat{z}| K \kappa\right\} \\
& \leq\left(1-\mu \delta \mu^{\prime}\right)|\hat{z}|+\mu \delta \mu^{\prime}|\hat{z}| / 2<|\hat{z}| \\
& =\left|H\left(a_{1}\right)+\lambda_{1} \hat{z}^{*}-z\right|+\left(\mu \mu^{\prime} / 2 K \kappa\right) \max \left\{\rho\left(a_{1}, a_{1}\right),\left|\lambda_{1}-\lambda_{1}\right| K \kappa\right\},
\end{aligned}
$$

a contradiction of the optimality of $\left(a_{1}, \lambda_{1}\right)$.

Continued proof of the theorem. Let $z \in \operatorname{cl} B\left(H\left(a^{*}\right), \mu \mu^{\prime} \hat{\mu} / 4 K \kappa\right)$, let $\gamma$ be as in the conclusion of the theorem, and let $\phi(a, \lambda):=\left|H(a)+\lambda \hat{z}^{*}-z\right|$. Note that $\phi\left(a^{*}, 0\right):=\left|H\left(a^{*}\right)-z\right| \leq \gamma \mu \mu^{\prime} \hat{\mu} / 4 K \kappa$. Let the distance between $(a, \lambda)$ and $\left(a^{\prime \prime}, \lambda^{\prime \prime}\right)$ be $\left(\mu \mu^{\prime} / 2 K \kappa\right) \max \left\{\rho\left(a, a^{\prime \prime}\right),\left|\lambda-\lambda^{\prime \prime}\right| K \kappa\right\} \quad$ in the complete space $\operatorname{clB}\left(a^{*}, \hat{\mu}\right) \times[-\hat{\mu} / K \kappa, 0]$. By Aubin and Ekeland (1984, Theorem 1, p. 255), (Ekeland's variational principle), there exists a $\left(a_{1}, \lambda_{1}\right) \in \operatorname{cl} B\left(a^{*}, \hat{\mu}\right) \times[-\hat{\mu} / K \kappa, 0]$ such that

$$
\phi\left(a_{1}, \lambda_{1}\right) \leq \phi(a, \lambda)+\left(\mu \mu^{\prime} / 2 K \kappa\right) \max \left\{\rho\left(a, a_{1}\right),\left|\lambda-\lambda_{1}\right| K \kappa\right\}
$$

for all $(a, \lambda) \in \operatorname{clB}\left(a^{*}, \hat{\mu}\right) \times[-\hat{\mu} / K \kappa, 0]$ and

$$
\begin{aligned}
& \phi\left(a_{1}, \lambda_{1}\right)+\left(\mu \mu^{\prime} / 2 K \kappa\right) \max \left\{\rho\left(a_{1}, a^{*}\right),\left|\lambda_{1}-0\right| K \kappa\right\} \\
& \leq \phi\left(a^{*}, 0\right) \leq \mu \mu^{\prime} \hat{\mu} \gamma / 4 K \kappa,
\end{aligned}
$$

which gives $\rho\left(a_{1}, a^{*}\right) \leq \hat{\mu} \gamma / 2,\left|\lambda_{1}\right| \leq \hat{\mu} \gamma / 2 K \kappa$. By Lemma $\mathrm{F}$, 
$\left|-z+H\left(a_{1}\right)+\lambda_{1} \hat{z}^{*}\right|=0$, so $z+\alpha \hat{z}^{*}=H\left(a_{1}\right)$, for $\alpha=-\lambda_{1} \in[0, \hat{\mu} \gamma / 2 K \kappa]$.

Below, $q^{a^{\prime}, a}$ is a sort of Gâteaux derivative at a of $H($.$) .$

Corollary G. Let $\tilde{\mu}>0, Y$ a normed space, $A$ a complete pseudometric space with metric $\sigma, a^{*}$ a given element in $\mathrm{A}$, and let $H(a): A \rightarrow Y$ be continuous. For each $a \in A$, let $A_{a} \subset A$ be a set dense in $A$. Assume the existence of a function $q^{a^{\prime}, a}$, from $A \times A$ into $Y$ and a positive constant $\tilde{K}^{\prime}$ such that, for each $a \in \operatorname{cl} B\left(a^{*}, \tilde{\mu}\right)$, for all $r>0$, all $\tilde{\varepsilon}>0$, all $a^{+} \in A_{a}$, there exists a pair $\left(a^{\prime}, \delta\right), a^{\prime} \in \operatorname{cl} B\left(a, \tilde{K}^{\prime} \delta\right), \delta \in(0, r]$ such that

$$
\left|H\left(a^{\prime}\right)-H(a)-\delta q^{a^{+}, a}\right| \leq \tilde{\varepsilon} \delta .
$$

Assume also that for all $a \in A$,

$$
\operatorname{coq}^{\mathrm{A}, a} \subset \operatorname{cl} q^{\mathrm{A}, a} .
$$

Assume that $\breve{a} \rightarrow q^{\breve{a}, a}$ is continuous in $A$ for any $a \in A$, and that $a \rightarrow q^{\breve{a}, a}$ is continuous at $a^{*}$ for any $\breve{a} \in A_{a^{*}}$. Assume finally that $b$ is an interior point in $\operatorname{clco} q^{A, a^{*}}$, and that, for some $\tilde{\varepsilon}>0$, some $z^{*} \in Y, B\left(z^{*}, \tilde{\varepsilon}\right) \subset \operatorname{clcoq} q^{A, a}$ for all $a \in \operatorname{cl} B\left(a^{*}, \tilde{\mu}\right)$. Then, for some $\hat{\gamma}>0$ and some $a \in A$, $H\left(a^{*}\right)+\hat{\gamma} b=H(a)$.

Proof. Write $\tilde{Q}^{a}=\operatorname{clcoq}^{A, a}$, and let $B(b, \alpha) \subset \tilde{Q}^{a^{*}}$ for some $\alpha>0$. Then, for some $\kappa>0,-\kappa z^{*} \in B(0, \alpha) \subset \tilde{Q}^{a^{*}}-b$. Define $B_{z}=\operatorname{co}\left\{z, B\left(z^{*}, \tilde{\varepsilon}\right)+b\right\}$. Evidently, $b$ is an interior point in $B_{z}$ if $z=-\kappa z^{*}+b$. Then $b$ is an interior point in $B_{z}$ even if $z=-\kappa z^{*}+b$ is only an approximate equality, in fact there exist positive numbers $\rho^{*}$ and $\xi$ such that $B(b, \xi) \subset B_{z}$ for all $z \in \operatorname{cl} B\left(-\kappa z^{*}+b, \rho^{*}\right)$. Because $-\kappa z^{*} \in \tilde{Q}^{a^{*}}-b$, by (62) there exists a $\tilde{a} \in A$, such that $\left|b-\kappa z^{*}-q^{\tilde{a}, a^{*}}\right|<\rho^{*} / 2$, and we can even assume $\tilde{a} \in A_{a^{*}}$, by density of $A_{a^{*}}$ and continuity of $\breve{a} \rightarrow q^{\breve{a}, a *}$. By the continuity of $a \rightarrow q^{\breve{a}, a}$ in the corollary, for $\beta>0$ small enough, $\left|q^{\tilde{a}, a}-q^{\tilde{a}, a^{*}}\right| \leq \rho^{*} / 2$ for $a \in B\left(a^{*}, \beta\right)$. We assume $\beta \leq \tilde{\mu}, \quad \beta \leq \tilde{K}^{\prime} / 2$. Evidently, $\left|b-\kappa z^{*}-q^{\tilde{a}, a}\right|<\rho^{*}$. Hence, $q^{\tilde{a}, a} \in B\left(-\kappa z^{*}+b, \rho^{*}\right) \quad$ for all $a \in B\left(a^{*}, \beta\right)$. Thus, for $a \in \operatorname{cl} B\left(a^{*}, \beta\right)$, $B(b, \xi) \subset B_{q^{\tilde{a}, a}}:=\operatorname{co}\left\{q^{\tilde{a}, a}, \operatorname{cl} B\left(z^{*}, \tilde{\varepsilon}\right)+b\right\} \subset \operatorname{co}\left\{q^{\tilde{a}, a}, \tilde{Q}^{a}+b\right\} \subset \tilde{Q}^{a}+[0,1] b$, because $q^{\frac{q}{a, a}} \in \tilde{Q}^{a}$ and $\tilde{Q}^{a}$ is convex. Hence, $B(0, \xi) \subset \tilde{Q}^{a}-[0,1] b$, $a \in \operatorname{cl} B\left(a^{*}, \beta\right)$. It follows that if $\hat{v} \in Y,|\hat{v}|=\varsigma=\xi / 2$, then, for any $a \in \operatorname{cl} B\left(a^{*}, \beta\right)$, by (62), for some $a^{+} \in A, \gamma \in[0,1]$,

$$
\left|\hat{v}-\left(q^{a^{+}, a}-\gamma b\right)\right|<\varsigma / 4
$$

We can even assume $a^{+} \in A_{a}$ by continuity of $\breve{a} \rightarrow q^{\breve{a}, a}$. By (61), for $\tilde{\varepsilon}=\varsigma(1 / 4)$, for some $a^{\prime} \in A$, and some arbitrarily small $\delta \in(0,1 / 2)$,

$$
\left|H\left(a^{\prime}\right)-H(a)-\delta q^{a^{+}, a}\right| \leq \tilde{\varepsilon} \delta=(1 / 4) \delta \varsigma, \quad \sigma\left(a^{\prime}, a\right) \leq \tilde{K}^{\prime} \delta .
$$

Now, by (63), $\left|\delta q^{a^{+}, a}-\delta(\hat{v}+\gamma b)\right| \leq \varsigma \delta / 4$. Then, by (64),

$$
\left|H\left(a^{\prime}\right)-H(a)-\delta(\hat{v}+\gamma b)\right| \leq(1 / 2) \delta \varsigma=(1 / 2) \delta|\hat{v}| .
$$


$\left(a \in \operatorname{cl} B\left(a^{*}, \beta\right), a^{\prime} \in \operatorname{cl} B\left(a, \tilde{K}^{\prime} \delta\right), \quad \gamma \in[0,1]\right)$. In Theorem D, replace $H(a)$ by $H(a)-\lambda b, a$ by $(a, \lambda), a^{*}$ by $\left(a^{*}, 0\right)$ and $A$ by $A \times[0,1]$, and let $\hat{\mu}=\beta \varsigma, \quad \mu^{\prime}=\varsigma, \quad \hat{z}^{*}=0, \mu=1 / 2, \quad K=\tilde{K}^{\prime}$,

$\rho\left(\left(a^{\prime \prime}, \lambda^{\prime \prime}\right),(a, \lambda)\right)=\max \left\{\varsigma \sigma\left(a^{\prime \prime}, a\right), \tilde{K}^{\prime} \varsigma\left|\lambda^{\prime \prime}-\lambda\right|\right\}$. Then the conditions in Theorem $\mathrm{D}$ are satisfied when, in (59), $\left(a, a^{\prime}, \delta\right)$ is replaced by $\left((a, \lambda),\left(a^{\prime}, \lambda^{\prime}\right), \delta\right), \quad a^{\prime}$ as just constructed, $\lambda^{\prime}=\delta \gamma+\lambda \in[0,1], \quad(\delta<1 / 2$, for $(a, \lambda) \in \mathrm{cl} B\left(\left(a^{*}, 0\right), \hat{\mu}\right), \quad \sigma\left(a, a^{*}\right) \leq \beta$, and $\lambda \leq 1 / 2$ as

$\left.\tilde{K}^{\prime} \varsigma|\lambda| \leq \hat{\mu}=\beta \varsigma \leq \tilde{K}^{\prime} \varsigma / 2\right)$. Thus, we get that, for all $\theta>0$ small enough, $H\left(a^{*}\right)+\theta b=H(a)-\tilde{\gamma} b$ for some $(a, \tilde{\gamma}) \in A \times[0,1]$, or $H\left(a^{*}\right)+(\tilde{\gamma}+\theta) b=H(a)$.

Remark H. If $q^{a^{*}, a^{*}}=0$, then $\tilde{\gamma}+\theta>0$ can be taken to be arbitrary small ( $b$ can be replaced by $\breve{\beta} b$ for any $\breve{\beta} \in(0,1])$. Hence, in this case, $H\left(a^{*}\right)+\hat{\gamma} b=H(a)$ holds for some arbitrarily small $\hat{\gamma}>0$.

Corollary I Let $\hat{Y}$ be a normed space, let $\gamma^{*}>0$, let $\tilde{A}$ be a complete pseudometric space with metric $\bar{\sigma}^{*}$ and let $\tilde{A}_{\tilde{a}}, \tilde{a} \in \tilde{A}$ be dense in $\tilde{A}$. Assume that $\tilde{a}^{*}$ is a given element in $\tilde{A}$, let $\tilde{H}(\tilde{a})=(\hat{H}(\tilde{a}), \breve{H}(\tilde{a})): \tilde{A} \rightarrow \hat{Y} \times \mathbb{R}: \tilde{Y}$ be continuous. Let $\tilde{q}^{\tilde{q}^{\prime}, \tilde{a}}=\left(\hat{q}^{\tilde{a}^{\prime}, \tilde{a}}, \breve{q}^{\tilde{a}^{\prime}, \tilde{a}}\right) \in \hat{Y} \times \mathbb{R}, \quad \tilde{a}^{\prime}, \tilde{a} \in \tilde{A}$. Assume that (61) and (62) are satisfies for $\left(H, q^{a^{\prime}, a}, A, A_{a}, Y, a^{*}, a, a^{\prime}, a^{+}, \tilde{\mu}, \sigma, \tilde{K}^{\prime}\right)$ replaced by $\left(\tilde{H}, \tilde{q}^{\tilde{a}^{\prime}, \tilde{a}}, \tilde{A}, \tilde{A} \tilde{a}, \tilde{Y}, \tilde{a}^{*}, \tilde{a}, \tilde{a}^{\prime}, \tilde{a}^{+}, \gamma^{*}, \bar{\sigma}^{*}, \tilde{K}_{*}^{\prime}\right)$ and also that $\tilde{a} \rightarrow \tilde{q}^{\tilde{q}^{\prime}, \tilde{a}}$ is continuous at $\tilde{a}^{*}$ for any $\tilde{a}^{\prime} \in \tilde{A}_{\tilde{a}^{*}}$ and that $\tilde{a}^{\prime} \rightarrow \tilde{q}^{\tilde{a}^{\prime}, \tilde{a}}$ is continuous for any $\tilde{a} \in \tilde{A}$, with $\tilde{q}^{\tilde{a}, \tilde{a}}=0$ for all $\tilde{a}$. Assume, for some given $\hat{y} \in \hat{Y}$, that

$\breve{H}\left(\tilde{a}^{*}\right)=\max _{\tilde{a} \in\{\tilde{\{} \in \tilde{A}: \hat{H}(\tilde{a}) \hat{\tilde{y}}\}} \breve{H}(\tilde{a})$. Assume also, for some $\varepsilon>0$, some $\breve{z}^{*} \in \hat{Y}$, that $B\left(\breve{z}^{*}, \varepsilon\right) \subset$ clco $\hat{q}^{\tilde{A}, \tilde{a}}$ for all $\tilde{a} \in\left\{\tilde{a} \in \tilde{A}, \bar{\sigma}^{*}\left(\tilde{a}, \tilde{a}^{*}\right) \leq \gamma^{*}\right\}$. Assume, finally, that $M:=\sup _{\tilde{a} \in B\left(\tilde{a}^{*}, \gamma^{*}\right)} \sup _{\tilde{a}^{\prime} \in \tilde{A}}\left|\widetilde{q}^{\tilde{a}^{\prime}, \tilde{a}}\right|<\infty$. Then, for some nonzero continuous linear functional $\left(\hat{y}^{*}, \breve{\lambda}\right)$ on $\hat{Y} \times \mathbb{R}, \quad \breve{\lambda}$ a number $\geq 0$, we have $\left\langle\hat{q}^{\tilde{a}^{\prime}, \tilde{a}^{*}}, \hat{y}^{*}\right\rangle+\breve{\lambda} \widetilde{q}^{\tilde{a}^{\prime}, \tilde{a}^{*}} \leq 0$ for all $\tilde{a}^{\prime} \in \tilde{A}$.

Proof. Define $z^{* *}=\left(\breve{z}^{*} / 2-2 M\right)$, and $\varepsilon^{* *}=\min \{\varepsilon / 2,2 M\}$. Define $K^{\tilde{a}}:=\operatorname{clco}\left\{\left(\hat{q}^{\tilde{q}^{\prime}, \tilde{a}}, \tilde{q}^{\tilde{q}^{\prime}, \tilde{a}}+\gamma\right): \tilde{a}^{\prime} \in \tilde{A}, \gamma \in[-8 M, 0]\right\}$. Evidently, for $\tilde{a} \in\left\{\tilde{a}: \bar{\sigma}^{*}\left(\tilde{a}, \tilde{a}^{*}\right) \leq \gamma^{*}\right\}$,

$B\left(\breve{z}^{*}, \varepsilon\right) \times\{0\} \subset \operatorname{clco}\left\{\left(\hat{q}^{\tilde{a}^{\prime}, \tilde{a}}, \breve{q}^{\tilde{a}^{\prime}, \tilde{a}}+\gamma\right): \tilde{a}^{\prime} \in \tilde{A}, \gamma=-\widetilde{q}^{\tilde{a}^{\prime}, \tilde{a}}\right\} \subset K^{\tilde{a}}$ and $\{0\} \times\{\beta:|\beta-4 M|<4 M\}=\{0\} \times(-8 M, 0) \subset \operatorname{clco}\left\{\left(\hat{q}^{\tilde{a}, \tilde{a}}, \tilde{q}^{\tilde{a}, \tilde{a}}+\gamma\right): \gamma \in(-8 M, 0)\right\}$, so $B\left(z^{* *}, \varepsilon^{* *}\right) \subset(1 / 2)\left[B\left(\breve{z}^{*}, \varepsilon\right) \times\{0\}\right]+(1 / 2)[\{0\} \times(-8 M, 0)] \subset K^{\tilde{a}}$. Assume by contradiction that $(0, \zeta)$ belongs to int $K^{\tilde{a}^{*}}$ for some $\zeta>0$. Define $A=\tilde{A} \times[-9 M, 0]$, and for $a=(\tilde{a}, \alpha) \in A, \quad a^{\prime}=\left(\tilde{a}^{\prime}, \alpha^{\prime}\right) \in A$, let $A_{a}=\tilde{A}_{\tilde{a}} \times[-9 M, 0], \quad q^{a^{\prime}, a}=\left(\hat{q}^{\tilde{a}^{\prime}, \tilde{a}}, \widetilde{q}^{\tilde{a}^{\prime}, \tilde{a}}+\alpha^{\prime}-\alpha\right)$ and $\sigma\left(a, a^{\prime}\right)=\max \left\{\bar{\sigma}^{*}\left(\tilde{a}^{\prime}, \tilde{a}\right),\left|\alpha^{\prime}-\alpha\right| \tilde{K}_{*}^{\prime} / 9 M\right\}$, and let $H(a)=(\hat{H}(\tilde{a}), \breve{H}(\tilde{a})+\alpha)$, $a=(\tilde{a}, \alpha)$. Then, for $\tilde{K}^{\prime}=\tilde{K}_{*}^{\prime}, \tilde{\mu}=\min \left\{\gamma^{*}, \tilde{K}^{\prime} / 9\right\} \quad$ (62) and (61) are evidently satisfied, (the latter for $\alpha^{\prime}=\delta \alpha^{+}+(1-\delta) \alpha$ when $a^{+}=\left(\tilde{a}^{+}, \alpha^{+}\right)$). Obviously, $B\left(z^{* *}, \varepsilon^{* *}\right) \subset \operatorname{clcoq}^{A, a}$ for each $a=(\tilde{a}, \alpha) \in \operatorname{cl} B\left(a^{*}, \tilde{\mu}\right)$ where $a^{*}=\left(\tilde{a}^{*}, 0\right) \quad \Leftrightarrow$ $\left.|\alpha| \leq M \Rightarrow[-8 M, 0] \subset[-9 M, 0]-\alpha \Rightarrow \operatorname{clcoq}^{A, a} \supset K^{\tilde{a}}\right)$. Hence, by the preceding corollary, for some $\eta>0, \eta(0, \zeta)+H\left(a^{*}\right)=H(a)$ for some $a=(\tilde{a}, \alpha) \in \tilde{A} \times[-9 M, 0]$. Hence, $\hat{H}(\tilde{a})=\hat{H}\left(\tilde{a}^{*}\right)=\hat{y}, \breve{H}(\tilde{a})+\alpha=\eta \zeta+\breve{H}\left(\tilde{a}^{*}\right)$, or $\breve{H}(\tilde{a})=\breve{H}\left(\tilde{a}^{*}\right)+\eta \zeta-\alpha>\breve{H}\left(\tilde{a}^{*}\right)$, contradicting optimality. Thus the set 
$L=\{(0, \zeta): \zeta>0\}$ is disjoint from int $K^{\tilde{a}^{*}}$, so the convex set $L$ can be separated from the convex set int $K^{\tilde{a}^{*}}$ by a nonzero continuous linear functional $y^{*}=\left(\hat{y}^{*}, \breve{\lambda}\right)$ such that $\left\langle K^{\tilde{a}^{*}}, y^{*}\right\rangle \leq 0 \leq\left\langle L, y^{*}\right\rangle, \quad\left(0 \in K^{\tilde{a}^{*}}\right)$, which implies $\breve{\lambda} \geq 0$.

Lemma J. Let $z(t, \omega), \breve{z}(t, \omega), \hat{z}(t, \omega) \in L_{2}\left(J \times \Omega, \mathbb{R}^{n_{*}}\right)$. Let

$\breve{f}(t, z): J \times \mathbb{R}^{n_{*}} \rightarrow \mathbb{R}^{n_{* *}}$ be continuous in $z$ and measurable in $t$. Assume that $\breve{f}_{z}(t, z)$ exists for all $(t, z)$ and that $\left|\breve{f}_{z}(t, z)\right| \leq \tilde{M}$ for all $(t, z)$. Let $z^{\prime} \rightarrow \breve{f}_{z}\left(t, z(t, \omega)+z^{\prime}\right)$ be continuous in $z^{\prime}$ at $z^{\prime}=0$, uniformly in $t, \omega$. Let $\operatorname{essup}_{t}|\breve{z}(t, .)|_{2} \leq 1,\left|\operatorname{essup}_{t} \hat{z}(t, .)\right|_{2}<\infty$. When $\delta \downarrow 0$, then

$$
\operatorname{essup}_{t}\left|\left\{\breve{f}_{z}(t, z(t, .)+\delta \breve{z}(t, .))-\breve{f}_{z}\left(t, x^{*}(t, .)\right)\right\}[\hat{z}(t, .)]\right|_{2}
$$

$\rightarrow 0$ uniformly in $\breve{Z}(.,$.$) such that \operatorname{essup}_{t}|\breve{z}(t, .)|_{2} \leq 1$.

Proof. Let an error function $e(d)$ be a nonnegative function on $[0, \infty)$ such that $e(d) \downarrow e(0)=0$ when $d \downarrow 0$. By continuity of $z^{\prime} \rightarrow \breve{f}_{z}\left(t, z(t, \omega)+z^{\prime}\right)$, uniformly in $t, \omega$, there exists an increasing error function $e(d)$ such that $\left|\breve{f}_{z}\left(t, z(t, \omega)+z^{\prime}\right)-\breve{f}_{z}(t, z(t, \omega))\right| \leq e\left(\left|z^{\prime}\right|\right)$ for all $z^{\prime}, t, \omega \quad$. Let $M(\omega)=\left\{t \in J: \hat{z}(t, \omega) \leq \operatorname{essup}_{t} \hat{z}(t, \omega)\right\} \quad$. Evidently, $\int_{J} 1_{M(\omega)}(t) \mathrm{d} t=1$, and for a.e. $t, 1_{M(\omega)}(t)=1$ a.s. $^{3}$

Suppose by contradiction that some $\varepsilon>0$ exists, such that, for each $k=1,2, \cdots$, there exist $\left(\delta_{k}, \breve{z}_{k}(.,).\right)$ such that, for

$$
\begin{gathered}
\varsigma_{k}(t, \omega):=\left|\left\{\breve{f}_{z}\left(t, z(t, \omega)+\delta_{k} \breve{z}_{k}(t, \omega)\right)-\breve{f}_{z}(t, z(t))\right\}[\hat{z}(t, \omega)]\right| \\
\operatorname{essup}_{t}\left|\varsigma_{k}(t, .)\right|_{2} \geq \varepsilon,
\end{gathered}
$$

and $\delta_{k}<1 / k, \operatorname{essup}_{t}|\breve{z}(t, .)|_{2} \leq 1$. Now for a.e. $t,\left|\varsigma_{k}(t, .)\right|_{2}=\left|1_{M(.)}(t) \varsigma_{k}(t, .)\right|_{2}$, so $\operatorname{essup}_{t}\left|1_{M(.)}(t) \varsigma_{k}(t, .)\right|_{2} \geq \varepsilon$. Let $\quad M^{\prime}=\left\{t:\left|\breve{z}_{k}(t, .)\right|_{2} \leq \operatorname{essup}_{t}\left|\breve{z}_{k}(t, .)\right|_{2}\right.$ for all $\left.k\right\}$. The set $M^{\prime}$ has full Lebesgue measure $\left(\operatorname{meas}\left(M^{\prime}\right)=\right.$ meas $\left.(J)\right)$. Then there must exist $t_{k} \in M^{\prime}$ such that

$$
\left|1_{M(.)}\left(t_{k}\right) \varsigma_{k}\left(t_{k}, .\right)\right|_{2} \geq \varepsilon / 2 .
$$

Now,

$$
\begin{aligned}
\zeta^{k}(\omega) & :=\left|1_{M(\omega)}\left(t_{k}\right) \varsigma_{k}\left(t_{k}, \omega\right)\right| \leq e\left(\delta_{k}\left|\breve{z}_{k}\left(t_{k}, \omega\right)\right|\right)\left|1_{M(\omega)}\left(t_{k}\right) \hat{z}\left(t_{k}, \omega\right)\right| \\
& \leq e\left(\delta_{k}\left|\breve{z}_{k}\left(t_{k}, \omega\right)\right|\right) \cdot \operatorname{essup}_{t}|\hat{z}(t, \omega)|=: \alpha_{k}(\omega) .
\end{aligned}
$$

There exists an increasing concave error function $e^{*}(d) \geq e(d)$, see Lemma $\mathrm{K}$ below. Evidently, by Jensen's inequality,

$$
\begin{aligned}
& E\left[e\left(\delta_{k}\left|\breve{z}_{k}\left(t_{k}, \omega\right)\right|\right)\right] \leq E\left[e^{*}\left(\delta_{k}\left|\breve{z}_{k}\left(t_{k}, \omega\right)\right|\right)\right] \leq e^{*}\left(\delta_{k} E\left[\left|\breve{z}\left(t_{k}, .\right)\right|\right]\right) . \\
& \leq e^{*}\left(\delta_{k}\left|\breve{z}\left(t_{k}, .\right)\right|_{2}\right) \leq e^{*}\left(\delta_{k} \operatorname{essup}_{t}|\breve{z}(t, .)|_{2}\right) \leq e^{*}\left(\delta_{k}\right)
\end{aligned} .
$$

So a subsequence $\beta_{k_{j}}(\omega)$ of $\beta_{k}:=e\left(\delta_{k}\left|\breve{z}_{k}\left(t_{k}, \omega\right)\right|\right)$ converges a.s. to zero, hence ${ }^{3}$ Note that $\int_{J} 1_{M(\omega)}(t) \mathrm{d} t=1$, for all $\omega$, so $1=E\left[\int_{J} 1_{M(\omega)}(t) \mathrm{d} t\right]=\int_{J} E\left[1_{M(\omega)}(t)\right] \mathrm{d} t$ which implies that $E\left[1_{M(\omega)}(t)\right]=1$ for a.e. $t$ and hence, for a.e. $t$, that $1_{M(\omega)}(t)=1$ a.s. 
also $\alpha_{k_{j}}(\omega)$ converges a.s. to zero ( $\operatorname{essup}_{t}|\hat{z}(t, \omega)|$ is a.s. finite by the assumption on $\hat{z}(.,$.$) in the Lemma). Moreover,$

$\left|\zeta^{k}(\omega)\right| \leq 2 \tilde{M}\left|1_{M(\omega)}\left(t_{k}\right) \hat{z}\left(t_{k}, \omega\right)\right| \leq 2 \tilde{M} \operatorname{essup}_{t}|\hat{z}(t, \omega)|, \operatorname{essup}_{t}|\hat{z}(t,)$.$| being a L_{2}$ -

function by the assumption on $\hat{z}(.,$.$) in the Lemma. By dominated$ convergence $\left|\zeta^{k_{j}}(.)\right|_{2} \rightarrow 0$ when $k_{j} \rightarrow \infty$, and a contradiction of (67) is obtained.

Lemma $\mathrm{K}$. Let $e(d), d \in[0,1]$ be an increasing error function. There exists an increasing concave error function $e^{*}(d)$ such that $e^{*}(.) \geq e($.$) .$

Proof. Let $C=\left\{\left(1 / 2^{n+1}, e\left(1 / 2^{n}\right)\right): n=0,1, \cdots\right\}$ and let $C^{\prime}=\{(0,0)\} \cup C$. The set $C^{\prime}$ is compact. There is a line through $\left(t_{1}, e_{1}\right)=(1 / 2, e(1))$ with minimal positive slope touching $C^{\prime}$ "to the left of" $\left(t_{1}, e_{1}\right)$ at some perhaps nonunique point $\left(t_{2}, e_{2}\right)=\left(1 / 2^{n_{2}+1}, e\left(1 / 2^{n_{2}}\right)\right), n_{2} \geq 1$. Here, "touching" means that the line contains a point of $C^{\prime \prime}:=C^{\prime} \cap\{[0,1 / 2) \times \mathbb{R}\}$, but no point in $C^{\prime \prime}$ lies strictly above the line. We choose $n_{2}>n_{1}=0$ as large as possible (perhaps $n_{2}=\infty$ ). All points $\left(1 / 2^{n+1}, e\left(1 / 2^{n}\right)\right), 0 \leq n \leq n_{2}$, ly below or on the segment $S_{1}$ between $\left(t_{2}, e_{2}\right)$ and $\left(t_{1}, e_{1}\right)$. If $t_{2}>0$, repeat the construction by replacing $\left(t_{1}, e_{1}\right)$ by $\left(t_{2}, e_{2}\right)$. The segment $S_{2}$ so obtained between $\left(t_{2}, e_{2}\right)$ and some $\left(t_{3}, e_{3}\right)=\left(1 / 2^{n_{3}+1}, e\left(1 / 2^{n_{3}}\right)\right), n_{3}>n_{2}, n_{3}$ chosen as large as possible, has a slope strictly larger than the slope of $S_{1}$, otherwise $n_{2}$ would not be as large as possible. All points $\left(1 / 2^{n+1}, e\left(1 / 2^{n}\right)\right), n_{2} \leq n \leq n_{3}$, ly below or on the segment $S_{2}$. Continue this prosess. The points $t_{i}>0$ obtained equals $1 / 2^{n_{i}}$ for some increasing subsequence $n_{i}$ of $0,1,2, \cdots$ Perhaps $t_{i^{\prime}}=0 \quad\left(n_{i^{\prime}}=\infty\right)$ for some $i^{\prime}$, or $t_{i}>0$ for $i=1,2, \cdots$ in which case $e_{i}=e\left(1 / 2^{n_{i}}\right) \rightarrow 0$. The union of the segments $S_{i}$ forms the graph of an increasing concave error function $e^{*}(d)$. We have that the points $\left(1 / 2^{n+1}, e^{*}\left(1 / 2^{n+1}\right)\right)$ on the segments have the property that they "dominate" the points $\left(1 / 2^{n+1}, e\left(1 / 2^{n}\right)\right)$ in the sense that $e\left(1 / 2^{n}\right) \leq e^{*}\left(1 / 2^{n+1}\right)$, and for $n=n_{i}$ we have $e\left(1 / 2^{n_{i}}\right)=e^{*}\left(1 / 2^{n_{i}+1}\right)$. Then for all $n \geq 1, n \geq n_{i}+1$, for $d \in\left(1 / 2^{n+1}, 1 / 2^{n}\right]$,

$e(d) \leq e\left(1 / 2^{n}\right) \leq e^{*}\left(1 / 2^{n+1}\right) \leq e^{*}(d) \leq e^{*}\left(1 / 2^{n}\right) \leq e^{*}\left(1 / 2^{n_{i}+1}\right)=e\left(1 / 2^{n_{i}}\right)$. (If

$t_{i^{\prime}}=0$, the segment $S_{i^{\prime}-1}$ contains $(0,0)$ as well as $\left(1 / 2^{n_{i^{\prime}-1}+1}, e\left(1 / 2^{n_{i^{\prime}-1}}\right)\right)$, so $e^{*}(d)$ is linear on $\left(0,1 / 2^{n_{i-1}+1}\right]$ and $e(d) \leq e^{*}(d)$ for $d \in\left(0,1 / 2^{n_{i^{\prime}-1}+1}\right]$, $e^{*}(0)=0$.)

Remark L (Proof of (29)) Let

$z(.,.) \in L_{2}^{* * n}=\left\{z(.,.) \in L_{2}^{\text {prog }}\left(J \times \Omega, \mathbb{R}^{n}\right):\|z(., .)\|_{2}^{*}<\infty\right\}$ and let $\|z(., .)\|_{2}^{*}=\alpha<\infty$. Let $\operatorname{essup}_{t}|z(t, \omega)|=\beta(\omega)$ and let $M_{N}=\{\omega: \beta(\omega) \leq N\}$. Then

$\beta(\omega)-\beta(\omega) 1_{M_{N}}(\omega) \rightarrow 0$ a.s. when $N \rightarrow \infty$ as $\beta($.) is a.s. finite, because $E \beta^{2} \leq \alpha^{2}$. Then

$\operatorname{essup}_{t}\left|z(t, \omega)-z(t, \omega) 1_{M_{N}}(\omega)\right|=\operatorname{essup}_{t}|z(t, \omega)| 1_{C_{M_{N}}}=\beta(\omega) 1_{C_{M_{N}}} \rightarrow 0 \quad$ a.s. and by Lebesgue's dominated convergence theorem also in $L_{2}$. So $L_{\infty}^{\text {prog }}\left(J \times \Omega, \mathbb{R}^{n}\right)$ is $\|\cdot\|_{2}^{*}$-dense in $L_{2}^{* * n}$ (and then also $\|\cdot\|_{2}$-dense). Evidently, $L_{\infty}^{\text {prog }}\left(J \times \Omega, \mathbb{R}^{n}\right) \subset L_{2}^{* *, n}$, moreover, as shown, $L_{2}^{* *, n} \subset \mathrm{cl}_{2} L_{\infty}^{\text {prog }}\left(J \times \Omega, \mathbb{R}^{n}\right)$, so $L_{2}^{* n}=\mathrm{Cl}_{2} L_{2}^{* * n}=\mathrm{Cl}_{2} L_{\infty}^{\text {prog }}\left(J \times \Omega, \mathbb{R}^{n}\right)$. 
If $u \in L_{2}^{\text {prog }}\left(J \times \Omega, \mathbb{R}^{n * *}\right)$, then $\left\|x^{u}(. . .)\right\|_{2}^{*}<0$ by (57) in Appendix. If $u^{\prime}(.,.) \in L_{2}^{\text {prog }}\left(J \times \Omega, \mathbb{R}^{n * *}\right), \quad u^{\prime \prime} \in U^{* *}$, then

$$
\begin{aligned}
& \left|f\left(., x^{u^{\prime}}(., .), u^{\prime \prime}(., .)\right)-f\left(., x^{u^{*}}(., .), u^{*}(., .)\right)\right| \\
& \leq\left|f\left(., x^{u^{\prime}}(., .), u^{\prime \prime}(., .)\right)-f\left(., x^{u^{*}}(., .), u^{\prime \prime}(., .)\right)\right| \\
& \quad+\left|f\left(., x^{u^{*}}(., .), u^{\prime \prime}(., .)\right)-f\left(., x^{u^{*}}(., .), u^{*}(., .)\right)\right| \\
& \leq M^{+}\left|x^{u^{\prime}}(., .)-x^{*}(., .)\right|+M^{+}\left|u^{\prime \prime}(., .)-u^{*}(. .)\right|,
\end{aligned}
$$

so $f\left(., x^{u^{\prime}}(.,),. u^{\prime \prime}(.,).\right)-f\left(., x^{u^{*}}(.,),. u^{*}(.,).\right) \in L_{2}^{*, n^{*}}$. Because

$\left|f\left(., x^{u^{\prime}}(.,),. u^{\prime \prime}(.,).\right)-f\left(., x^{u^{\prime}}(.,),. u^{\prime}(.,).\right)\right| \leq M^{+}\left|u^{\prime \prime}(.,)-.u^{\prime}(.,).\right|$, then

$f\left(., x^{u^{\prime}}(.,),. u^{\prime \prime}(.,).\right)-f\left(., x^{u^{*}}(.,),. u^{*}(.,).\right) \rightarrow$

$f\left(., x^{u^{\prime}}(.,),. u^{\prime}(.,).\right)-f\left(., x^{u^{*}}(.),. u^{*}(.).\right)$ in $\|\cdot\|_{2}$ when (in $\left.\|\cdot\|_{2}\right)$

$u^{\prime \prime} \rightarrow u^{\prime} \in \mathrm{cl}_{2} L_{\infty}^{\text {prog }}\left(J \times \Omega, \mathbb{R}^{n^{* *}}\right), u^{\prime \prime} \in U^{* *}$, hence

$f\left(., x^{u^{\prime}}(.,),. u^{\prime}(.,).\right)-f\left(., x^{u^{*}}(.,),. u^{*}(.,).\right) \in L_{2}^{*, n}$ when $u^{\prime} \in \mathrm{cl}_{2} U^{* *}$.

Now, $f_{u}\left(., x^{u_{*}}(.,),. u_{*}(.,).\right)[u(.,).] \in L_{\infty}^{\text {prog }}\left(J \times \Omega, \mathbb{R}^{n^{*}}\right)$ when $u \in L_{\infty}^{\text {prog }}\left(J \times \Omega, \mathbb{R}^{n^{* *}}\right)$, by $\left|f_{u}\right| \leq M^{+}$, and then

$f_{u}\left(., x^{u_{*}}(.,),. u_{*}(.,).\right)\left[u_{n}(.,).\right] \rightarrow f_{u}\left(., x^{u_{*}}(.,),. u_{*}(.,).\right)[u(.,)$.$] \quad in \|.\|_{2_{* *}}$ when $u_{n} \rightarrow u \quad$ in $\|\cdot\|_{2}, \quad u \in \operatorname{cl}_{2} L_{\infty}^{\text {prog }}\left(J \times \Omega, \mathbb{R}^{n^{* *}}\right), \quad u_{n} \in L_{\infty}^{\text {prog }}\left(J \times \Omega, \mathbb{R}^{n^{* *}}\right)$, so $f_{u}\left(., x^{u_{*}}(.,),. u_{*}(.,).\right)[u(.,).] \in \mathrm{cl}_{2} L_{\infty}^{\text {prog }}\left(J \times \Omega, \mathbb{R}^{n^{*}}\right)$ when

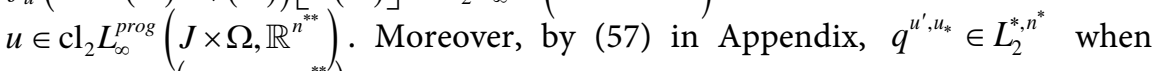
$u^{\prime}, u_{*} \in L_{2}^{\text {prog }}\left(J \times \Omega, \mathbb{R}^{n^{* *}}\right)$, so

$f_{x}\left(., x^{u_{*}}(.,),. u_{*}(.,).\right) q^{u^{\prime}, u_{*}}(.,.) \in L_{2}^{*, n^{*}} \subset \mathrm{cl}_{2} L_{\infty}^{\text {prog }}\left(J \times \Omega, \mathbb{R}^{n^{*}}\right)$ when

$u^{\prime}, u_{*} \in L_{2}^{\text {prog }}\left(J \times \Omega, \mathbb{R}^{n^{* *}}\right)$.

Hence $\pi \dot{x}^{u}(.,)-.\pi \dot{x}^{*}($.$) as well as \pi \dot{q}^{u, u_{*}}(.,$.$) belong to$

$\mathrm{cl}_{2} L_{\infty}^{\text {prog }}\left(J \times \Omega, \mathbb{R}^{m^{*}}\right)=L_{2}^{*, m^{*}}$ when $u, u_{*} \in \mathrm{cl}_{2} U^{* *}$

$\left(\Rightarrow u-u_{*}=u-u^{*}-\left(u_{*}-u^{*}\right) \in \mathrm{cl}_{2} L_{\infty}^{\operatorname{prog}}\left(J \times \Omega, \mathbb{R}^{n^{* *}}\right)\right)$.

Remark M (Completeness of $\mathrm{Cl}_{2} U^{* *}$ )

Evidenly, $L_{2}^{\text {prog }}\left(J \times \Omega, \mathbb{R}^{n^{* *}}\right)$ is complete, so a $|\cdot|_{2}$-Cauchy-sequence $z_{n}(.,$. in $\mathrm{cl}_{2} U^{* *}$ is $|\cdot|_{2}$-convergent to some $z(.,.) \in L_{2}^{\text {prog }}\left(J \times \Omega, \mathbb{R}^{n^{* *}}\right)$. Then for a subsequence $z_{n_{j}}(.$.$) , for a.e. t,\left|z_{n_{j}}(t, .)-z(t, .)\right|_{2} \rightarrow 0$. From this we get that, for any $\varepsilon>0$, if $\operatorname{essup}_{t} E\left|z_{n}(t, .)-z_{m}(t, .)\right|^{2} \leq \varepsilon$ when $n, m$ are large, then for a.e. $t$ even $E\left|z_{n}(t, .)-z(t, .)\right|^{2} \leq \varepsilon$, so $\operatorname{essup}_{t} E\left|z_{n}(t, .)-z(t, .)\right|^{2} \leq \varepsilon$, which means that $\mathrm{cl}_{2} U^{* *}$ is $\|\cdot\|_{2}$-complete.

Remark $\mathbf{N}\left(E\left[g\left[\Phi_{t}\right]\right] \rightarrow E\left[g \mid \Phi_{s}\right]\right.$ in $L_{2}$-norm and a.s. when $\left.t \downarrow s\right)$.

Let $g \in L_{2}\left(\Omega, \Phi, \mathbb{R}^{n}\right)$, and let $t_{j} \downarrow s$. By weak compactness, a convex combination $g_{j}:=\sum_{i} \lambda_{i_{j}} E|g| \Phi_{t_{n_{j}}}, \quad t_{n_{j}^{i}}>s, n_{j}^{i} \geq j$, converges in $|\cdot|_{2}$ and also almost uniformly to some $\Phi_{s}^{n_{j}^{i}}$-measurable limit ${ }^{4} g^{*}$, the latter convergence by considering subsequences if necessary. By $|\cdot|_{2}$-convergence, $g^{*}$ evidently satisfies

$$
\begin{aligned}
g^{*} & =E\left[g^{*} \mid \Phi_{s}\right]=\lim _{j} E\left[\sum_{i} \lambda_{i_{j}} E\left[g \mid \Phi_{t_{n_{j}}}\right] \mid \Phi_{s}\right] . \\
& =\lim _{j}\left[\sum_{i} \lambda_{i_{j}} E\left[g \mid \Phi_{s}\right]=E\left[g \mid \Phi_{s}\right]\right.
\end{aligned}
$$

${ }^{4}$ A.s. convergence of the component $g_{j}^{k}$ to $g^{* k}, k=1, \cdots, n$, means $\left\{\omega: g^{* k} \geq a\right\} \in \Phi_{t j^{\prime}}$ for any given $j^{\prime}$ (a arbitrary), hence $\left\{\omega: g^{* k} \geq a\right\} \in \bigcap_{j^{\prime}} \Phi_{t_{j^{\prime}}}=\Phi_{s}$. 
Let $\varepsilon>0$ and $\varepsilon^{\prime}>0$ be arbitrary, let $M$ be a set on which $g_{j}$ converges uniformly, $P(C M) \leq \varepsilon^{\prime}$, and let $j$ be the smallest $j$ such that $\left|g_{j}-g^{*}\right|_{2} \leq \varepsilon$ and $\left|1_{M}\left\{g_{j}-g^{*}\right\}\right|_{\infty} \leq \varepsilon$. Let $t^{j}=\min _{i} t_{n_{j}^{i}}>s$. Then, for $t \leq t^{j}, \quad s<t, \infty>q \geq 2$, using Jensens inequality twice,

$$
\begin{aligned}
& \left|1_{M}\left\{E\left[g \mid \Phi_{t}\right]-g^{*}\right\}\right|_{q} \\
& =\left|1_{M}\left\{E\left[\sum_{i} \lambda_{i_{j}} E\left[g \mid \Phi_{t_{n_{j}}}\right] \mid \Phi_{t}\right]-E\left[g^{*} \mid \Phi_{t}\right]\right\}\right|_{q} \\
& =\left|1_{M}\left\{E\left[g_{j} \mid \Phi_{t}\right]-E\left[g^{*} \mid \Phi_{t}\right]\right\}\right|_{q} \leq\left(E\left[1_{M} E\left\{\left|g_{j}-g^{*}\right| \mid \Phi_{t}\right\}^{q}\right]\right)^{1 / q} \\
& \leq\left(E\left[1_{M} E\left\{\left|g_{j}-g^{*}\right|^{q} \mid \Phi_{t}\right\}\right]\right)^{1 / q} \leq\left(\int_{M}\left|g_{j}-g^{*}\right|^{q} \mathrm{~d} P\right)^{1 / q} \leq \varepsilon .
\end{aligned}
$$

For $q=2$, this holds even for $M=\Omega$, yielding $|\cdot|_{2}$-convergence of $E\left[g \mid \Phi_{t}\right]$ when $t \downarrow s$, using $|\cdot|_{\infty}=\sup _{q}|\cdot|_{q}$ yields almost uniform convergence.

Remark O. In this remark let "tr" mean tranpose. Note that $E\left\langle z(),. p^{-}(b)\right\rangle=\left\langle C(T, b) z(),. v_{*}\right\rangle=\left\langle z(),. C(T, b)^{*} v_{*}\right\rangle \quad\left(z(.) \in L_{2}\left(\Omega, \Phi_{b}, \mathbb{R}^{n^{*}}\right)\right)$ and that, for $y(.) \in L_{2}\left(\Omega, \Phi_{t}, \mathbb{R}^{n^{*}}\right), t<b$,

$$
\begin{aligned}
& E\left\langle y(.), p^{-}(t)\right\rangle=\left\langle C(T, t) y(.), v_{*}\right\rangle=\left\langle C(T, b) C(b, y) y(.), v_{*}\right\rangle \\
& =\left\langle C(b, t) y(.), C(T, b)^{*} v_{*}\right\rangle=E\left\langle C(b, t) y(.), p^{-}(b)\right\rangle \\
& =E\left\langle y(.), C(b, t) p^{-}(b)\right\rangle=E\left\langle y(.), E\left[C(b, t)^{*} p^{-}(b) \mid \Phi_{\tau}\right]\right\rangle .
\end{aligned}
$$

Taking now $p^{-}(t)$ to be a row vector, then $p^{-}(t)$ satisfies $p^{-}(t)^{t r}=E\left[C(b, t)^{t r} p^{-}(b)^{t r} \mid \Phi_{t}\right]$. Letting $Y(t), \Psi(t), T, A(t), B_{j}(t)$ be the entities on pp. 348-350 in [6], for $A(t)=-\left(f_{x}^{*}(t)\right)^{t r}, B_{j}(t)=-\left(\bar{\sigma}_{x}^{j *}(t)\right)^{t r}$, $\theta=p^{-}(b)^{t r}, T=b$, we have that $\Psi(t)^{t r}=C(t, 0)$, and then $Y(t)=C(0, t)^{t r} E\left[C(b, 0)^{t r} \theta \mid \Phi_{t}\right]=E\left[C(b, t)^{t r} \theta \mid \Phi_{\tau}\right]$ by (2.20) in that book, so by (2.9) in that book, for some $q(t), p(t)=Y(t)^{t r}$ satisfies (54),(55).

Remark P (Proof of continuity of $y(.) \rightarrow\langle\pi C(1, s,) y.(), v$.$\rangle on$ $\left.L_{2}\left(\Omega, \Phi_{s}, \mathbb{R}^{n^{*}}\right), \quad s<T\right)$.

Let $T=1, \quad x_{0}=0, s<1$ and let $k$ be the smallest $k$ such that $s \leq 1-1 / 2^{k}$. Note that if $y(.) \in L_{2}\left(\Omega, \Phi_{s}, \mathbb{R}^{n^{*}}\right)$, then $\Pi_{k^{\prime}} y()=$.0 , for $k^{\prime}>k$, and that $\Pi_{i}|y(.)|_{2} \leq 2^{-i} \cdot{ }_{2}|y()$.$| . Hence$

$|y(.)|_{2}=\left|\sum_{i=1}^{k} \Pi_{i} y(.)\right|_{2} \leq \sum_{i=1}^{k} 2^{-i}\left({ }_{2}|y()|.\right) \leq{ }_{2}|y()|$. On the other hand ${ }_{2}|y()|=.\sup _{i} 2^{i}\left|\Pi_{i} y(.)\right|_{2} \leq 2^{k} 2 \cdot|y(.)|_{2}$. Hence, on $L_{2}\left(\Omega, \Phi_{s}, \mathbb{R}^{n^{*}}\right)$, the norms $|\cdot|_{2}$ and ${ }_{2}|\cdot|$ are equivalent. (Thus, the spaces $L_{2}\left(\Omega, \Phi_{s}, \mathbb{R}^{m^{*}}\right), \quad s<1$ are subspaces of $L^{2}$, in fact of $\operatorname{cl}_{(2)} L_{\infty}^{*}$, because $L_{2}\left(\Omega, \Phi_{s}, \mathbb{R}^{m^{*}}\right)=|\cdot|_{2}$-closure of $L_{\infty}\left(\Omega, \Phi_{s}, \mathbb{R}^{m^{*}}\right)$.) For $y=y(.) \in L_{2}\left(\Omega, \Phi_{s}, \mathbb{R}^{n^{*}}\right)$, define

$$
\begin{aligned}
q^{y}(t, s, \omega)= & y(\omega)+\int_{s}^{t} f_{x}\left(\breve{s}, x^{*}(\breve{s}, \omega), u^{*}(\breve{s}, \omega)\right) q^{y}(\breve{s}, s, \omega) \mathrm{d} \breve{s} \\
& +\int_{s}^{t} \bar{\sigma}_{x}\left(\breve{s}, x^{*}(\breve{s}, \omega), u^{*}(\breve{s}, \omega)\right) q^{y}(\breve{s}, s, \omega) \mathrm{d} B_{\breve{s}} .
\end{aligned}
$$


Then an application of Appendix, Lemma A gives that $\sup _{t, s, t \geq s}\left|q^{y}(t, s, .)\right|_{2} \leq D|y(.)|_{2}$ for some constant $D$ independent of $y($.$) . Then$ $\left\|1_{[s, 1]}(.) \partial \pi q^{y}(., s, .) / \partial t\right\|_{2} \leq D M^{+}|y(.)|_{2}$.

Because $|\Theta| \leq 4$ for $\|\cdot\|_{2} \rightarrow_{2}|\cdot|$, then

$$
\begin{aligned}
& { }_{2}\left|\pi q^{y}(1, s, .)-\pi y(.)\right| \\
& ={ }_{2}\left|\int_{0}^{1}\left[1_{[s, 1]}(t) \partial \pi q^{y}(t, s, .) / \partial t\right] \mathrm{d} t\right| \\
& \leq 4 \cdot\left\|1_{[s, 1]}(t) \partial \pi q^{y}(., s, .) / \partial t\right\|_{2} \leq 4 D M^{+}|y(.)|_{2} .
\end{aligned}
$$

Now, $\quad \pi y()+.\int_{0}^{1}\left[1_{[s, 1]}(t) \partial \pi q^{y}(t, s,.) / \partial t\right] \mathrm{d} t=\pi q^{y}(1, s,)=.\pi C(1, s,) y.($.$) \quad , so$ ${ }_{2}\left|\pi q^{y}(1, s,).\right| \leq 4 D^{+}|y(.)|_{2}+2^{k+1}|y(.)|_{2}$. For some constant $\gamma$, $|\langle z(), v\rangle.| \leq \gamma \cdot{ }_{2}|z()$.$| for z(.) \in \mathrm{cl}_{(2)} L_{\infty}^{*}$, so $|\langle\pi C(1, s,) y.(), v\rangle.| \leq 4 \gamma D M^{+}|y(.)|_{2}+\gamma 2^{k+1}|y(.)|_{2}, \quad y(.) \in L_{2}\left(\Omega, \Phi_{s}, \mathbb{R}^{n^{*}}\right)$.

Remark Q (Proof of $|\cdot|_{2}$-continuity of $t \rightarrow p^{-}(t), t<T$ ).

Let $T=1$, and fix $s^{\prime \prime} \in(0,1)$. By Remark $\mathrm{P}, y(.) \rightarrow\left\langle\pi C\left(1, s^{\prime \prime},.\right) y(), v.\right\rangle$ is $|\cdot|_{2}$-continuous in $L_{2}\left(\Omega, \Phi_{s^{\prime \prime}}, \mathbb{R}^{n^{*}}\right)$, so there exists an $L_{2}\left(\Omega, \Phi_{s^{\prime \prime}}, \mathbb{R}^{n^{*}}\right)$ function $p_{-}^{*}\left(s^{\prime \prime},.\right)$, such that $\left\langle\pi C\left(1, s^{\prime \prime},.\right) y(), v.\right\rangle=E\left\langle y(),. p_{-}^{*}\left(s^{\prime \prime},.\right)\right\rangle$. Define $p_{-}^{*}(s,)=.C\left(s^{\prime \prime}, s, .\right)^{*} p_{-}^{*}\left(s^{\prime \prime},.\right), s \leq s^{\prime \prime}$. Note that $C\left(s^{\prime \prime}, 0, \omega\right)=C\left(s^{\prime \prime}, s, \omega\right) C(s, 0, \omega)$, so $s \rightarrow C\left(s^{\prime \prime}, s, \omega\right)$ is continuous because $C\left(s^{\prime \prime}, s, \omega\right)=C\left(s^{\prime \prime}, 0, \omega\right) C(s, 0, \omega)^{-1}$ and $s \rightarrow C(s, 0, \omega)$ is continuous

(compare Theorem 6.14 p. 47 in [6], a similar theorem holds for random coeffficients). Because $\left|\sup _{s \leq s^{\prime \prime}} C\left(s^{\prime \prime}, s, .\right)\right|_{2}<\infty$ by Lemma A in Appendix, $s \rightarrow C\left(s^{\prime \prime}, s,.\right)$ is $|\cdot|_{2}$-continuous. Hence $s \rightarrow p_{-}^{*}(s,$.$) is a.s. and |\cdot|_{2}$ -continuous.

Evidently, for any $y(.) \in L_{2}\left(\Omega, \Phi_{s}, \mathbb{R}^{n^{*}}\right)$,

$$
\begin{aligned}
& \langle\pi C(1, s, .) y(.), v\rangle \\
& =\left\langle\pi C\left(1, s^{\prime \prime}, .\right) C\left(s^{\prime \prime}, s, .\right) y(.), v\right\rangle=E\left\langle C\left(s^{\prime \prime}, s, .\right) y(.), p_{-}^{*}\left(s^{\prime \prime}, .\right)\right\rangle \\
& =E\left\langle y(.), C\left(s^{\prime \prime}, s, .\right)^{*} p_{-}^{*}\left(s^{\prime \prime}, .\right)\right\rangle=E\left\langle y(.), p_{-}^{*}(s, .)\right\rangle .
\end{aligned}
$$

Then, for any $y(.) \in L_{2}\left(\Omega, \Phi_{s}, R^{n^{*}}\right)$,

$$
\begin{aligned}
& E\left\langle y(.), p_{-}(s, .)\right\rangle=\langle\pi C(1, s, .) y(.), v\rangle=E\left\langle y(.), p_{-}^{*}(s, .)\right\rangle \\
& =E E\left[\left\langle y(.), p_{-}^{*}(s, .)\right\rangle \mid \Phi_{s}\right]=E\left\langle y(.), E\left[p_{-}^{*}(s, .) \mid \Phi_{s}\right]\right\rangle .
\end{aligned}
$$

From this it follows that $p_{-}(s,)=.E\left[p_{-}^{*}(s,) \mid. \Phi_{s}\right]$ for all $s \in\left[0, s^{\prime \prime}\right]$.

Let $s, s<s^{\prime \prime}$ be given. Note that if $\phi(.) \in L_{2}\left(\Omega, \Phi_{s^{\prime \prime}}, \mathbb{R}^{n^{*}}\right)$ and $t \rightarrow \Phi_{t}$ is (also) left continuous, then $\lim _{s^{\prime} \uparrow s} E\left[\phi() \mid. \Phi_{s^{\prime}}\right]=E\left[\phi() \mid. \Phi_{s}\right]$ in $|\cdot|_{2}$ and a.s. , ${ }^{5}$ If $s^{\prime}=s_{k} \uparrow s$, this corollary ensures only $|\cdot|_{1}$-convergence of $E\left[\phi \mid \Phi_{s_{k}}\right]$ to $E\left[\phi \mid \Phi_{s}\right]$, but also of $E\left[|\phi|^{2} \mid \Phi_{s_{k}}\right]$ in $|\cdot|_{1}$ (to $E\left[|\phi|^{2} \mid \Phi_{s}\right]$, which means that $M \rightarrow E\left[1_{M} E\left[|\phi|^{2} \mid \Phi_{s_{k}}\right]\right]$ is equicontionuous, $M \in \Phi_{1}$ (the countable additivity is uniform). Then $E\left[\phi \mid \Phi_{s_{k}}\right] \rightarrow E\left[\phi \mid \Phi_{s}\right]$ in $|\cdot|_{2}$, because $1_{M}\left(E\left[\phi \mid \Phi_{s_{k}}\right]\right)^{2} \leq 1_{M} E\left[|\phi|^{2} \mid \Phi_{s_{k}}\right]$, so $M \rightarrow E\left\{1_{M}\left(E\left[\phi \mid \Phi_{s_{k}}\right]\right)^{2}\right\}$ is equicontionuous. 
see Corollary A.9, Appendix C in $[1]^{5}$ and $\lim _{s^{\prime} \downarrow s} E\left[\phi() \mid. \Phi_{s^{\prime}}\right]=E\left[\phi() \mid. \Phi_{s}\right]$ in $|.|_{2}$ and a.s., see Remark $\mathrm{N}$ in Appendix, or, for both these results, Theorem 6.23 in [10]. Using this for $\phi()=.p_{-}^{*}(s,$.$) and p_{-}\left(\hat{s}_{.}.\right)=E\left[p_{-}^{*}\left(\hat{s}_{,},.\right) \mid \Phi_{\hat{s}}\right]$ and continuity of $p_{-}^{*}(s,$.$) yield that s \rightarrow p_{-}(s,$.$) is a.s. and |\cdot|_{2}$-continuous, as we shall see: Fix any $s<s^{\prime \prime}$. When $s^{\prime}$ is close to $s$,

$p_{-}(s,)=.E\left[p_{-}^{*}(s,) \mid. \Phi_{s}\right] \approx E\left[p_{-}^{*}(s,) \mid. \Phi_{s^{\prime}}\right] \approx E\left[p_{-}^{*}\left(s^{\prime},.\right) \mid \Phi_{s^{\prime}}\right]=p_{-}\left(s^{\prime},.\right)$. This is obvious for the $|\cdot|_{2}$-norm, and also for a.s.-convergence, once we have shown that $E\left[p_{-}^{*}\left(s^{\prime},.\right) \mid \Phi_{s^{\prime}}\right] \approx E\left[p_{-}^{*}(s,) \mid. \Phi_{s^{\prime}}\right]$ almost uniformly. Note that by Egoroff's theorem, $p_{-}^{*}\left(s^{\prime},.\right) \rightarrow p_{-}^{*}(s,$.$) almost uniformly. Let$ $\kappa=\sup _{\hat{s} \leq s^{\prime \prime}}\left|p_{-}^{*}(\hat{s}, .)\right|_{2}<\infty$. For any $\varepsilon \in(0,1)$, for some set $M \in \Phi_{s^{\prime \prime}}$, with $E\left(1_{C M}\right) \leq \varepsilon^{2}$ and some $\delta>0,\left|1_{M}\left(p_{-}^{*}\left(s^{\prime}, .\right)-p_{-}^{*}(s, .)\right)\right|^{\infty} \leq \varepsilon$ when $\left|s^{\prime}-s\right| \leq \delta$. By almost uniform convergence again, we can find a set $M^{*} \in \Phi_{s^{\prime \prime}}$, with $E 1_{\mathrm{CM}^{*}}<\varepsilon$ and a $\delta^{*} \in(0, \delta]$ such that $\mid\left[1_{M^{*}}{ }^{{ }^{*}} E\left[1_{C M} \mid \Phi_{s^{\prime}}\right]-1_{M^{*}} E\left[1_{C M} \mid \Phi_{s}\right]^{\infty} \leq \varepsilon / 2 \quad\right.$ when $\quad\left|s^{\prime}-s\right| \leq \delta^{*}$. Defining $M^{\prime}=\left\{\omega: E\left[1_{C M} \mid \Phi_{s}\right]>\varepsilon\right\}$ and $M^{\prime \prime}=M^{*} \cap C M^{\prime}$, we have

$1_{M^{\prime \prime}} E\left[1_{C M} \mid \Phi_{s^{\prime}}\right] \leq 1_{M^{\prime \prime}}(\varepsilon+\varepsilon / 2)=1_{M^{\prime \prime}} 3 \varepsilon / 2$ and $1_{M^{\prime} \cap M^{*}} E\left[1_{C M} \mid \Phi_{s^{\prime}}\right] \geq 1_{M^{\prime} \cap M^{*}} \varepsilon / 2$ and hence $1_{M^{\prime} \cap M^{*}} \leq 1_{M^{\prime} \cap M^{*}} E\left[1_{C M} \mid \Phi_{s^{\prime}}\right](2 / \varepsilon)$ when $\left|s^{\prime}-s\right| \leq \delta^{*}$. Thus,

$$
\begin{aligned}
E 1_{M^{\prime} \cap M^{*}} & \leq E\left\{1_{M^{\prime} \cap M^{*}} E\left[1_{C_{M}} \mid \Phi_{s^{\prime}}\right] 2 / \varepsilon\right\} \\
& \leq E\left\{E\left[1_{C M} \mid \Phi_{s^{\prime}}\right] 2 / \varepsilon\right\} \leq(2 / \varepsilon) E\left\{1_{C M}\right\} \leq 2 \varepsilon
\end{aligned}
$$

when $\left|s^{\prime}-s\right| \leq \delta^{*}$. Then,

$$
\begin{aligned}
& 1_{M^{\prime \prime}} \mid E\left[p_{-}^{*}(s, .)-p_{-}^{*}\left(s^{\prime}, .\right) \mid \Phi_{s^{\prime}}\right] \\
& \leq 1_{M^{\prime \prime}}\left|E\left[1_{M}\left(p_{-}^{*}(s, .)-p_{-}^{*}\left(s^{\prime}, .\right)\right) \mid \Phi_{s^{\prime}}\right]\right| \\
& \quad+1_{M^{\prime \prime}}\left|E\left[1_{C M}\left(p_{-}^{*}(s, .)-p_{-}^{*}\left(s^{\prime}, .\right)\right) \mid \Phi_{s^{\prime}}\right]\right| \\
& \leq 1_{M^{\prime \prime}} \varepsilon+2 \kappa 1_{M^{\prime \prime}}\left(E\left[1_{C M} \mid \Phi_{s^{\prime}}\right]\right)^{1 / 2} \\
& \leq 1_{M^{\prime \prime}} \varepsilon+2 \kappa 1_{M^{\prime \prime}}(3 \varepsilon / 2)^{1 / 2} .
\end{aligned}
$$

So we have $\left|E\left[p_{-}^{*}(s,) \mid. \Phi_{s^{\prime}}\right]-E\left[p_{-}^{*}\left(s^{\prime},.\right) \mid \Phi_{s^{\prime}}\right]\right| \leq \varepsilon+2 \kappa(3 \varepsilon / 2)^{1 / 2}$ when $\left|s^{\prime}-s\right| \leq \delta^{*}$ and $s \in M^{\prime \prime}=M^{*} \cap C M^{\prime}$. Now,

$\complement M^{\prime \prime}=M^{\prime} \cup C M^{*}=\left(M^{\prime} \cap M^{*}\right) \cup C M^{*}$, and

$E 1_{\left(M^{\prime} \cap M^{*}\right) \cup C M^{*}}=E 1_{\left(M^{\prime} \cap M^{*}\right)}+E 1_{C M^{*}} \leq 2 \varepsilon+\varepsilon$. Hence,

$E\left[p_{-}^{*}(s,) \mid. \Phi_{s^{\prime}}\right] \rightarrow E\left[p_{-}^{*}\left(s^{\prime},.\right) \mid \Phi_{s^{\prime}}\right]$ almost uniformly.

Remark R (Proof of $(21) \Rightarrow(15)$, with $\breve{z}(.,)=$.0 , when $\left\|u^{*}\right\|_{2}^{*}<\infty$ )

Let $z(t, \omega)=\pi f_{u}\left(t, x^{u_{*}}(t, \omega), u_{*}(t, \omega)\right) u^{*}(t, \omega) \in L_{2}^{* m^{*}}$. For any $y(.,.) \in B_{1}^{*, m^{*}}$, there exists a $u^{\prime}(t, \omega) \in U$, with $\left|u^{\prime}(t, \omega)\right| \leq \beta_{0}|y(t, \omega)+z(t, \omega)|, \quad u^{\prime}(.,.) \in L_{2}^{* n^{* *}}$ (and with $\left\|u^{\prime}(., .)\right\|_{2} \leq \beta_{0}\left(1+M^{+}\left\|u^{*}\right\|_{2}\right)$ ), such that

$$
\begin{aligned}
& y(t, \omega)+z(t, \omega)=\pi f_{u}\left(t, x^{u_{*}}(t, \omega), u_{*}(t, \omega)\right) u^{\prime}(t, \omega) \text { and hence } \\
& y(t, \omega)=\pi f_{u}\left(t, x^{u_{*}}(t, \omega), u_{*}(t, \omega)\right)\left(u^{\prime}(t, \omega)-u^{*}(t, \omega)\right) .
\end{aligned}
$$

Then for $\theta=\beta_{0}\left(1+M^{+}\left\|u^{*}\right\|_{2}\right)+\left\|u^{*}\right\|_{2}$, we have 


$$
\theta^{-1} y(t, \omega)=\pi f_{u}\left(t, x^{u_{*}}(t, \omega), u_{*}(t, \omega)\right)\left[\theta^{-1}\left(u^{\prime}(t, \omega)-u^{*}(t, \omega)\right)\right],
$$

note that $\left\|\theta^{-1}\left(u^{\prime}-u^{*}\right)\right\|_{2} \leq 1$, so (15) holds for $\alpha=\theta^{-1}, \breve{Z}(. .)=$.0 .

Remark S. (Proof of (19)). Let $u$ be any given element in $U$, $\beta(t, \omega)=p(t, \omega) f_{u}^{*}(t, \omega)+q(t, \omega) \bar{\sigma}_{u}^{*}(t, \omega)$, and let $b \in(0,1)$ be an arbitrarily given Lebesgue point of $t \rightarrow \beta(t,).\left(u-u^{*}(t,).\right): J \rightarrow L_{2}\left(\Omega, \Phi, \mathbb{R}^{n}\right)$. Then, for any $\phi \in L_{\infty}\left(\Omega, \Phi_{b}, \mathbb{R}\right), \quad 0 \leq \phi \leq 1$,

$$
\left|\delta^{-1}\left[\int_{b}^{b+\delta} \phi(.) \beta(t, .)\left(u-u^{*}(t, .)\right) \mathrm{d} t-\delta \phi(.) \beta(b, .)\left(u-u^{*}(b, .)\right)\right]\right|_{2} \rightarrow 0,
$$

when $\delta \downarrow 0$. We have

$0 \geq E\left\langle p(s,),. q^{w}(s,).\right\rangle=E \int_{b}^{b+\delta}\left\{p(t,.) f_{u}^{*}(t,) w.(t,)+.q(t,.) \bar{\sigma}_{u}^{*}(t,) w.(t,).\right\} \mathrm{d} t$, when $w=\phi 1_{[b, b+\delta]}(t)\left(u-u^{*}(t,).\right)$, for $1>s>b+\delta$. From this and (70) it follows that

$$
E\left[\phi(.) p(b, .) f_{u}^{*}(b, .)\left(u-u^{*}(b, .)\right)+\phi(.) q(b, .) \bar{\sigma}_{u}^{*}(b, .)\left(u-u^{*}(b, .)\right)\right] \leq 0 .
$$

Because $\phi$ was arbitrary, (19) follows for $t=b$, i.e. for a.e. $t$.

Remark T. Let $\left(\Omega^{\prime}, \Phi^{\prime}, \mu^{\prime}\right)$ be a finite measure space. If $h(x, \omega): X \times \Omega^{\prime} \rightarrow X^{\prime} \quad\left(X, X^{\prime}\right.$ Euclidean spaces) is continuous in $x$ and $\Phi^{\prime}$ measurable in $\omega, \sup _{x}|h(x, \omega)| \leq \alpha(\omega), \quad \alpha(.) \in L_{2}\left(\Omega^{\prime}, \Phi^{\prime}, \mu^{\prime}, \mathbb{R}\right)$, and $x_{n}(\omega) \rightarrow x(\omega)$ in $\mu^{\prime}$-measure, $\left(x_{n}(\omega)\right.$ and $x(\omega) \quad \Phi^{\prime}$-measurable), then $h\left(x_{n}(\omega), \omega\right) \rightarrow h(x(\omega), \omega)$ in $L_{2}\left(\Omega, \Phi^{\prime}, \mu^{\prime}, X^{\prime}\right)$. This result, which is a special case of Krasnoselskii's theorem (see p. 20 in [9]), can be proved as follows. By contradiction, assume for some $\varepsilon>0$ and for some subsequence $n_{j}$ that $\left|h\left(x_{n_{j}}(\omega), \omega\right)-h(x(\omega), \omega)\right|_{2}>\varepsilon$ for all $j$. A subsequence $x_{n_{j_{k}}}(\omega)=: x^{k}(\omega)$ converges $\mu^{\prime}$-a.e. to $x(\omega)$. Then, by continuity,

$h\left(x^{k}(\omega), \omega\right) \rightarrow h(x(\omega), \omega) \quad \mu^{\prime}$-a.e. and even in $L_{2}$, by Lebesgue's dominated convergence theorem. A contradiction has been obtained.

Remark U. (On L $\left.L^{2},|\cdot|_{2} \leq{ }_{2}|\cdot|\right)$

On $L^{2},|\cdot|_{2} \leq{ }_{2}|$.$| . To show this, for the component z^{j}($.$) of z($.$) , note that$

$$
\begin{aligned}
& E\left(z^{j}(.)^{2}\right)=E\left(\sum_{k=1}^{\infty} \Pi_{k} z^{j}(.)\right)^{2} \\
&=E\left\{\sum_{k}\left(\Pi_{k} z^{j}(.)\right)^{2}\right\}+E\left\{E\left[\sum_{i, k, i<k} 2 \Pi_{i} z^{j}(.) \Pi_{k} z^{j}(.) \mid \Phi_{1-1 / 2^{k-1}}\right]\right\} \\
&=E\left\{\sum_{k}\left(\Pi_{k} z^{j}(.)\right)^{2}\right\} \\
&+ \\
&+E\left\{\sum _ { i , k , i < k } 2 \Pi _ { i } z ^ { j } ( . ) E \left[E\left[z^{j}(.) \mid \Phi_{1-1 / 2^{k}}\right]\right.\right. \\
&\left.\left.\left.\left.\left.-E\left[z^{j}(.) \mid \Phi_{1-1 / 2^{k-1}}\right]\right] \mid \Phi_{1-1 / 2^{k-1}}\right) \mid \Phi_{1-1 / 2^{i}}\right]\right)\right\} \\
&=\sum_{k} E\left(\Pi_{k} z^{j}(.)\right)^{2},
\end{aligned}
$$

so

$$
\begin{aligned}
\left|z^{j}(.)\right|_{2} & \leq \sum_{k}\left|\Pi_{k} z^{j}(.)\right|_{2} \leq \sum_{k}\left(1 / 2^{k}\right) 2^{k}\left|\Pi_{k} z^{j}(.)\right|_{2} \\
& \leq \sum_{k}\left(1 / 2^{k}\right)_{2}\left|z^{j}(.)\right| \leq{ }_{2}\left|z^{j}(.)\right| .
\end{aligned}
$$

Remark V. (Proof of $(\mathrm{B}) \Rightarrow(\mathrm{A})$ with $Z_{*}=Z_{* *}=0$ in Remark 6) 
Let $T=1$. By Lemma A in Appendix, for some $K>0$, $\left|q^{w^{\prime \prime}, u_{*}}(t)\right|_{2} \leq K\left(E \int_{0}^{1}\left|w^{\prime \prime}(t, .)-u_{*}(t, .)\right|^{2} \mathrm{~d} t\right)^{1 / 2}$ when $w^{\prime \prime}, u_{*} \in U_{*}$. Let $\gamma:=1+n^{\prime} M^{+}\left|u^{*}\right|_{2} \geq \max _{u \in U_{*}}\left[\max \left\{\left|\pi f_{u}\left(t, x^{u}, u\right) u^{*}\right|_{2},\left|\pi \bar{\sigma}_{u}\left(t, x^{u}, u\right) u^{*}\right|_{2}\right\}\right]$, let $c \in[0,1)$ satisfy $(1-c)^{1 / 2} \leq\left(4 \beta_{0} M^{+}\left(n^{\prime}\right) K\right)^{-1}$, let $L_{c}^{n}=L_{2}^{\text {prog }}\left([1-c, 1] \times \Omega, \mathbb{R}^{n}\right)$ and let $|w|_{2}^{c}=\left(E \int_{c}^{1}|w|^{2} \mathrm{~d} t\right)^{1 / 2}$, $B_{c, \alpha}^{n}:=\left\{z \in L_{c}^{n}:|z|_{2}^{c}<\alpha\right\}$, $\breve{U}_{c}^{u_{*}}:=\left\{1_{[c, 1]} u+\left(1-1_{[c, 1]}\right) u_{*}, u \in U^{\prime},\left|u(., .)-u_{*}(., .)\right|_{2} \leq 8 \beta_{0} \gamma\right\}$. By (B) in Remark 5, for any $u(.,.) \in U_{*}$, any $v^{\prime}(.,),. w^{\prime}(.,.) \in B_{c, 4 \gamma}^{m^{*}}$, for some progressively measurable $w^{\prime \prime}(.,.) \in U^{\prime}, w^{\prime \prime}=0$ on $[0, c)$, for each $(t, \omega), t \in[c, 1]$,

$$
\begin{aligned}
& \left(v^{\prime}(t, \omega), w^{\prime}(t, \omega)\right) \\
& =\left(\pi f_{u}\left(t, x^{u}(t, \omega), u(t, \omega)\right) w^{\prime \prime}(t, \omega), \pi \bar{\sigma}_{u}\left(t, x^{u}(t, \omega), u(t, \omega)\right) w^{\prime \prime}(t, \omega)\right),
\end{aligned}
$$

where $\left|w^{\prime \prime}(t, \omega)\right| \leq \beta^{0}\left|\left(v^{\prime}(t, \omega), w^{\prime}(t, \omega)\right)\right|$, so $\left|w^{\prime \prime}(., .)\right|_{2}^{c} \leq 8 \beta^{0} \gamma$. Hence, by slight abuse of notation, we have that

$$
\begin{aligned}
\left(B_{c, 4 \gamma}^{m^{*}} \times B_{c, 4 \gamma}^{m^{*}}\right) \subset & \left\{1_{[c, 1]}\left(\pi f_{u}\left(t, x^{u}, u\right) w^{\prime \prime}, \pi \bar{\sigma}_{u}\left(t, x^{u}, u\right) w^{\prime \prime}\right): w^{\prime \prime} \in 1_{[c, 1]} U^{\prime},\right. \\
& \left.\left|1_{[c, 1]} w^{\prime \prime}(., .)\right|_{2}^{c} \leq 8 \beta^{0} \gamma\right\} .
\end{aligned}
$$

By the choice of $c$, for $w \in \breve{U}_{c}^{u_{*}}$,

$$
\begin{aligned}
& \left|1_{[c, 1]} f_{x}\left(t, x^{u}, u\right) q^{w, u_{*}}\right|_{2}^{c},\left|1_{[c, 1]} \bar{\sigma}_{x}^{j}\left(t, x^{u}, u\right) q^{w, u_{*}}\right|_{2}^{c} \\
& \leq M^{+}\left(\int_{c}^{1} E\left|q^{w, u_{*}}(\tau)\right|^{2} \mathrm{~d} \tau\right)^{1 / 2} \leq M^{+} K\left(\int_{c}^{1} E \int_{c}^{1}\left|w(t)-u_{*}(t)\right|^{2} \mathrm{~d} t \mathrm{~d} \tau\right)^{1 / 2} \\
& \leq M^{+} K(1-c)^{1 / 2}\left|w-u_{*}\right|_{2}^{c} \leq 8 M^{+} K \beta_{0} \gamma(1-c)^{1 / 2} \leq 2 \gamma / n^{\prime} .
\end{aligned}
$$

By Remark $\mathrm{W}$ and the previous inclusion, then $\left(B_{c, 2 \gamma}^{m^{*}} \times B_{c, 2 \gamma}^{m^{*}}\right) \subset \mathrm{cl}_{|\cdot|_{2}^{c}} \operatorname{co} D_{c}$, where

$$
\begin{aligned}
D_{c}:= & \left\{1 _ { [ c , 1 ] } \left(\pi f_{x}\left(t, x^{u}, u\right) q^{w, u_{*}}+\pi f_{u}\left(t, x^{u}, u\right) w,\right.\right. \\
& \left.\left.\pi \bar{\sigma}_{x}\left(t, x^{u}, u\right) q^{w, u_{*}}+\pi \bar{\sigma}_{u}\left(t, x^{u}, u\right) w\right): w \in \breve{U}_{c}^{u_{*}}\right\} .
\end{aligned}
$$

Now, by definition of $\gamma$, for $\delta^{+}>0$ small, $\left|\pi f_{u}\left(t, x^{u}, u\right) u_{*}\right|_{2}$ and $\left|\bar{\sigma}_{u}\left(t, x^{u}, u\right) u_{*}\right|_{2}$ are $\leq \gamma$ when $\left|u_{*}-u^{*}\right|_{2} \leq \delta^{+}$, so $B_{c, \gamma}^{m^{*}} \subset B_{c, 2 \gamma}^{m^{*}}-1_{[c, 1]} \pi f_{u}\left(t, x^{u}, u\right) u_{*}$ and $B_{c, \gamma}^{m^{*}} \subset B_{c, 2 \gamma}^{m^{*}}-1_{[c, 1]} \bar{\sigma}_{u}\left(t, x^{u}, u\right) u_{*}$, so $B_{c, \gamma}^{m^{*}} \times B_{c, \gamma}^{m^{*}} \subset \mathrm{cl}_{|\cdot|_{2}^{c}} \mathrm{co} D^{c}$, where

$$
\begin{aligned}
D^{c}:= & \left\{1 _ { [ c , 1 ] } \left(\pi f_{x}\left(t, x^{u}, u\right) q^{w, u_{*}}+\pi f_{u}\left(t, x^{u}, u\right)\left(w-u_{*}\right),\right.\right. \\
& \left.\left.\pi \bar{\sigma}_{x}\left(t, x^{u}, u\right) q^{w, u_{*}}+\pi \bar{\sigma}_{u}\left(t, x^{u}, u\right)\left(w-u_{*}\right)\right): w \in \breve{U}_{c}^{u_{*}}\right\} .
\end{aligned}
$$

Hence, $(\mathrm{B}) \Rightarrow(\mathrm{A})$ has been proved.

Let $\Phi_{t}$ be the natural filtration, augmented as before. Postulating now (A), by Ito's representation theorem, 
$L_{2}\left(\Omega, \Phi_{1}, \mathbb{R}^{m^{*}}\right) \subset\left\{v^{\prime}+\int_{c^{\prime}}^{1} w^{\prime} \mathrm{d} B_{t}: w^{\prime} \in L_{2}^{\text {prog }}\left(J \times \Omega, \mathbb{R}^{m^{*}}\right), v^{\prime} \in \mathbb{R}^{m^{*}}\right\}$, so by the linear interior mapping theorem, for some $|\cdot|_{2}$-ball $B_{2}^{\Omega}\left(0, \delta^{\prime \prime}\right)$ in $L_{2}\left(\Omega, \Phi_{1}, \mathbb{R}^{m^{*}}\right)$, $B_{2}^{\Omega}\left(0, \delta^{\prime \prime}\right) \subset\left\{c^{\prime} v^{\prime}+\int_{c^{\prime}}^{1} w^{\prime} \mathrm{d} B_{t}: w^{\prime} \in B_{c^{\prime}, \xi}^{m^{*}}, v^{\prime} \in B(0, \xi) \subset \mathbb{R}^{m^{*}}\right\}$. But then $B_{2}^{\Omega}\left(0, \delta^{\prime \prime}\right) \subset \mathrm{cl}_{\left.|\cdot|\right|_{2}} \operatorname{co}\left\{\int_{c^{\prime}}^{1}\left(a-z_{*}\right) \mathrm{d} t+\int_{c^{\prime}}^{1}\left(b-z_{* *}\right) \mathrm{d} B_{t}:(a, b) \in D_{u_{*}}\right\}$. $\subset \mathrm{cl}_{|\cdot|_{2}} \operatorname{co}\left\{\int_{0}^{1}\left(a-z_{*}\right) \mathrm{d} t+\int_{0}^{1}\left(b-z_{* *}\right) \mathrm{d} B_{t}:(a, b) \in \tilde{D}\right\}$

Remark W. Let $I$ be a set and let $X$ be a normed space. Let the maps $V$ and $W$ from $I$ into $X$ have the following properties. Given $\varepsilon>0, k \in(0,1), p \in X$, assume that $B(p, \varepsilon) \subset \operatorname{clcov}(I)$ and that $|v(i)-w(i)| \leq \varepsilon(1-k)$ for all $i$. Then $B(p, \varepsilon k) \subset \operatorname{clcow}(I)$.

For a proof see p. 327 in [11].

Remark X. (A nonzero continuous linear functional on $L^{2}$ vanishing on all $\left.L_{2}\left(\Omega, \Phi_{t}, \mathbb{R}^{m^{*}}\right), t<T\right)$

Let $T=1, \Phi_{t}$ the natural filtration corresponding to some given $B_{t}$. For simplicity, assume $m^{*}=1$. Choose a $v^{*} \in L^{2}$ such that $2^{j}\left|\Pi_{j} v^{*}\right|_{2}=j /(j+1)$. Then ${ }_{2}\left|v^{*}\right|=1$, so $v^{*}$ belongs to the ${ }_{2}|\cdot|$-boundary of the ${ }_{2}|\cdot|$-ball ${ }_{2} B(0,1)$ in $L^{2}$. Then for some nonzero continuous linear functional $\mu$ on $L^{2}$, $\left\langle{ }_{2} B(0,1), \mu\right\rangle \leq\left\langle v^{*}, \mu\right\rangle$. Let $t \in[0,1)$ and let $k$ be any given integer such that $t \leq 1-1 / 2^{k}$. If $\phi \in L_{2}\left(\Omega, \Phi_{1-1 / 2^{k}}, \mathbb{R}\right)$ and $|\phi|_{2} \leq 2^{-(k+1)} /(j+1)$, then $2^{j}\left|\Pi_{j}( \pm \phi)\right|_{2} \leq 2^{j} 2 \cdot 2^{-(k+1)} /(j+1) \leq 1 /(j+1) \quad$ for $\quad j \leq k \quad$ and $\quad 2^{j}\left|\Pi_{j}( \pm \phi)\right|_{2}=0$ for $j>k$, so $2^{j}\left|\Pi_{j}\left(v^{*} \pm \phi\right)\right|_{2} \leq 1$ for all $j\left(\Rightarrow v^{*} \pm \phi \in{ }_{2} B(0,1)\right)$. Then the inequality involving $\mu$ yields $\langle \pm \phi, \mu\rangle \leq 0$, i.e. $\mu$ vanishes on $L_{2}\left(\Omega, \Phi_{t}, \mathbb{R}\right)$, $t<1$. To show in detail that such a $v^{*}$ exists, let $v_{i}=1_{M_{i}}-1_{C_{M_{i}}}$,

$M_{i}=\left[B_{1-1 / 2^{i}}-B_{1-1 / 2^{i-1}} \in[0, \infty)\right]$. Then

$E\left[v_{i} \mid \Phi_{1-1 / 2^{i-1}}\right]=P\left[M_{i} \mid \Phi_{1-1 / 2^{i-1}}\right]-P\left[C M_{i} \mid \Phi_{1-1 / 2^{i-1}}\right]=0$, so for $j<i$,

$\Pi_{j} v_{i}=E\left[v_{i} \mid \Phi_{1-1 / 2^{j}}\right]-E\left[v_{i} \mid \Phi_{1-1 / 2^{j-1}}\right]=0$, and for $j>i$,

$\Pi_{j} v_{i}=E\left[v_{i} \mid \Phi_{1-1 / 2^{j}}\right]-E\left[v_{i} \mid \Phi_{1-1 / 2^{j-1}}\right]=v_{i}-v_{i}=0$. Letting

$v^{*}=\sum_{i=1}^{\infty}\left(1 / 2^{i}\right)(i /(i+1)) v_{i}$, we get

$2^{j} \Pi_{j} v^{*}=\Pi_{j}(j /(j+1)) v_{j}$

$=(j /(j+1)) E\left[1_{M_{j}}-1_{C_{M_{j}}} \mid \Phi_{1-1 / 2^{j}}\right]-E\left[1_{M_{j}}-1_{C_{M_{j}}} \mid \Phi_{1-1 / 2^{j-1}}\right]$,

$=(j /(j+1))\left(1_{M_{j}}-1_{C_{M_{j}}}\right)=(j /(j+1)) v_{j}$

so $2^{j}\left|\Pi_{j} v^{*}\right|_{2}=(j /(j+1))\left|v_{j}\right|_{2}=j /(j+1)$ and $\left|2^{j} \Pi_{j} v^{*}\right|_{\infty} \leq j /(j+1)$.

Note that $v^{*}$ belongs to the ${ }_{2}|\cdot|$-boundary of $B_{(2)}^{\infty}(0,1) \subset \mathrm{cl}_{(2)} L_{\infty}^{*}$ and the $\phi$ 's belong to $\mathrm{cl}_{(2)} L_{\infty}^{*}$, so the arguments above actually yield a nonzero ${ }_{2}|$.$| -$ continuous linear functional on $\mathrm{cl}_{(2)} L_{\infty}^{*}$, vanishing on all $L_{2}\left(\Omega, \Phi_{t}, \mathbb{R}\right), t<T$.

Remark Y. $\left(L_{2}^{1} \backslash \mathrm{cl}_{2} L_{\infty}^{\text {prog }}(J \times \Omega, R)\right.$ is nonempty).

On $I_{i}$ define $\psi=\sum_{i} 1_{I_{i}}(t) i 1_{\Omega_{i}}(\omega)$, where $\Omega_{i}$ are some arbitrarily chosen 
sets such that $\mathrm{E} 1_{\Omega_{i}}=1 / i^{2}, \Omega_{i} \in \Phi_{1-1 / 2^{i}}$. Then for all $t,|\psi(t, .)|_{2}=1$, so $\|\psi\|_{2}=1$ and $\psi \in L_{2}^{1}:=\left\{z \in L_{2}^{\text {prog }}(J \times \Omega, R) ;\|z\|_{2}<\infty\right\}$. Now

$\operatorname{essup}_{t} \psi(t, \omega) \geq i 1_{\Omega_{i}}(\omega)$ for all $i$, so $\left|\operatorname{essup}_{t} \psi(t, .)\right|_{\infty} \geq i$ for all $i$.

Now $\psi \notin \mathrm{Cl}_{2} L_{\infty}^{\text {prog }}(J \times \Omega, R)$. Let us show that. Assume by contradiction that $\|\alpha-\psi\|_{2} \leq 1 / 2$ for some $\alpha \in L_{\infty}^{\text {prog }}(J \times \Omega, R)$. Using the projection of $\alpha$ onto $\psi$ as in E. in the proof of Theorem 1, we have for some progressively measurable $\varphi \in[0,1]$, that $|\phi(t, \omega) \psi(t, \omega)-\psi(t, \omega)| \leq|\alpha(t, \omega)-\psi(t, \omega)|$, $|\phi(t, \omega) \psi(t, \omega)| \leq|\alpha(t, \omega)|, \operatorname{essup}_{t, \omega}|\alpha(t, \omega)|<\infty$.

For all $t,\left|1_{I_{i}}(t)(\phi(t, .)-1) i 1_{\Omega_{i}}(.)\right|_{2} \leq 1 / 2$. Then

$\left|1_{I_{i}}(t) i 1_{\Omega_{i}}(t, .)\right|_{2}-1 / 2 \leq\left|1_{I_{i}}(t) \phi(t, .) i 1_{\Omega_{i}}(t, .)\right|_{2}$, or

$\left|1_{I_{i}}(t) 1_{\Omega_{i}}(t, .)\right|_{2}-1 / 2 i \leq\left|1_{I_{i}}(t) \phi(t, .) 1_{\Omega_{i}}\right|_{2}$. Then $P\left(A_{i}^{t}\right) \geq 1 / 16 i^{2}$ where

$A_{i}^{t}=\left\{\omega: \phi^{2}(t, \omega) \geq 1 / 8\right\} \cap \Omega_{i}$, otherwise (using $\phi \leq 1$ )

$\left|1_{I_{i}}(t) \phi(t, .) 1_{\Omega_{i}}(t, .)\right|_{2} \leq\left|1_{I_{i}}(t) 1_{A_{i}^{t}}(t, .)\right|_{2}+\left|1_{I_{i}}(t)\left(1_{\Omega_{i}}(t, .)-1_{A_{i}^{t}}(t, \omega)\right) \phi(t, .)\right|_{2}$.

$\leq 1 / 4 i+1 / 8 i^{2}<1 / 2 i$

But then, for all $i$, $\operatorname{essup}_{t, \omega} \phi \psi \geq \operatorname{essup}_{t, \omega} 1_{I_{i}}(t) 1_{A_{i}^{t}}(t, \omega) \phi(t, \omega) \psi(t, \omega)$

$$
\begin{aligned}
& \geq \operatorname{essup}_{t, \omega} 1_{I_{i}}(t)(1 / 8) 1_{A_{t}^{t}}(t, \omega) \psi(t, \omega) \\
& \geq \operatorname{essup}_{t, \omega} 1_{I_{i}}(t)(1 / 8) 1_{A_{i}^{t}}(t, \omega) i \geq i / 8
\end{aligned}
$$

contradicting $\operatorname{essup}_{t, \omega} \phi \psi \leq \operatorname{essup}_{t, \omega}|\alpha(t, \omega)|<\infty$. 